Research Article

\title{
Combined Effects of Heat and Mass Transfer on MHD Free Convective Flow of Maxwell Fluid with Variable Temperature and Concentration
}

\author{
Muhammad Bilal Riaz $\mathbb{D}^{1},{ }^{1,2}$ Maryam Asgir, ${ }^{3,4}$ A. A. Zafar, ${ }^{3}$ and Shaowen Yao ${ }^{5}$ \\ ${ }^{1}$ Department of Mathematics, School of Science, University of Management and Technology, C-II Johar Town, \\ Lahore 54770, Pakistan \\ ${ }^{2}$ Institute for Groundwater Studies (IGS), University of the Free State Bloemfotain, South Africa \\ ${ }^{3}$ Department of Mathematics, Government College University Lahore, Lahore,54000, Pakistan \\ ${ }^{4}$ Department of Mathematics, Riphah International University, QIE Township, Lahore 54000, Pakistan \\ ${ }^{5}$ School of Mathematics and Information Science, Henan Polytechnic University, Jiaozuo, China \\ Correspondence should be addressed to Shaowen Yao; yaoshaowen@hpu.edu.cn
}

Received 18 November 2020; Revised 15 March 2021; Accepted 26 March 2021; Published 14 April 2021

Academic Editor: Fateh Mebarek-Oudina

Copyright (C) 2021 Muhammad Bilal Riaz et al. This is an open access article distributed under the Creative Commons Attribution License, which permits unrestricted use, distribution, and reproduction in any medium, provided the original work is properly cited.

\begin{abstract}
Heat and mass transfer combined effects on MHD natural convection for a viscoelastic fluid flow are investigated. The dynamics of the fluid are controlled by the motion of the plate with arbitrary velocity along with varying temperature and mass diffusion. The non-dimensional forms of the governing equations of the model are developed along with generalized boundary conditions and the resulting forms are solved by the classical integral (Laplace) transform technique/method and closed-form solutions are developed. Obtained generalized results are very important due to their vast applications in the field of engineering and applied sciences; few of them are highlighted here as limiting cases. Moreover, parametric analysis of system parameters $P_{r}, S, K_{c}, G_{T}, G_{c}, M, S_{c}, \lambda$ is done via graphical simulations.
\end{abstract}

\section{Introduction}

In science and in many engineering applications such as in condensation, evaporation, and chemical process, many transport processes are influenced by the combined action of the buoyancy forces from both heat and mass diffusion. Heat and mass transfer combined effects are studied extensively due to their significant role in chemical processing equipment, oceanic circulation, emergency cooling system of advanced nuclear reactors, cooling process of plastic sheets, formation and dissipation of the fog, processing and drying the food, temperature distribution and moisture of agriculture fields, and production of polymer. In recent years, a lot of practical applications attracted many scientists and engineers to pay a considerable amount of focus to learn the heat and mass effects either analytically or numerically [1-4].In industrial and engineering processes, most fluids are nonNewtonian. Since the non-Newtonian fluids deal more complexities due to the rheological behavior than Newtonian fluids, distinct models were proposed. The influence of heat and mass transfer in the non-Newtonian fluid is an important subject from the theoretical as well as practical point of view due to its abundant applications in industry and engineering. Common examples include polymer extrusion, the emergency cooling system of nuclear reactors, food processing, thermal welding, to name a few. Convective flow is a selfsustained flow with the effect of the temperature gradient. In literature, different theories are made to see the occurrence of heat and mass transfer in convective flows of different fluids. Mebarek-Oudina et al. [5] investigated the natural convective heat transfer phenomenon of water-based hybrid nanofluid in a porous medium along with the magnetic field. Das et al. [6] 
considered the natural convective flow of the electrically conducting fluid past on vertical plate embedded in a permeable medium and explored the impacts of heat and mass transfer. The heat transfer phenomenon of Casson nanofluid flow is taken into account by Abo-Dahab et al. [7]. They analyzed the problem with the convective boundary conditions and discussed the influence of chemical reaction and heat source. Sajad et al.[8] studied the heat transfer and magnetic effect on hybrid nanofluid. Nazish et al. [9] explored the influence of heat and concentration/mass transform with the existence of fields developed by magnetic in the Maxwell fluid model. Ahmad [10] explored the heat transfer for the Maxwell fluid on the stretching plate with the slip boundary on the velocity. They explored the numerical solutions and showed the heat flux effect using the Nusselt number and the Prandtl number. A computational analysis is performed to study the effects of the transverse magnetic field at the unsteady Poiseuille-Rayleigh-Benard flow by Marzougui et al. [11]. The thermal properties' effects on the soil temperature are modeled and investigated numerically by Belatrache [12]. To have more insight about heat and mass transfer mechanisms in fluid flow and their applications, readers are referred to review references [13-16].On the other hand, many researchers paid a significant amount of attention to the study of MHD free convective flows due to its numerous applications in solar and stellar structure, radio propagation, MHD pumps, MHD bearings, aerodynamics, polymer technology, petroleum industry, crude oil purification, glass fiber drawing, etc. In light of these applications, many researchers such as Rajput [17], Gupta [18], and El Amin [19] studied the MHD flow of different fluids. They found the exact solution for velocity, concentration, and temperature by the Laplace transform method. Heat and mass transfer simultaneous effects on MHD flow of Maxwell fluid have been investigated by Nadeem et al. [20]. Recently, the study of the unsteady boundary layer heat transfer of Maxwell viscoelastic fluid was carried out by Zhao et al. [21]. Ahmad [22] studied MHD viscous, with constant density, electro-conducting fluid in the existence of the radiation, thermal diffusion, free convection, and mass transfer flow. These results motivated Chaudhry et al. [23] and they used classical integral transform to obtain the exact solutions of natural MHD convective flow past on an accelerated surface submerged in a permeable medium. Das [24] developed the closed-form solution of the unsteady
MHD natural convection flow on a moving vertical plate accompanied by mass transfer and thermal radiation. Carrying on, Das et al. [25] investigated the time-dependent MHD natural convection flow past a moving vertical plate dipped in a porous medium and studied the different aspects of heat and mass transfer. They discussed the problem with the uniform, oscillating, and impulsive motions of the plate besides considering the constant heat and mass diffusion and implemented the Laplace integral transform to develop the analytic solutions.Motivated by these investigations, the objective of this manuscript is to study the combined effect of heat and mass transfer on MHD Maxwell fluid. Laplace integral transformation is used to obtain the unique solution of temperature, velocity, and concentration under the impact of generalized boundary conditions on temperature, velocity, and concentration. The importance of the problem is highlighted by showing its impact/applications in the field of engineering and applied sciences. The paper is organized into six sections. After the introductory section in Section 2, the dimensionless governing equations are developed. In Section 3, Laplace integral transform is implemented to find the exact solution of the temperature, velocity, and concentration field. In Section 4, some applications in different fields are discussed as limiting cases to justify our results. In Section 5, the effect of physical parameters is analyzed graphically. The concluding observation is listed at the end.

\section{Problem Formulation}

We studied here the motion of the viscoelastic, incompressible, electronically conducting Maxwell fluid due to plate motion with arbitrary velocity $u_{0} f^{\prime}\left(t^{\prime}\right)$. The plate is along $x$-axis and $y$-axis is considered normal on the plate. In the first instance, at $t=0$ the plate and fluid are at temperature $T_{\infty}^{\prime}$ and concentration $C_{\infty}{ }^{\prime}$. With the time $t=0^{+}$, the plate starts to move in its own axis. Then, the level of temperature and concentration takes up to $T_{\infty}\{\hat{\prime}\}+T_{w}\{\hat{\prime}\} h^{\prime}\left(t^{\prime}\right)$ and $C_{\infty}\{\hat{\prime}\}+C_{w}\{\hat{\prime}\} g^{\prime}\left(t^{\prime}\right)$ where $f^{\prime}\left(t^{\prime}\right), h^{\prime}\left(t^{\prime}\right)$ and $g^{\prime}\left(t^{\prime}\right)$ are piecewise continuous functions that vanish at $t=0$. Details of different parameters are given in Table 1. Momentum, energy, and concentration equations are formed as follows: 
TABLE 1: Nomenclature.

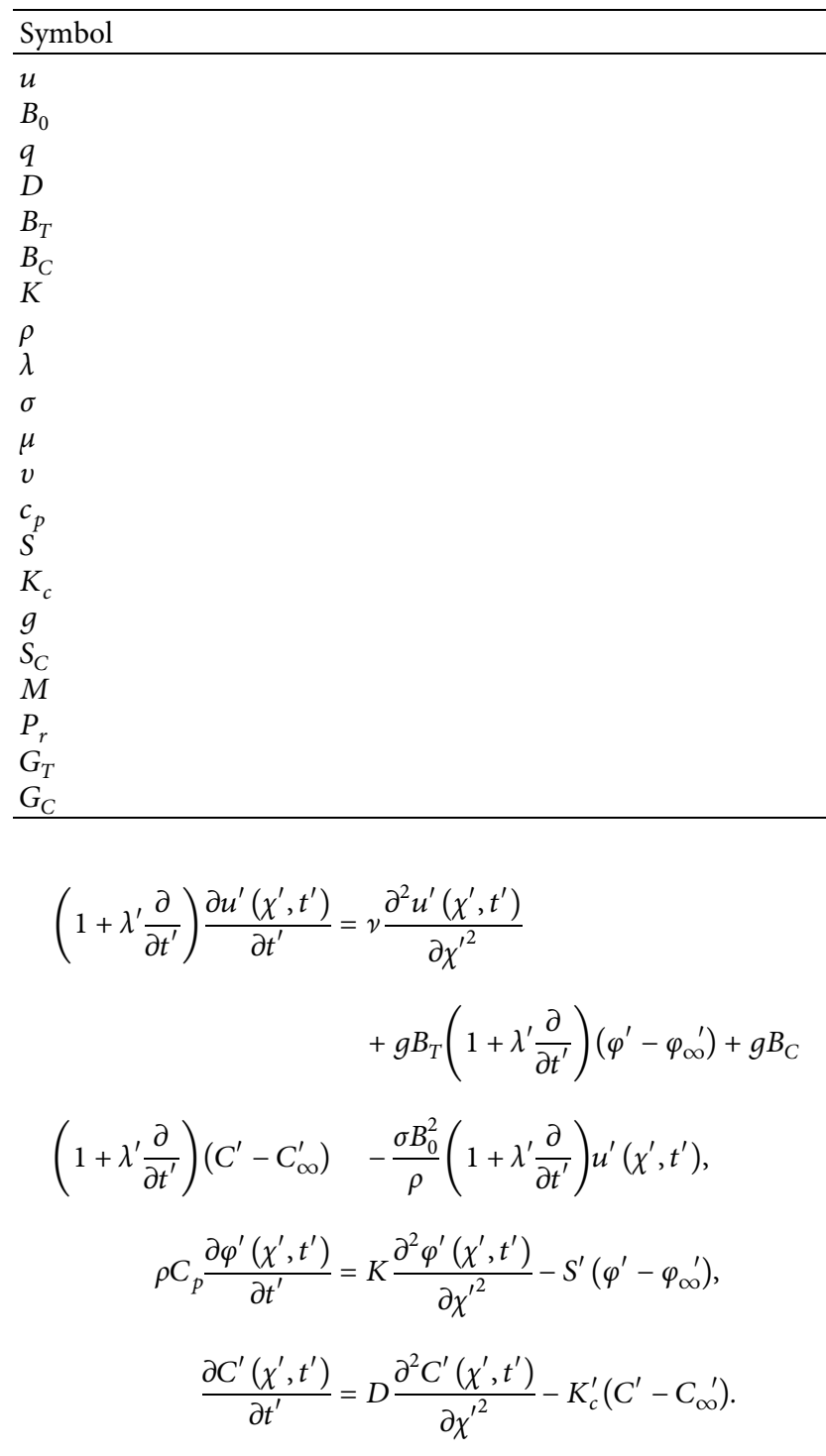

Quantity

Velocity of fluid

Magnetic field parameter Laplace transforms parameter Mass diffusivity

Thermal expansion parameter Concentration expansion coefficient

Thermal conductivity

Density of fluid

Relaxation time

Electric conductivity coefficient

Dynamic viscosity

Kinematic viscosity Specific heat

Heat source parameter

Chemical reaction coefficient

Gravitational acceleration

Schmidt number

Parameter due to magnetic field Prandtl number

Grashof number due to thermal effect Grashof number due to concentration

(1)

The imposed initial and boundary conditions are

$$
\begin{aligned}
t^{\prime} \leq 0, \quad u^{\prime}\left(\chi^{\prime}, t^{\prime}\right) & =0, \\
\varphi^{\prime}\left(\chi^{\prime}, t^{\prime}\right) & =\varphi_{\infty}^{\prime}, \\
C^{\prime}\left(\chi^{\prime}, t^{\prime}\right) & =C_{\infty}^{\prime}, \quad \chi^{\prime} \geq 0, \\
t^{\prime} \geq 0, \quad u^{\prime}\left(0, t^{\prime}\right) & =u_{0} f^{\prime}\left(t^{\prime}\right), \\
\varphi^{\prime}\left(0, t^{\prime}\right) & =\varphi_{\infty}^{\prime}+\varphi_{w}^{\prime} h^{\prime}\left(t^{\prime}\right), \\
C^{\prime}\left(0, t^{\prime}\right) & =C_{\infty}^{\prime}+C_{w}^{\prime} g^{\prime}\left(t^{\prime}\right), \\
u^{\prime}\left(\chi^{\prime}, t^{\prime}\right) & \longrightarrow 0, \\
\varphi^{\prime}\left(\chi^{\prime}, t^{\prime}\right) & \longrightarrow \varphi_{\infty}^{\prime}, \\
C^{\prime}\left(\chi^{\prime}, t^{\prime}\right) \longrightarrow C_{\infty}^{\prime}, \quad \chi^{\prime} & \longrightarrow \infty .
\end{aligned}
$$

For dimensionless problem, we use the following relations: 


$$
\begin{aligned}
& t=\frac{u_{0}^{2}}{v} t^{\prime}, \\
& y=\frac{u_{0}}{v} \chi^{\prime}, \\
& S_{c}=\frac{v}{D}, \\
& K_{c}=\frac{v}{u_{0}^{2}} K_{c}^{\prime}, \\
& \lambda=\frac{u_{0}^{2}}{v} \lambda^{\prime}, \\
& u^{\prime}=u_{0} u, \\
& C=\frac{C^{\prime}-C_{\infty}^{\prime}}{C_{w}^{\prime}}, \\
& T=\frac{\varphi^{\prime}-\varphi_{\infty}^{\prime}}{\varphi_{w}^{\prime}}, \\
& S=\frac{\nu}{\rho C_{p} u_{0}^{2} S^{\prime}}, \\
& P_{r}=\frac{\mu C_{p}}{K}, \\
& K_{p}^{\prime}=\frac{u_{0}^{2}}{v^{2}} K_{p} G_{c}=\frac{g\left(B_{C} / \nu\right)}{u_{0}^{3}} C_{w}^{\prime}, \\
& G_{T}=\frac{g B_{T} \nu}{u_{0}^{3}} \varphi_{w}^{\prime}, \\
& M=\frac{\sigma B_{0}^{2} \nu}{\rho u_{0}^{2}} \\
& f=\frac{v}{u_{o}^{2}} f^{\prime}, \\
& g=\frac{v}{u_{o}^{2}} g^{\prime} \\
& h=\frac{v}{u_{o}^{2}} h^{\prime} .
\end{aligned}
$$

After non-dimensionalizing, the governing equations become

$$
\begin{aligned}
\left(1+\lambda \frac{\partial}{\partial t}\right) \frac{\partial u(y, t)}{\partial t}= & \frac{\partial^{2} u(y, t)}{\partial y^{2}}+G_{T}\left(1+\lambda \frac{\partial}{\partial t}\right) T(y, t) \\
& +G_{C}\left(1+\lambda \frac{\partial}{\partial t}\right) C(y, t) \\
& -M\left(1+\lambda \frac{\partial}{\partial t}\right) u(y, t), \\
\frac{\partial T(y, t)}{\partial t}= & \frac{1}{P_{r}} \frac{\partial^{2} T(y, t)}{\partial y^{2}}-S T(y, t), \\
\frac{\partial C(y, t)}{\partial t}= & \frac{1}{S_{c}} \frac{\partial^{2} C(y, t)}{\partial y^{2}}-K_{c} C(y, t) .
\end{aligned}
$$

along the following initial and boundary conditions:

$$
\begin{gathered}
u(y, 0)=0, T(y, 0)=0, C(y, 0)=0, \\
u(0, t)=f(t), T(0, t)=h(t), C(0, t)=g(t), \\
u(y, t) \longrightarrow 0, T(y, t) \longrightarrow 0, C(y, t) \longrightarrow 0, \quad \text { as } y \longrightarrow \infty .
\end{gathered}
$$

\section{Solution of the Problem}

3.1. Concentration. Transforming equation (7) after applying the Laplace integral transform and utilizing the corresponding initial condition, we get

$$
\frac{\partial^{2} \bar{C}(y, q)}{\partial y^{2}}-S_{c}\left(K_{c}+q\right) \bar{C}(y, q)=0,
$$

The above differential equation solution is

$$
\bar{C}(y, q)=C_{1} e^{-y \sqrt{S_{c}\left(K_{c}+q\right)}}+C_{2} e^{y \sqrt{S_{c}\left(K_{c}+q\right)}} .
$$

The solution of equation (11) with the transformed form of boundary conditions becomes

$$
\bar{C}(y, q)=G(q) e^{-y \sqrt{s_{c}\left(K_{c}+q\right)}} .
$$


Applying the Laplace inverse on equation (12) and using the $L^{-1}\{G(q)\}=g^{\prime}(t)$ with $g(0)=0$, convolution theorem and equation (A.22), the generalized solution for concentration is

$$
C(y, t)=\int_{0}^{t} g^{\prime}(t-s) \Phi\left(y, \sqrt{S_{c}}, s ; K_{c}\right) \mathrm{d} s,
$$

and $\Phi$ is specified in equation (A.23).

3.2. Temperature Distribution. Implementing the Laplace transform on equation (5) and using the concerned the initial condition, we get

$$
\frac{\partial^{2} \bar{T}(y, q)}{\partial y^{2}}-P_{r}(S+q) \bar{T}(y, q)=0 .
$$

The solution is

$$
\bar{T}(y, q)=C_{1} e^{-y \sqrt{P_{r}(S+q)}}+C_{2} e^{y \sqrt{P_{r}(S+q)}} .
$$

After implementing the boundary conditions, equation (15) becomes

$$
\bar{T}(y, q)=H(q) e^{-y \sqrt{P_{r}(S+q)}} .
$$

The Laplace inverse on equation (16) and using the $L^{-1}\{H(q)\}=h^{\prime}(t)$ with $h(0)=0$, convolution theorem and equation (A.24), the generalized solution for temperature obtained is

$$
T(y, t)=\int_{0}^{t} h^{\prime}(t-s) \Psi\left(y, \sqrt{P_{r}}, s ; S\right) \mathrm{d} s,
$$

where $\Psi$ is defined in equation (A.25).

3.3. Velocity. Employing the Laplace transform on equation (4) and using the corresponding initial condition on velocity form the following differential equation:

$$
\begin{aligned}
\frac{\partial^{2} \bar{u}(y, q)}{\partial y^{2}}- & ((1+\lambda q)(q+M)) \bar{u}(y, q) \\
& \left.=-G_{T}(1+\lambda q) \bar{T}(y, q)\right)-G_{c}(1+\lambda q) \bar{C}(y, q) .
\end{aligned}
$$

In order to solve equation (18), we use the value of $\bar{C}(y, q), \bar{T}(y, q)$ from equation (12) and equation (16), respectively. With boundary conditions use on velocity, the following solution is obtained:

$$
\begin{aligned}
\bar{u}(y, q)= & F(q) e^{-y \sqrt{(q+M)(1+\lambda q)}}+\frac{G_{T} H(q)(1+\lambda q)\left(e^{-y \sqrt{(q+M)(1+\lambda q)}}-e^{-y \sqrt{P_{r}(S+q)}}\right)}{\left(P_{r}(S+q)-(1+\lambda q)(q+M)\right)} \\
& +\frac{G_{c} G(q)(1+\lambda q)\left(e^{-y \sqrt{(q+M)(1+\lambda q)}}-e^{-y \sqrt{S_{c}\left(K_{c}+q\right)}}\right)}{\left(S_{c}\left(K_{c}+q\right)-(1+\lambda q)(q+M)\right)}
\end{aligned}
$$

Further simplification reduces equation (19):

$$
\begin{aligned}
\bar{u}(y, q)= & F(q) e^{-y \sqrt{\lambda\left(\left(q+\alpha_{1}\right)^{2}+\alpha_{3}^{2}\right)}} \\
& -\frac{G_{T} H(q)(1+\lambda q)\left(e^{-y \sqrt{\lambda\left[\left(q+\alpha_{1}\right)^{2}+\alpha_{3}^{2}\right]}}-e^{-y \sqrt{P_{r}(S+q)}}\right)}{\lambda\left(\left(q+\alpha_{4}\right)^{2}-\alpha_{6}^{2}\right)} \\
& -\frac{G_{c} G(q)(1+\lambda q)\left(e^{-y \sqrt{\lambda\left[\left(q+\alpha_{1}\right)^{2}+\alpha_{3}^{2}\right]}}-e^{-y \sqrt{S_{c}\left(K_{c}+q\right)}}\right)}{\lambda\left(\left(q+\alpha_{7}\right)^{2}-\alpha_{9}^{2}\right)},
\end{aligned}
$$

where $2 \alpha_{1}=(\lambda M+1 / \lambda), \alpha_{2}=(M / \lambda), \alpha_{3}^{2}=\alpha_{2}-\alpha_{1}^{2}, 2 \alpha_{4}=$ $\left(\lambda M+1-P_{r} / \lambda\right), \alpha_{5}=\left(M-P_{r} S / \lambda\right), \quad \alpha_{6}^{2}=\alpha_{4}^{2}-\alpha_{5}, 2 \alpha_{7}=$ $\left(\lambda M+1-S_{c} / \lambda\right), \alpha_{8}=\left(M-S_{c} K_{c} / \lambda\right), \alpha_{9}^{2}=\alpha_{7}^{2}-\alpha_{8}$.
Generalized expression for velocity field is acquired by employing the inverse Laplace transform on equation (20):

$$
u(y, t)=I_{1}+\frac{G_{T}}{\lambda} I_{2}-\frac{G_{T}}{\lambda} I_{3}+\frac{G_{c}}{\lambda} I_{4}-\frac{G_{c}}{\lambda} I_{5},
$$

where

$$
\begin{aligned}
I_{1} & =L^{-1}\left(F(q) e^{\left.-y \sqrt{\lambda\left(\left(q+\alpha_{1}\right)^{2}+\alpha_{3}^{2}\right)}\right)},\right. \\
& =L^{-1}\left(B_{1}(y, q)\right) * L^{-1}\left(B_{2}(q)\right), \\
& =B_{1}(y, t) * B_{2}(t),
\end{aligned}
$$

where $B_{1}(y, q)=\left(e^{-\sqrt{\lambda} y \sqrt{\left(\left(q+\alpha_{1}\right)^{2}+\alpha_{3}^{2}\right)}} / \sqrt{\left(\left(q+\alpha_{1}\right)^{2}+\alpha_{3}^{2}\right)}\right)$, $B_{2}(q)=F(q) \sqrt{\left(\left(q+\alpha_{1}\right)^{2}+\alpha_{3}^{2}\right)}$.

By using equation (A.20) and equation (A.21), expressions for the $B_{1}(y, t)$ and $B_{2}(t)$ are evaluated as follows: 


$$
\begin{gathered}
B_{1}(y, t)= \begin{cases}0, & 0<t<y \sqrt{\lambda}, \\
e^{-\alpha_{1} t} I_{0}\left(i \alpha_{3} \sqrt{t^{2}-\lambda y^{2}}\right), & t>y \sqrt{\lambda},\end{cases} \\
B_{2}(t)=\left(e^{-\alpha_{1} t}\left(\left(i \alpha_{3}\right) I_{1}\left(i \alpha_{3} t\right)+\delta(t)\right)\right)^{*}\left(f^{\prime}(t)+\alpha_{1} f(t)\right) \\
\quad+\left(\alpha_{3}^{2} e^{-\alpha_{1} t} I_{0}\left(i \alpha_{3} t\right)\right)^{*} f(t),
\end{gathered}
$$$$
I_{2}=L^{-1}\left(\frac{H(q)(1+\lambda q) e^{-y \sqrt{P_{r}(S+q)}}}{\left(\left(q+\alpha_{4}\right)^{2}-\alpha_{6}^{2}\right)}\right),
$$$$
=\left(h(t)+\lambda h^{\prime}(t)\right)^{*}\left(e^{-\alpha_{4} t} \cosh \left(\alpha_{6} t\right)\right.
$$$$
\left.+\frac{\left(S-\alpha_{4}\right)}{\alpha_{6}} e^{-\alpha_{4} t} \sinh \left(\alpha_{6} t\right)\right)
$$$$
{ }^{*} \operatorname{erfc}\left(\frac{y \sqrt{P_{r}}}{2 \sqrt{t}}\right) e^{-S t} \text {. }
$$$$
I_{3}=L^{-1}\left(\frac{H(q)(1+\lambda q) e^{-y \sqrt{\lambda\left(\left(q+\alpha_{1}\right)^{2}+\alpha_{3}^{2}\right)}}}{\left(\left(q+\alpha_{4}\right)^{2}-\alpha_{6}^{2}\right)}\right),
$$$$
=B_{1}(y, t)^{*} B_{3}(t) \text {, }
$$

where $B_{1}(y, t)$ is given in equation (23) and

$$
\begin{aligned}
B_{3}(t)= & \left(h^{\prime}(t)+\lambda H(t)^{*} h^{\prime}(t)\right) \\
& *\left(A^{*}+B^{*} e^{-\left(\alpha_{4}+\alpha_{6}\right) t}+C^{*} e^{-\left(\alpha_{4}-\alpha_{6}\right) t}\right) \\
& * e^{-\alpha_{1} t} I_{0}\left(i \alpha_{3} t\right),
\end{aligned}
$$

where

$$
\begin{aligned}
A^{*} & =\frac{\left(\alpha_{1}^{2}+\alpha_{3}^{2}\right)}{\alpha_{4}^{2}-\alpha_{6}^{2}}, \\
B^{*} & =\frac{2\left(\alpha_{4}-\alpha_{6}\right) A^{*}-\left(2 \alpha_{1}-\alpha_{4}-\alpha_{6}\right)}{2 \alpha_{6}}, \\
C^{*} & =1-A^{*}-B^{*} . \\
I_{4}= & L^{-1}\left(\frac{G(q)(1+\lambda q) e^{-y \sqrt{S_{c}\left(K_{c}+q\right)}}}{\left(\left(q+\alpha_{7}\right)^{2}-\alpha_{9}^{2}\right)}\right), \\
= & \left(g(t)+\lambda g^{\prime}(t)\right)^{*}\left(e^{-\alpha_{7} t} \cosh \left(\alpha_{9} t\right)\right. \\
& \left.+\frac{\left(K_{c}-\alpha_{7}\right)}{\alpha_{9}} e^{-\alpha_{7} t} \sinh \left(\alpha_{9} t\right)\right), \\
& * \operatorname{erfc}\left(\frac{y \sqrt{S_{c}}}{2 \sqrt{t}}\right) e^{-K_{c} t} .
\end{aligned}
$$

where $B_{1}(y, t)$ is given in equation (23) and

$$
\begin{aligned}
B_{4}(t)= & \left(g^{\prime}(t)+\lambda H(t)^{*} g^{\prime}(t)\right) \\
& *\left(C^{*}+D^{*} e^{-\left(\alpha_{7}+\alpha_{9}\right) t}+E^{*} e^{-\left(\alpha_{7}-\alpha_{9}\right) t}\right) \\
& { }^{*} e^{-\alpha_{1} t} I_{0}\left(i \alpha_{3} t\right), \\
D^{*}= & \frac{\left(\alpha_{1}^{2}+\alpha_{3}^{2}\right)}{\alpha_{7}^{2}-\alpha_{9}^{2}} \\
E^{*}= & \frac{2\left(\alpha_{7}-\alpha_{9}\right) A^{*}-\left(2 \alpha_{1}-\alpha_{7}-\alpha_{9}\right)}{2 \alpha_{9}}, \\
F^{*}= & 1-D^{*}-E^{*} .
\end{aligned}
$$

The above results are obtained for generalized time-dependent boundary conditions on velocity, concentration, and temperature. These results have many applications in engineering and applied science. Now, we will consider and discuss its few applications.

\section{Applications}

4.1. Application 1: $f(t)=H(t), g(t)=H(t), h(t)=H(t)$. This function value shows the motion of the fluid is because of the motion of an infinite plate in its plane with constant velocity. This function has importance in a lot of engineering problems such as signal waves, driving forces that act for a short time only, and impulsive forces acting for an instance such as a hammer blow.Substituting the value of $G(q)=$ $(1 / q)$ into equation (12) and applying the Laplace inverse, the expression for concentration is

$$
C(y, t)=\left(\delta(t)+K_{c} t\right)^{*} \operatorname{erfc}\left(\frac{y \sqrt{S_{c}}}{2 \sqrt{t}}\right) e^{-K_{c} t},
$$

where $\delta($.$) is known as Dirac delta function.$

Embedding the value of $H(q)=(1 / q)$ into equation (16) and taking Laplace inverse make the expression of temperature

$$
T(y, t)=(\delta(t)+S t)^{*} \operatorname{erfc}\left(\frac{y \sqrt{P_{r}}}{2 \sqrt{t}}\right) e^{-S t} .
$$

The equation of velocity 


$$
u(y, t)=I_{1}^{I}+\frac{G_{T}}{\lambda} I_{2}^{I}-\frac{G_{T}}{\lambda} I_{3}^{I}+\frac{G_{c}}{\lambda} I_{4}^{I}-\frac{G_{c}}{\lambda} I_{5}^{I},
$$

where

$$
I_{1}^{I}=B_{1}(y, t)^{*} B_{2}^{I}(t),
$$

$B_{2}^{I}(t)$ is obtained as

$$
\begin{aligned}
B_{2}^{I}(t)= & \left(e^{-\alpha_{1} t}\left(\left(i \alpha_{3}\right) I_{1}\left(i \alpha_{3} t\right)+\delta(t)\right)\right)^{*}\left(\delta(t)+\alpha_{1} H(t)\right) \\
& +\alpha_{3}^{2} e^{-\alpha_{1} t} I_{0}\left(i \alpha_{3} t\right)^{*} H(t),
\end{aligned}
$$

and for $B_{1}(y, t)$ (see equation (A.1)).

After substituting the value of $H(q)=(1 / q)$ into equation (25)

$$
\begin{aligned}
I_{2}^{I}= & (H(t)+\lambda \delta(t))\left(e^{-\alpha_{4} t} \cosh \left(\alpha_{6} t\right)\right. \\
& \left.+\frac{\left(S-\alpha_{4}\right)}{\alpha_{6}} e^{-\alpha_{4} t} \sinh \left(\alpha_{6} t\right)\right) \\
& * \operatorname{erfc}\left(\frac{y \sqrt{P_{r}}}{2 \sqrt{t}}\right) e^{-S t} .
\end{aligned}
$$

Equation (26) takes the form after employing the value of $H(q)=(1 / q)$

$$
I_{3}^{I}=B_{1}(y, t)^{*} B_{3}^{I}(t)
$$

where

$$
\begin{aligned}
B_{3}^{I}(t)= & \left(\delta(t)+\lambda H(t)^{*} \delta(t)\right) \\
& *\left(A^{*}+B^{*} e^{-\left(\alpha_{4}+\alpha_{6}\right) t}+C^{*} e^{-\left(\alpha_{4}-\alpha_{6}\right) t}\right) \\
& * e^{-\alpha_{1} t} I_{0}\left(i \alpha_{3} t\right),
\end{aligned}
$$

and for $B_{1}(y, t)$ (see equation (A.1)).

After substituting the value of $G(q)=(1 / q)$ into equation (29)

$$
\begin{aligned}
I_{4}^{I}= & (H(t)+\lambda \delta(t))\left(e^{-\alpha_{7} t} \cosh \left(\alpha_{9} t\right)+\frac{\left(K_{c}-\alpha_{7}\right)}{\alpha_{9}} e^{-\alpha_{7} t} \sinh \left(\alpha_{9} t\right)\right) \\
& * \operatorname{erfc}\left(\frac{y \sqrt{S_{c}}}{2 \sqrt{t}}\right) e^{-K_{c} t}
\end{aligned}
$$

Similarly, equation (30) after substituting the value of $G(q)=(1 / q)$

$$
I_{5}^{I}=B_{1}(y, t)^{*} B_{4}^{I}(t)
$$

and

$$
\begin{aligned}
B_{4}^{I}(t)= & \left(\delta(t)+\lambda H(t)^{*} \delta(t)\right) \\
& *\left(D^{*}+E^{*} e^{-\left(\alpha_{7}+\alpha_{9}\right) t}+E^{*} e^{-\left(\alpha_{7}-\alpha_{9}\right) t}\right) \\
& * e^{-\alpha_{1} t} I_{0}\left(i \alpha_{3} t\right),
\end{aligned}
$$

and for $B_{1}(y, t)$ (see equation (A.1).
Similar result for concentration was obtained by Nehad Ali Shah [26] (equation (35)). Thus, our result supports the result already present in literature.

4.2. Application 2: $f(t)=t, g(t)=t, h(t)=t$. The important concepts of engineering are based around linear functions. They are often used in engineering to explain data and evaluate the lines that best fit the given data sets. It has a lot of applications in engineering and it can be represented in a variety of ways. One of the particular interests is direct variation, which forms many engineering applications such as Hooke's law and Ohm's law. To learn about slope, engineers use linear functions to interpret and understand graphs that describe displacement, velocity, and acceleration. They use these functions to analyze data to learn how to design their engineering products more efficiently, reliably, and safely.For the choice of $F(q), G(q), H(q)$ equal to $\left(1 / q^{2}\right)$ in the appropriate equations and employing the Laplace inverse, the expression of $C(y, t), T(y, t), u(y, t)$, and then $I_{1}^{I I}, I_{2}^{I I}, I_{3}^{I I}, I_{4}^{I I}$ and $I_{5}^{I I}$ changes into, respectively,

$$
\begin{aligned}
& C(y, t)=\left(1+K_{c} t\right)^{*} \operatorname{erfc}\left(\frac{y \sqrt{S_{c}}}{2 \sqrt{t}}\right) e^{-K_{c} t}, \\
& T(y, t)=(1+S t)^{*} \operatorname{erfc}\left(\frac{y \sqrt{P_{r}}}{2 \sqrt{t}}\right) e^{-S t}, \\
& u(y, t)=I_{1}^{I I}+\frac{G_{T}}{\lambda} I_{2}^{I I}-\frac{G_{T}}{\lambda} I_{3}^{I I}+\frac{G_{c}}{\lambda} I_{4}^{I I}-\frac{G_{c}}{\lambda} I_{5}^{I I},
\end{aligned}
$$

where

$$
I_{1}^{I I}=B_{1}(y, t)^{*} B_{2}^{I I}(t)
$$

where $B_{1}(y, t)$ (see equation (A.1)) and $B_{2}^{I I}(t)$ (see equation (A.2)).

$$
\begin{aligned}
I_{2}^{I I}= & (t+\lambda)^{*}\left(e^{-\alpha_{4} t} \cosh \left(\alpha_{6} t\right)+\frac{\left(S-\alpha_{4}\right)}{\alpha_{6}} e^{-\alpha_{4} t} \sinh \left(\alpha_{6} t\right)\right) \\
& * \operatorname{erfc}\left(\frac{y \sqrt{P_{r}}}{2 \sqrt{t}}\right) e^{-S t} \\
I_{3}^{I I}= & B_{1}(y, t)^{*} B_{3}^{I I}(t)
\end{aligned}
$$

where $B_{1}(y, t)$ (see equation (A.1)) and $B_{3}^{I I}(t)$ (see equation (A.3)).

$$
\begin{aligned}
I_{4}^{I I}= & (t+\lambda)^{*}\left(e^{-\alpha_{7} t} \cosh \left(\alpha_{9} t\right)+\frac{\left(K_{c}-\alpha_{7}\right)}{\alpha_{9}} e^{-\alpha_{7} t} \sinh \left(\alpha_{9} t\right)\right) \\
& * \operatorname{erfc}\left(\frac{y \sqrt{S_{c}}}{2 \sqrt{t}}\right) e^{-K_{c} t}, \\
I_{5}^{I I}= & B_{1}(y, t)^{*} B_{4}^{I I}(t),
\end{aligned}
$$


where $B_{1}(y, t)$ (see equation (A.1)) and $B_{4}^{I I}(t)$ (see equation (A.4)).

4.3. Application 3: $f(t)=\sin t, g(t)=\sin t, h(t)=\sin t$. The choice of this function shows the fluid motion due to the oscillation of the plate. It has a lot of applications in physics such as wave motion, other oscillatory motions, and engineering. It is used to model the behavior that repeats. Trigonometric functions are used to calculate angles in many engineering problems. In civil and mechanical engineering, trigonometry is used to calculate torque and forces on objects, which help build bridges and girders. In the construction of bridges, we need to consider the forces which keep the bridges at their balance and trigonometry helps us to calculate the static force which keeps the bridges static. In engineering, trigonometry is used to decompose the forces into horizontal and vertical components that can be analyzed.The expression for concentration after putting the value of $G(q)=\left(1 / q^{2}+1\right)$ into equation (12) is

$$
C(y, t)=\left(\cos t+K_{c} \sin t\right)^{*} \operatorname{erfc}\left(\frac{y \sqrt{S_{c}}}{2 \sqrt{t}}\right) e^{-K_{c} t},
$$

the expression for temperature become after putting the value of $H(q)=\left(1 / q^{2}+1\right)$ into equation (16) is

$$
T(y, t)=(\cos t+S \sin t)^{*} \operatorname{erfc}\left(\frac{y \sqrt{P_{r}}}{2 \sqrt{t}}\right) e^{-S t},
$$

and velocity change after substitute the value of $F(q)=\left(1 / q^{2}+1\right)$ into equation (21) is

$$
u(y, t)=I_{1}^{I I I}+\frac{G_{T}}{\lambda} I_{2}^{I I I}-\frac{G_{T}}{\lambda} I_{3}^{I I I}+\frac{G_{c}}{\lambda} I_{4}^{I I I}-\frac{G_{c}}{\lambda} I_{5}^{I I I},
$$

where

$$
I_{1}^{I I I}=B_{1}(y, t)^{*} B_{2}^{I I I}(t),
$$

where $B_{1}(y, t)$ (see equation (A.1)) and $B_{2}^{I I I}(t)$ (see equation (A.5)).

$$
\begin{aligned}
I_{2}^{I I I}= & (\sin t+\lambda \cos t)^{*}\left(e^{-\alpha_{4} t} \cosh \left(\alpha_{6} t\right)+\frac{\left(S-\alpha_{4}\right)}{\alpha_{6}} e^{-\alpha_{4} t} \sinh \left(\alpha_{6} t\right)\right) \\
& * \operatorname{erfc}\left(\frac{y \sqrt{P_{r}}}{2 \sqrt{t}}\right) e^{-S t}, \\
I_{3}^{I I I}= & B_{1}(y, t)^{*} B_{3}^{I I I}(t),
\end{aligned}
$$

where $B_{1}(y, t)$ (see equation (A.1)) and $B_{3}^{I I I}(t)$ (see equation (A.6)).

$$
\begin{aligned}
I_{4}^{I I I}= & (\sin t+\lambda \cos t)^{*}\left(e^{-\alpha_{7} t} \cosh \left(\alpha_{9} t\right)+\frac{\left(K_{c}-\alpha_{7}\right)}{\alpha_{9}} e^{-\alpha_{7} t} \sinh \left(\alpha_{9} t\right)\right) \\
& { }^{*} \operatorname{erfc}\left(\frac{y \sqrt{S_{c}}}{2 \sqrt{t}}\right) e^{-K_{c} t}, \\
I_{5}^{I I I}= & B_{1}(y, t)^{*} B_{4}^{I I I}(t),
\end{aligned}
$$

where $B_{1}(y, t)$ (see equation (A.1)) and $B_{4}^{I I I}(t)$ (see equation (A.7)).

4.4. Application 4: $f(t)=e^{t}, g(t)=e^{t}, h(t)=e^{t}$. The exponent functions are used for real-world application as for calculating area, volume, determining growth or decay, and impacts of force. In engineering, it helps them to design, build, and improve the machinery, structure, and equipment. For example, in sound engineering, it is used to calculate sound waves. In basic engineering, it is used to compute the tensile strength, which finds out the amount of stress that a structure can withstand. In aeronautical engineering, it is used to predict how airplanes, rockets, and jets will perform during flight. To determine the kinetic and potential energy, pressure, heat, and airflow of waves behavior, it is very helpful. Nuclear power sources are one of the important things developed by nuclear engineers. They used the exponents to work with extremely small numbers to make the big things happen. Substituting the value of $G(q)=$ $(1 / q-1)$ into equation (12), the concentration equation after implementing the Laplace inverse becomes

$$
C(y, t)=\left(e^{t}+K_{c} e^{t}\right)^{*} \operatorname{erfc}\left(\frac{y \sqrt{S_{c}}}{2 \sqrt{t}}\right) e^{-K_{c} t},
$$

and equation of temperature distribution after putting the value of $H(q)=(1 / q-1)$ into equation (16) and applying Laplace inverse

$$
T(y, t)=\left(e^{t}+S e^{t}\right)^{*} \operatorname{erfc}\left(\frac{y \sqrt{P_{r}}}{2 \sqrt{t}}\right) e^{-S t} .
$$

The expression for velocity is

$$
u(y, t)=I_{1}^{I V}+\frac{G_{T}}{\lambda} I_{2}^{I V}-\frac{G_{T}}{\lambda} I_{3}^{I V}+\frac{G_{c}}{\lambda} I_{4}^{I V}-\frac{G_{c}}{\lambda} I_{5}^{I V},
$$

where

$$
I_{1}^{I V}=B_{1}(y, t)^{*} B_{2}^{I V}(t)
$$

and $B_{1}(y, t)$ (see equation (A.1)) and $B_{2}^{I V}(t)$ (see equation (A.8)). 


$$
\begin{aligned}
I_{2}^{I V}= & \left(e^{t}+\lambda e^{t}\right)^{*}\left(e^{-\alpha_{4} t} \cosh \left(\alpha_{6} t\right)+\frac{\left(S-\alpha_{4}\right)}{\alpha_{6}} e^{-\alpha_{4} t} \sinh \left(\alpha_{6} t\right)\right) \\
& * \operatorname{erfc}\left(\frac{y \sqrt{P_{r}}}{2 \sqrt{t}}\right) e^{-S t}, \\
I_{3}^{I V}= & B_{1}(y, t)^{*} B_{3}^{I V}(t),
\end{aligned}
$$

and $B_{1}(y, t)$ (see equation (A.1)) and $B_{3}^{I V}(t)$ (see equation (A.9)).

$$
\begin{aligned}
I_{4}^{I V}= & \left(e^{t}+\lambda e^{t}\right)^{*}\left(e^{-\alpha_{7} t} \cosh \left(\alpha_{9} t\right)+\frac{\left(K_{c}-\alpha_{7}\right)}{\alpha_{9}} e^{-\alpha_{7} t} \sinh \left(\alpha_{9} t\right)\right) \\
& * \operatorname{erfc}\left(\frac{y \sqrt{S_{c}}}{2 \sqrt{t}}\right) e^{-K_{c} t} .
\end{aligned}
$$

Similarly,

$$
I_{5}^{I V}=B_{1}(y, t)^{*} B_{4}^{I V}(t)
$$

and $B_{1}(y, t)$ (see equation (A.1)) and $B_{4}^{I V}(t)$ (see equation (A.10)).

4.5. Application 5: $f(t)=t e^{t}, g(t)=t e^{t}, h(t)=t e^{t}$. Inserting the $G(q)=\left(1 /(q-1)^{2}\right)$ into equation (12) and applying the Laplace inverse, we get

$$
C(y, t)=\left(e^{t}+\left(K_{c}+1\right) t e^{t}\right)^{*} \operatorname{erfc}\left(\frac{y \sqrt{S_{c}}}{2 \sqrt{t}}\right) e^{-K_{c} t},
$$

and insert the $H(q)=\left(1 /(q-1)^{2}\right)$ into equation (16) and take Laplace inverse:

$$
\begin{aligned}
& T(y, t)=\left(e^{t}+(S+1) t e^{t}\right)^{*} \operatorname{erfc}\left(\frac{y \sqrt{P_{r}}}{2 \sqrt{t}}\right) e^{-S t}, \\
& u(y, t)=I_{1}^{V}+\frac{G_{T}}{\lambda} I_{2}^{V}-\frac{G_{T}}{\lambda} I_{3}^{V}+\frac{G_{c}}{\lambda} I_{4}^{V}-\frac{G_{c}}{\lambda} I_{5}^{V} .
\end{aligned}
$$

The $I_{1}^{V}$ takes the form after embedding the $F(q)=(1 /$ $\left.(q-1)^{2}\right)$

$$
I_{1}^{V}=B_{1}(y, t)^{*} B_{2}^{V}(t),
$$

where $B_{1}(y, t)$ (see equation (A.1)) and $B_{2}^{V}(t)$ (see equation (A.11)).

$$
\begin{aligned}
I_{2}^{V}= & \left(t e^{t}+\lambda e^{t}(t+1)\right)^{*}\left(e^{-\alpha_{4} t} \cosh \left(\alpha_{6} t\right)+\frac{\left(S-\alpha_{4}\right)}{\alpha_{6}} e^{-\alpha_{4} t} \sinh \left(\alpha_{6} t\right)\right) \\
& { }^{*} \operatorname{erfc}\left(\frac{y \sqrt{P_{r}}}{2 \sqrt{t}}\right) e^{-S t},
\end{aligned}
$$$$
I_{3}^{V}=B_{1}(y, t)^{*} B_{3}^{V}(t),
$$

where $B_{1}(y, t)$ (see equation (A.1)) and $B_{3}^{V}(t)$ (see equation (A.12)).

$$
\begin{aligned}
I_{4}^{V}= & \left(t e^{t}+\lambda e^{t}(t+1)\right)^{*}\left(e^{-\alpha_{7} t} \cosh \left(\alpha_{9} t\right)+\frac{\left(K_{c}-\alpha_{7}\right)}{\alpha_{9}} e^{-\alpha_{7} t} \sinh \left(\alpha_{9} t\right)\right) \\
& * \operatorname{erfc}\left(\frac{y \sqrt{S_{c}}}{2 \sqrt{t}}\right) e^{-K_{c} t}, \\
I_{5}^{V}= & B_{1}(y, t)^{*} B_{4}^{V}(t),
\end{aligned}
$$

where $B_{1}(y, t)$ (see equation (A.1)) and $B_{4}^{V}(t)$ (see equation (A.13)).

4.6. Application 6: $f(t)=e^{t} \sin t, g(t)=e^{t} \sin t, h(t)=e^{t}$ sin $t$. The choice of the value of $G(q)=\left(1 /(q-1)^{2}+1\right), H$ $(q)=\left(1 /(q-1)^{2}+1\right), F(q)=\left(1 /(q-1)^{2}+1\right)$ makes the expression

$$
\begin{aligned}
C(y, t) & =\left(e^{t}\left(\cos t+\left(1+K_{c}\right) \sin t\right)\right)^{*} \operatorname{erfc}\left(\frac{y \sqrt{S_{c}}}{2 \sqrt{t}}\right) e^{-K_{c} t}, \\
T(y, t) & =\left(e^{t}(\cos t+(1+S) \sin t)\right)^{*} \operatorname{erfc}\left(\frac{y \sqrt{P_{r}}}{2 \sqrt{t}}\right) e^{-S t}, \\
u(y, t) & =I_{1}^{V I}+\frac{G_{T}}{\lambda} I_{2}^{V I}-\frac{G_{T}}{\lambda} I_{3}^{V I}+\frac{G_{c}}{\lambda} I_{4}^{V I}-\frac{G_{c}}{\lambda} I_{5}^{V I}, \\
I_{1}^{V I} & =B_{1}(y, t)^{*} B_{2}^{V I}(t),
\end{aligned}
$$

where $B_{1}(y, t)$ (see equation (A.1)) and $B_{2}^{V I}(t)$ (see equation (A.14))

$$
\begin{aligned}
I_{2}^{V I}= & \left(e^{t} \sin t(1+\lambda)+e^{t} \cos t\right) \\
& *\left(e^{-\alpha_{4} t} \cosh \left(\alpha_{6} t\right)+\frac{\left(S-\alpha_{4}\right)}{\alpha_{6}} e^{-\alpha_{4} t} \sinh \left(\alpha_{6} t\right)\right) \\
& * \operatorname{erfc}\left(\frac{y \sqrt{P_{r}}}{2 \sqrt{t}}\right) e^{-S t}, \\
I_{3}^{V I}= & B_{1}(y, t)^{*} B_{3}^{V I}(t),
\end{aligned}
$$

where $B_{1}(y, t)$ (see equation (A.1)) and $B_{3}^{V I}(t)$ (see equation (A.15)).

$$
\begin{aligned}
I_{4}^{V I}= & \left(e^{t} \sin t(1+\lambda)+e^{t} \cos t\right) \\
& *\left(e^{-\alpha_{7} t} \cosh \left(\alpha_{9} t\right)+\frac{\left(K_{c}-\alpha_{7}\right)}{\alpha_{9}} e^{-\alpha_{7} t} \sinh \left(\alpha_{9} t\right)\right) \\
& * \operatorname{erfc}\left(\frac{y \sqrt{S_{c}}}{2 \sqrt{t}}\right) e^{-K_{c} t} .
\end{aligned}
$$

Similarly,

$$
I_{5}^{V I}=B_{1}(y, t)^{*} B_{4}^{V I}(t),
$$

where $B_{1}(y, t)$ (see (A.1)) and $B_{4}^{V I}(t)$ (see equation (A.16)). 
4.7. Application 7: $f(t)=t \sin t, g(t)=t \sin t, h(t)=t \sin$ $t$. By putting the value of $G(q)=\left(2 q /\left(q^{2}+1\right)^{2}\right)$ into equation (12) and employing Laplace inverse,

$$
C(y, t)=\left(t \cos t+\left(K_{c} t+1\right) \sin t\right)^{*} \operatorname{erfc}\left(\frac{y \sqrt{S_{c}}}{2 \sqrt{t}}\right) e^{-K_{c} t}
$$

By putting the value of $H(q)=\left(2 q /\left(q^{2}+1\right)^{2}\right)$ into equation (16) and Laplace inverse gives the expression

$$
\begin{aligned}
& T(y, t)=(t \cos t+(S t+1) \sin t)^{*} \operatorname{erfc}\left(\frac{y \sqrt{P_{r}}}{2 \sqrt{t}}\right) e^{-S t}, \\
& u(y, t)=I_{1}^{V I I}+\frac{G_{T}}{\lambda} I_{2}^{V I I}-\frac{G_{T}}{\lambda} I_{3}^{V I I}+\frac{G_{c}}{\lambda} I_{4}^{V I I}-\frac{G_{c}}{\lambda} I_{5}^{V I I},
\end{aligned}
$$

where

$$
I_{1}^{V I I}=B_{1}(y, t)^{*} B_{2}^{V I I}(t),
$$

and $B_{1}(y, t)$ (see equation (A.1)) and $B_{2}^{V I I}(t)$ (see equation (A.17)).

$$
\begin{aligned}
I_{2}^{V I I}= & ((t+\lambda) \sin t+\lambda \cos t) \\
& *\left(e^{-\alpha_{4} t} \cosh \left(\alpha_{6} t\right)+\frac{\left(S-\alpha_{4}\right)}{\alpha_{6}} e^{-\alpha_{4} t} \sinh \left(\alpha_{6} t\right)\right) \\
& * \operatorname{erfc}\left(\frac{y \sqrt{P_{r}}}{2 \sqrt{t}}\right) e^{-S t}, \\
I_{3}^{V I I}= & B_{1}(y, t)^{*} B_{3}^{V I I}(t),
\end{aligned}
$$

where $B_{1}(y, t)$ (see equation $\left.(\mathrm{A} .1)\right)$ and $B_{3}^{V I I}(t)$ (see equation (A.18)).

$$
\begin{aligned}
I_{4}^{V I I}= & ((t+\lambda) \sin t+\lambda \cos t) \\
& *\left(e^{-\alpha_{7} t} \cosh \left(\alpha_{9} t\right)+\frac{\left(K_{c}-\alpha_{7}\right)}{\alpha_{9}} e^{-\alpha_{7} t} \sinh \left(\alpha_{9} t\right)\right) \\
& * \operatorname{erfc}\left(\frac{y \sqrt{S_{c}}}{2 \sqrt{t}}\right) e^{-K_{c} t}, \\
I_{5}^{V I I}= & B_{1}(y, t)^{*} B_{4}^{V I I}(t),
\end{aligned}
$$

and for $B_{1}(y, t)$ (see equation (A.1)) and $B_{4}^{V I I}(t)$ (see equation (A.19)).These are solutions for the choice of same function for $f(t), g(t)$, and $h(t)$ from the list of functions $H(t), t, \sin t, e^{t}, t e^{t}, t \sin t, \sin t e^{t}$. We can consider the problem with the different choice of function for $f(t), g(t), h(t)$, e.g., $f(t)=t, g(t)=e^{t}, h(t)=H(t)$ and find its solution. For the validation of results, if we take $\lambda=$ $0, G_{T}=1, S=0, h(t)=1-a e^{b t}, g(t)=1$ with choice of $f(t)=H(t) t^{\alpha},(\alpha>0)$ or $\sin t$, in our system of equations $(4)-(8)$, the results obtained are the same as the result obtained by Nehad Ali shah [27] (choosing the $\epsilon=0, N=$ $G_{C}$ in equation (9)).

\section{Results and Discussion}

The heat and mass transfer study of Maxwell fluid is discussed here. The solutions for dimensionless velocity, concentration, and temperature are assessed by the Laplace transform method. The application of these solutions in different fields of engineering sciences is also discussed. It brings to attention that these results are helpful to solve the complicated problems of engineering and applied science. The behavior of these solutions for velocity, concentration, and temperature profile is depicted graphically. The impacts of different pertinent parameters $\lambda, M, S_{c}, K_{c}, P_{r}, G_{T}, G_{C}, S$ on fluid flow are also deliberated using plots and their physical aspects described. To avoid repetition, only the most significant graphical representations regarding the effects of the concerned parameter will be included here.

The variation in the behavior of velocity and concentration with varying values of Schmidt number $S_{c}$ is illustrated inFigures 1-3, respectively. An increase in Schmidt number results in the decline in the thickness of the boundary layer of concentration. Since the Schmidt number is defined as the ratio between kinematic viscosity and mass diffusivity, it reduced the concentration as well as velocity profile. In reality, the increase occurring in momentum diffusivity causes a decline in the fluid velocity.

The deviation in temperature profile for varying values of Prandtl number Pr is demonstrated in Figures 4 and 5. It is depicted that the thermal boundary layer thickness decreases rapidly with the increase in the values of Pr. For a small value of $\mathrm{Pr}$, heat diffuses very quickly in comparison to the velocity. The reason is, the thermal boundary layer thickness in liquid metals is higher than the momentum boundary layer. Finally, $\operatorname{Pr}$ can be practiced to expand the percentage of cooling.

Similar effects can be seen for the heat absorption coefficient $S$ on temperature profile with different values of the function $g(t)$ at different time scales depicted in Figure 6 and 7. The thermal buoyancy forces decrease with the increase of $S$ which decreases the fluid temperature.

Impacts of magnetic parameter $M$ displayed in Figure 8 depict the velocity decline with the increase of magnetic parameter values. Physically, when magnetic force is applied to the velocity field, it generates the drag force known as the Lorentz force which opposes the motion of the fluid. Figure 9 shows the impact of Pr on velocity. The increase of $P_{r}$ results in a decrease in velocity. The velocity boundary layer gets thicker due to the low rate of thermal diffusion. Basically, in heat transfer problems, $P_{r}$ control the relative thickness momentum boundary layer.

In Figure 10, the study of the effects of $G_{T}$ on velocity describes the increase in behavior with increment in the values of $G_{T}$. Physically, the result of more induced fluid flows is due to a rise in buoyancy effects which is the result of the increase in $G_{T}$. The depiction of $G_{C}$ on velocity is portrayed in Figure 11. We can see the rise in velocity with the rise in the value of $G_{C}$. The natural convection and 

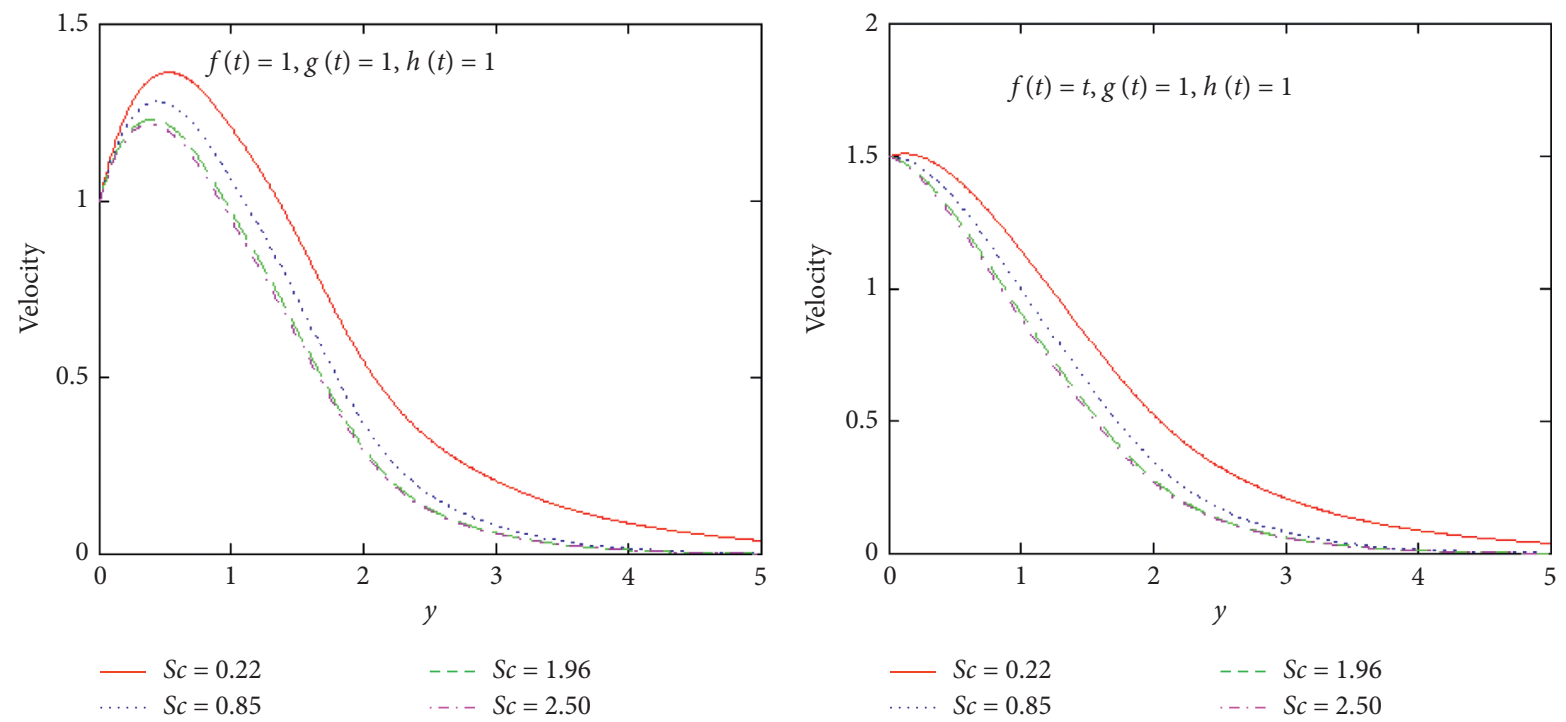

(a)

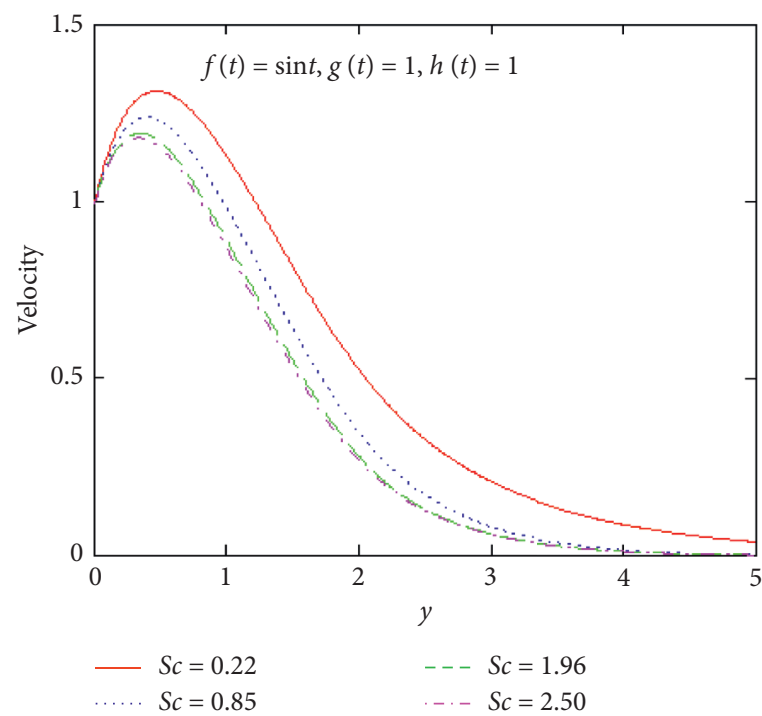

(c)

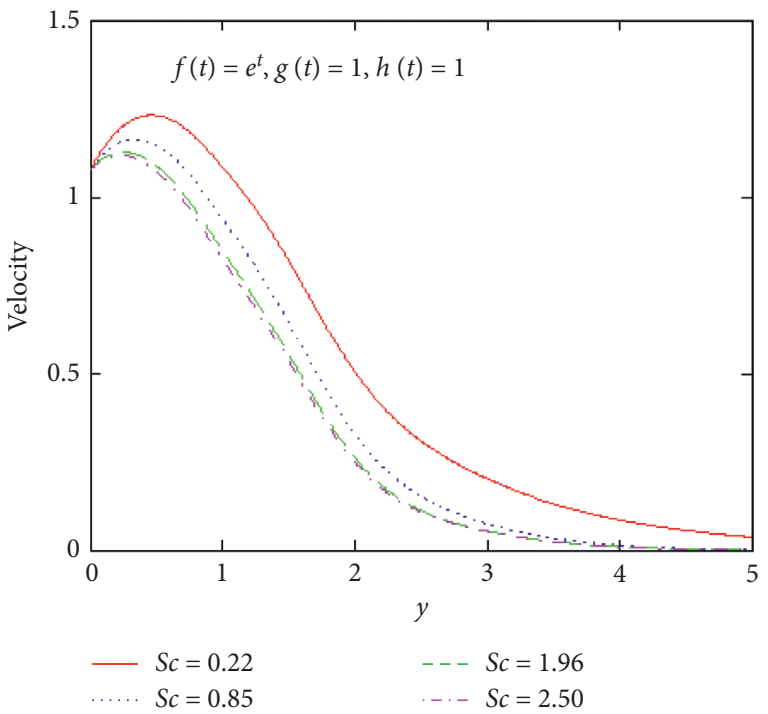

(d)
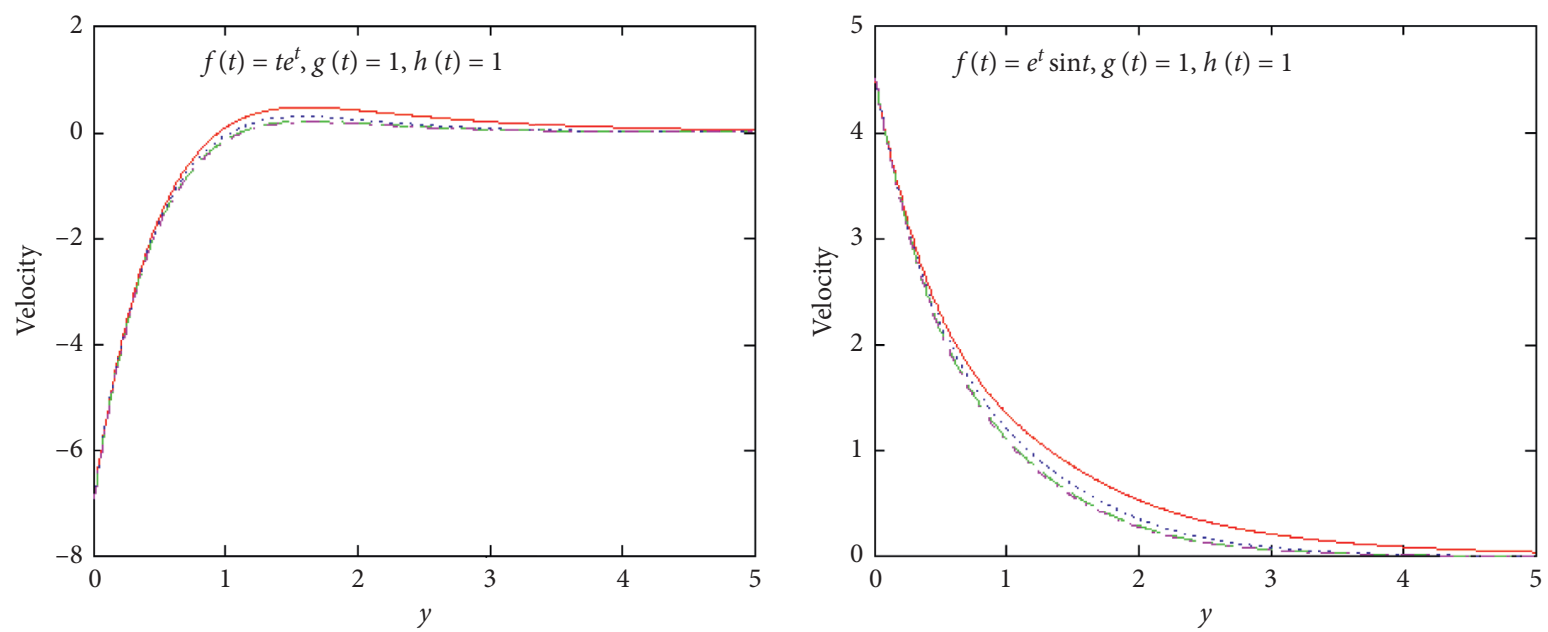

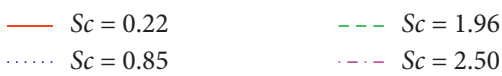

$\begin{aligned}-S c & =0.22 \\ \text { S. Sc } & =0.85\end{aligned}$

$---S c=1.96$

(e)

(f)

FIgURE 1: Continued. 


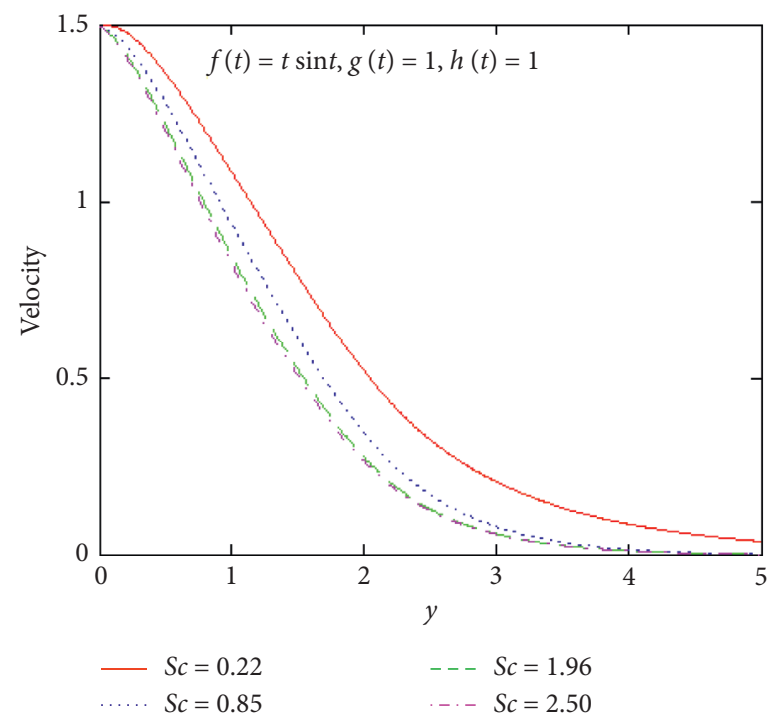

(g)

Figure 1: Profile of velocity for different values of $S_{c}$ and $M=2.0, \lambda=0.6, S=1.0 .5, K_{c}=0.5, G_{T}=5.0, G_{C}=2.0, P_{r}=0.71$.

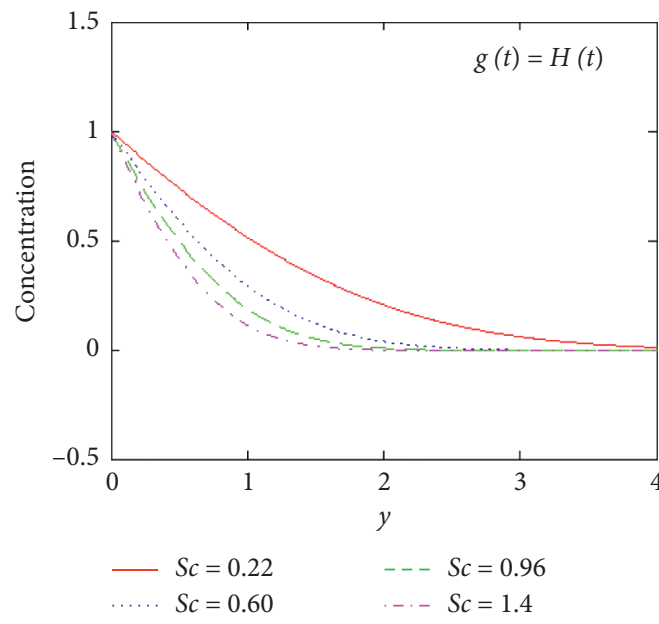

(a)

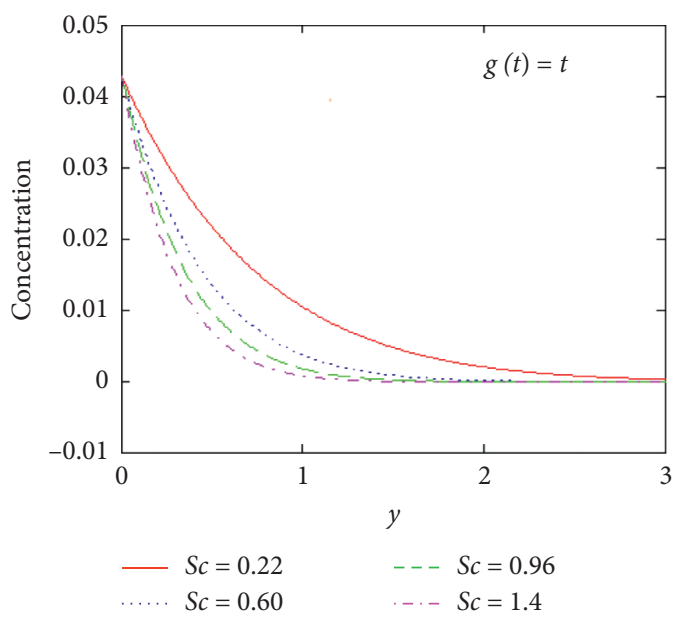

(c)

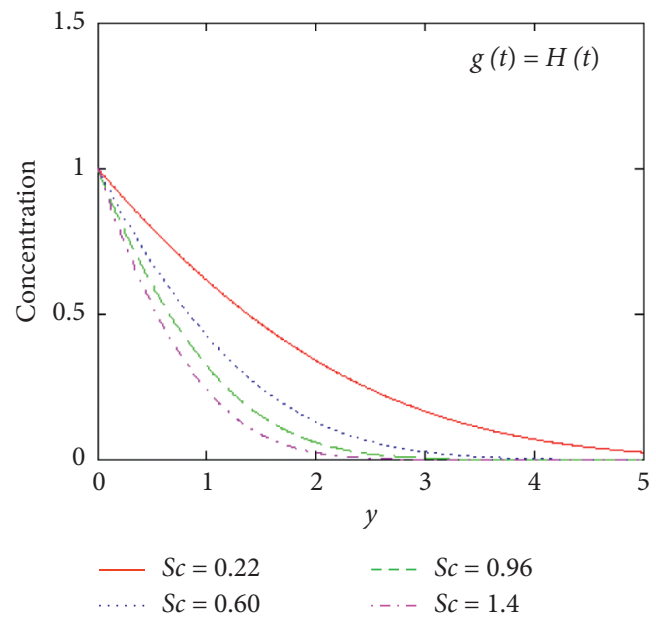

(b)

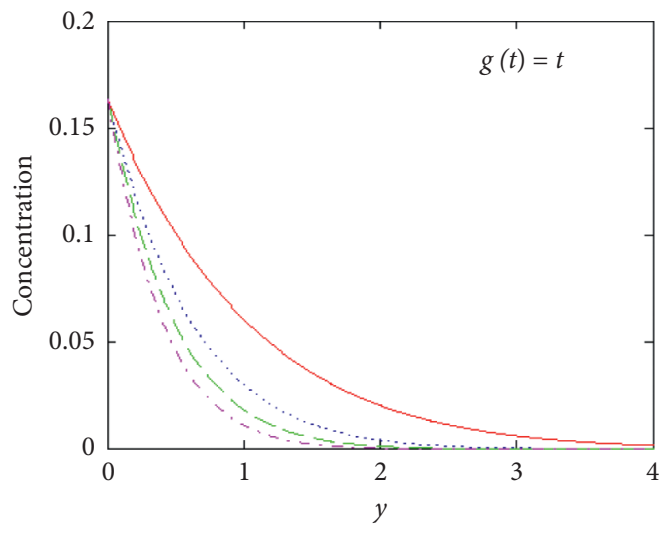

$\begin{aligned}-S c & =0.22 \quad--S c=0.96 \\ -\ldots S c & =0.60\end{aligned}$

(d)

FIgURE 2: Continued. 


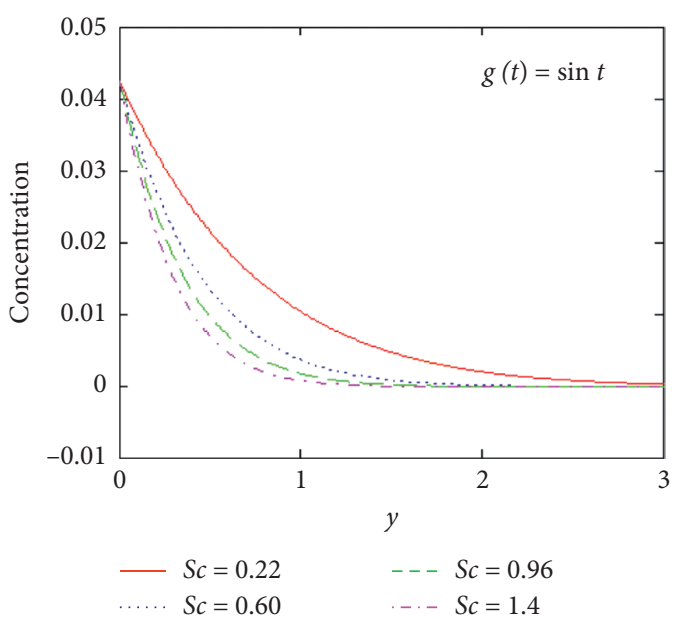

(e)

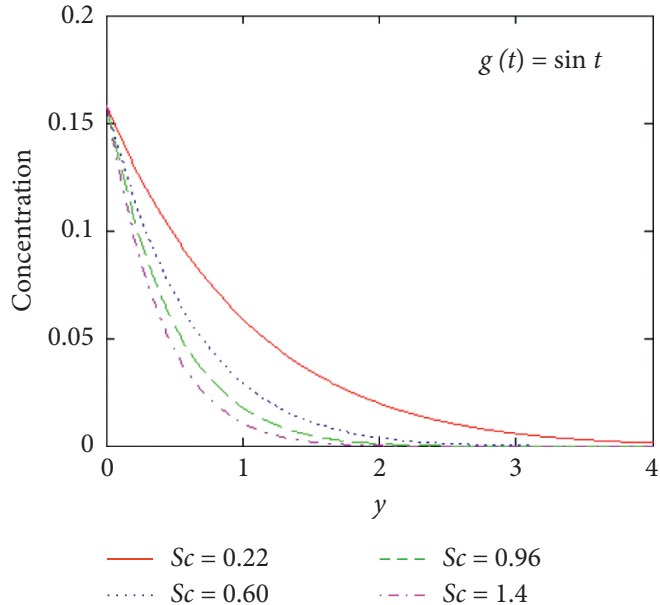

(f)

Figure 2: Profile of concentration for different values of $S_{c}$ and variation of time.

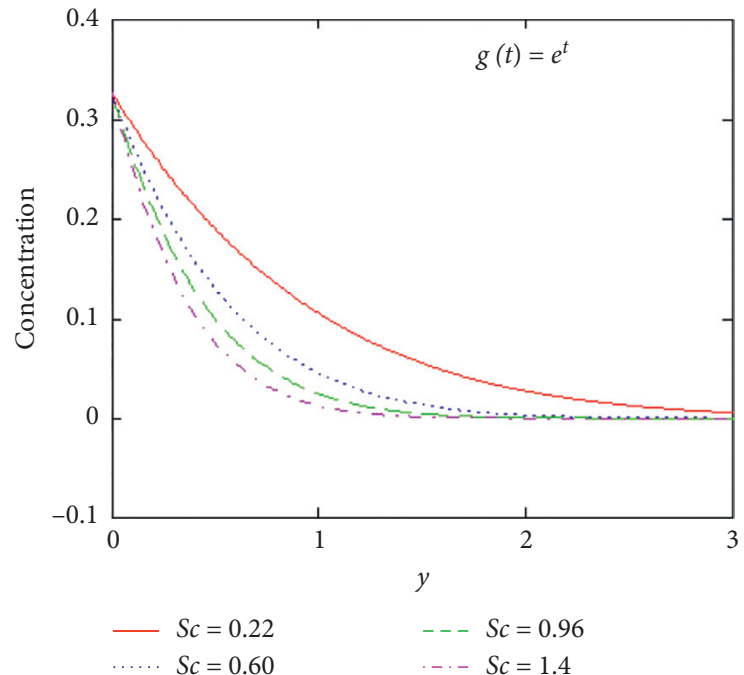

(a)

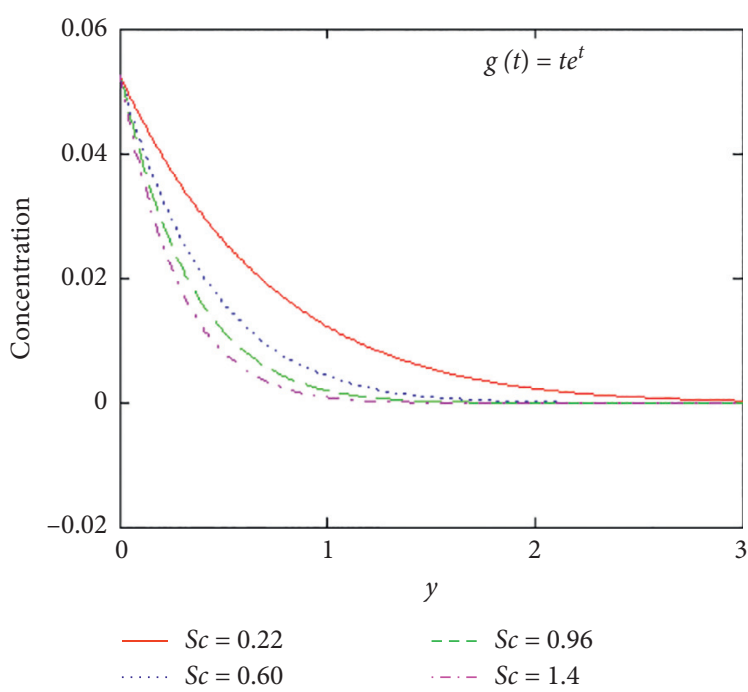

(c)

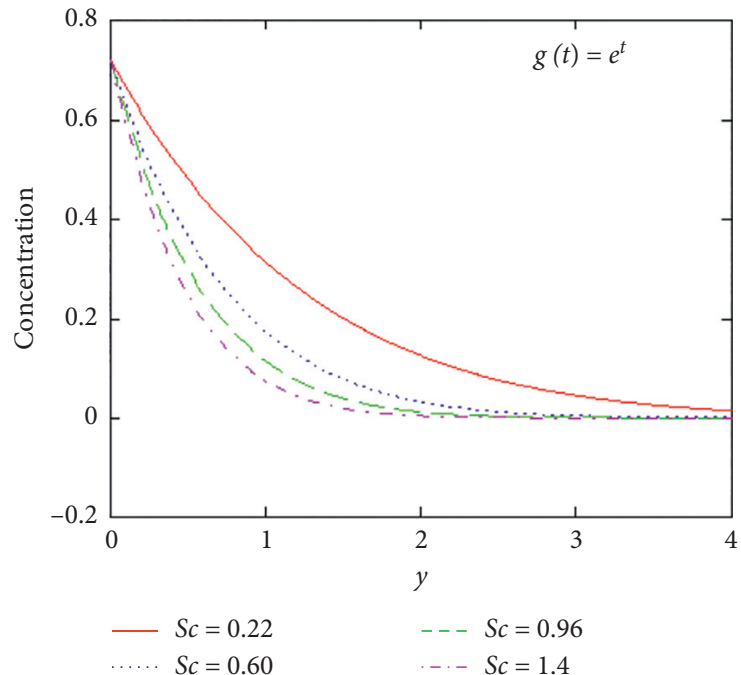

(b)

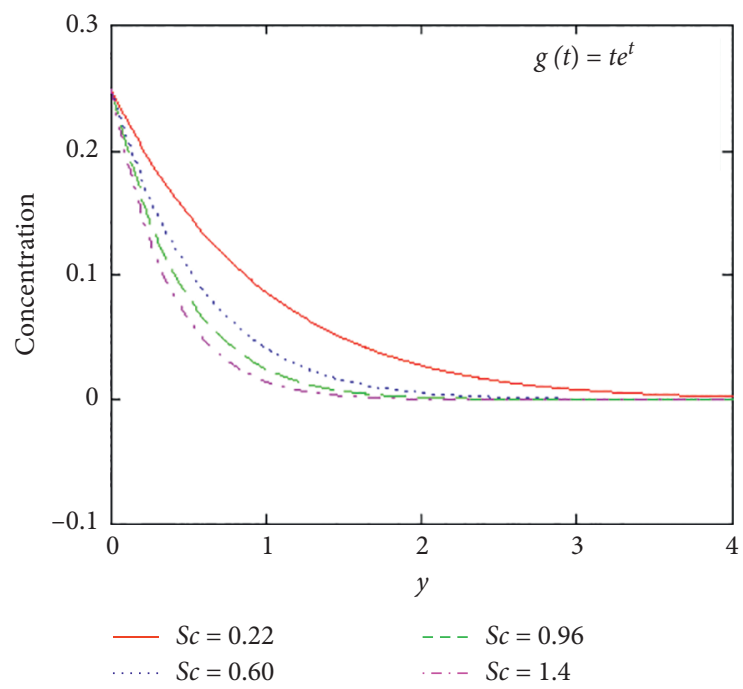

(d)

FIgure 3: Continued. 


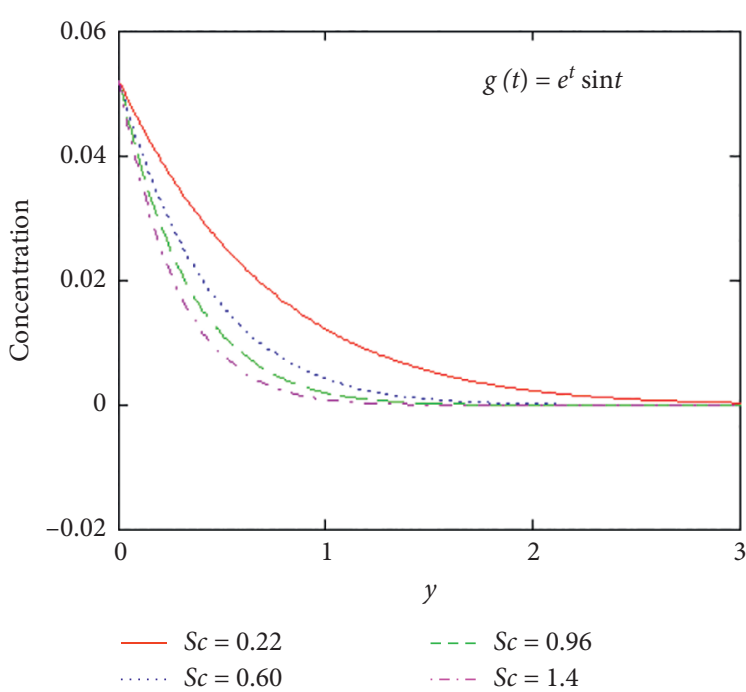

(e)

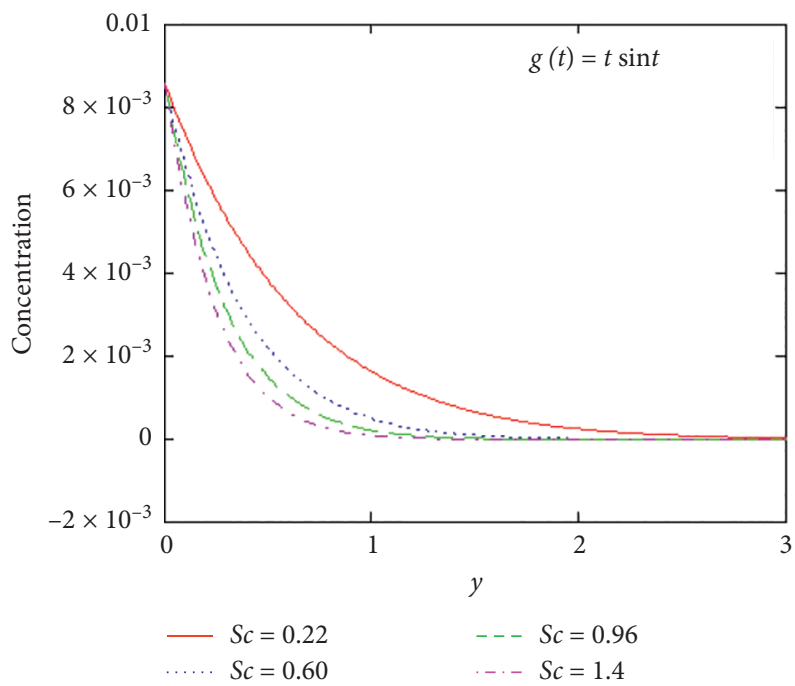

(g)

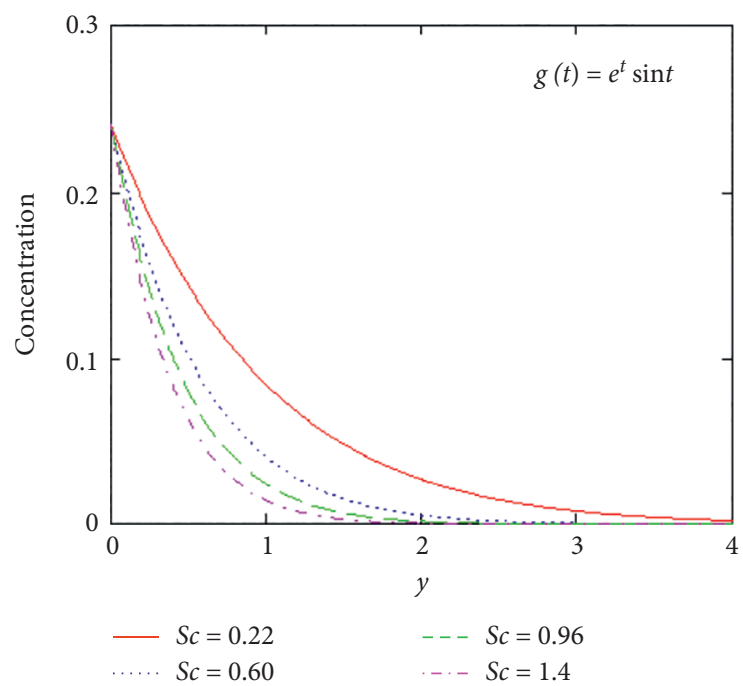

(f)

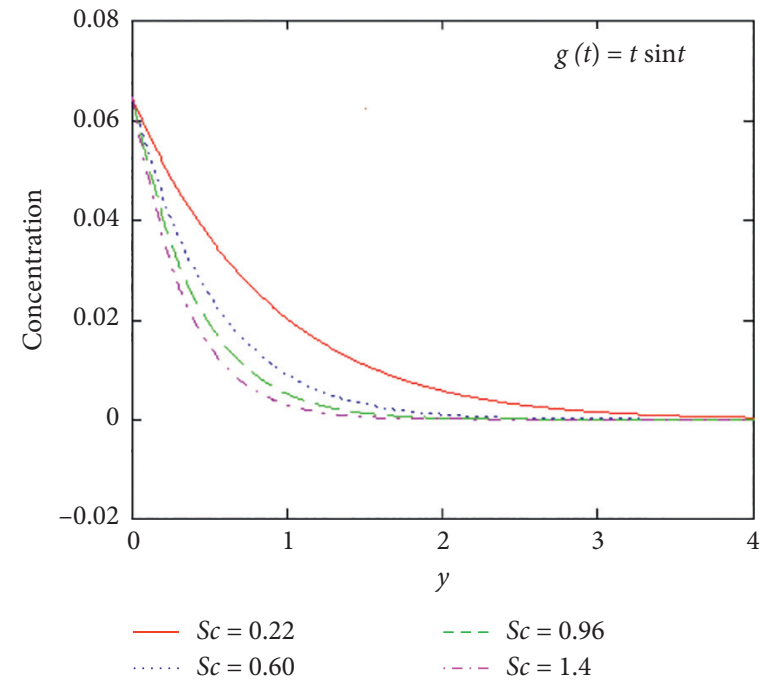

(h)

Figure 3: Profile of concentration for different values of $S_{c}$ and variation of time.

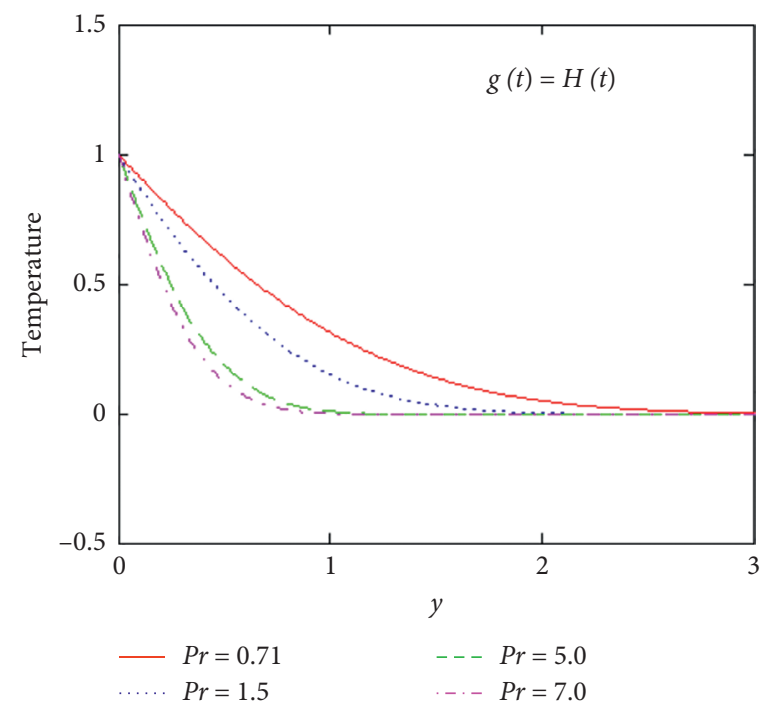

(a)

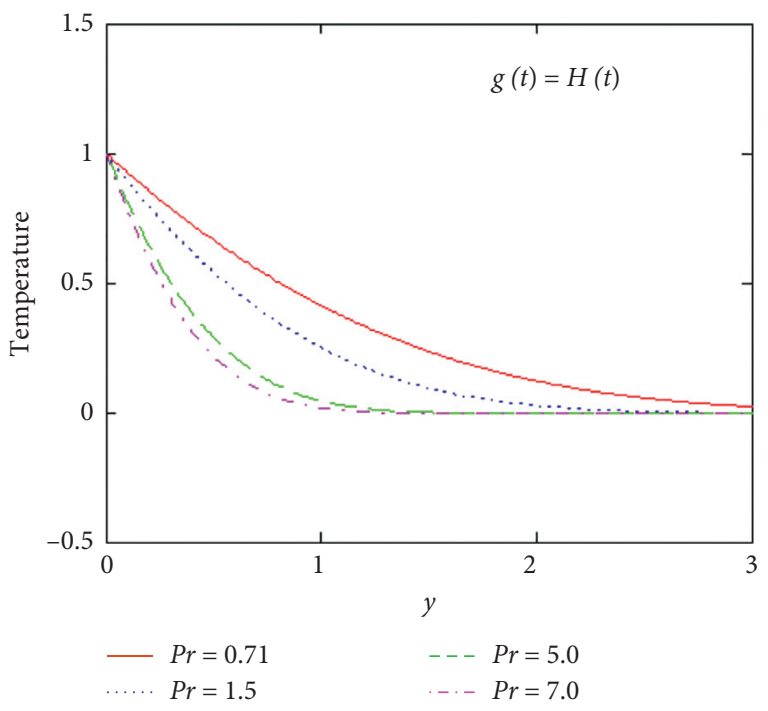

(b)

FIgUre 4: Continued. 


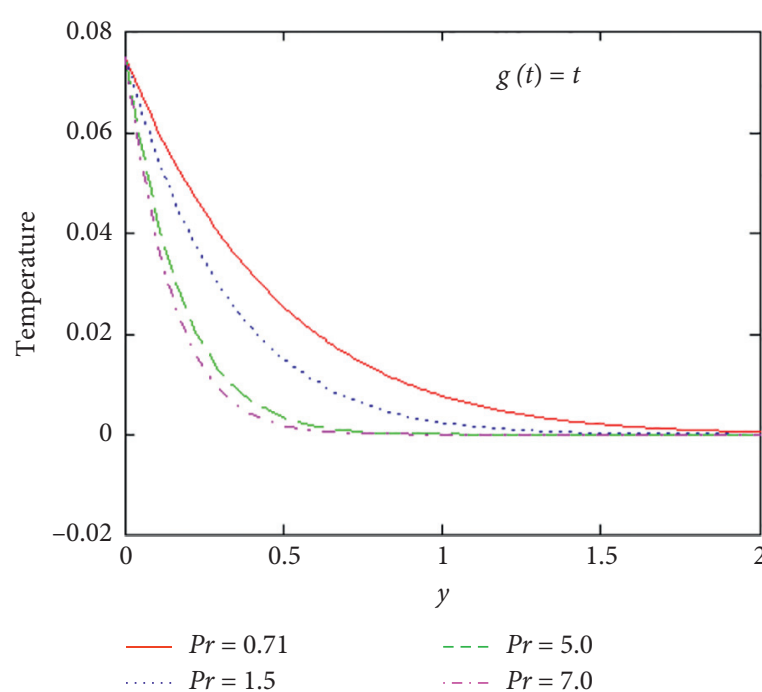

(c)

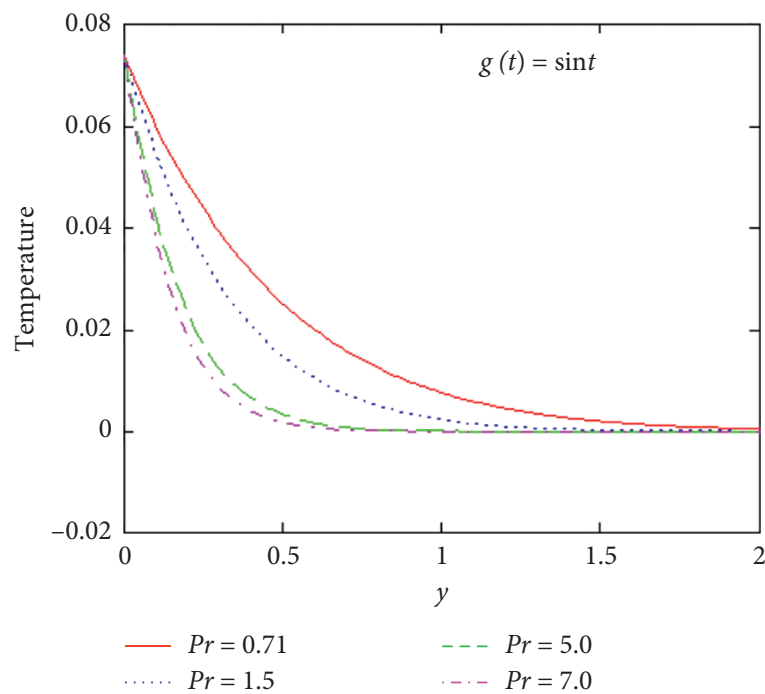

(e)

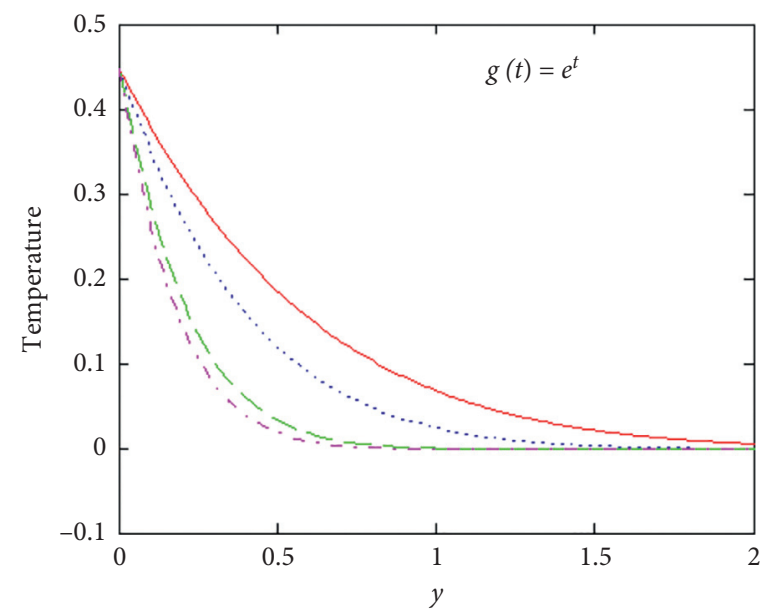

$\begin{aligned}-\operatorname{Pr} & =0.71 \\ \ldots \operatorname{Pr} & =1.5\end{aligned}$

(g)

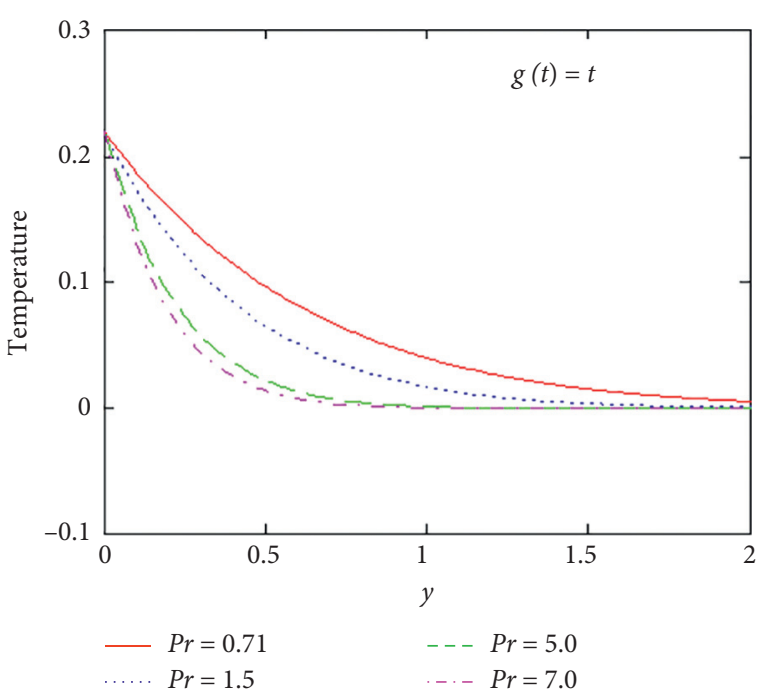

(d)

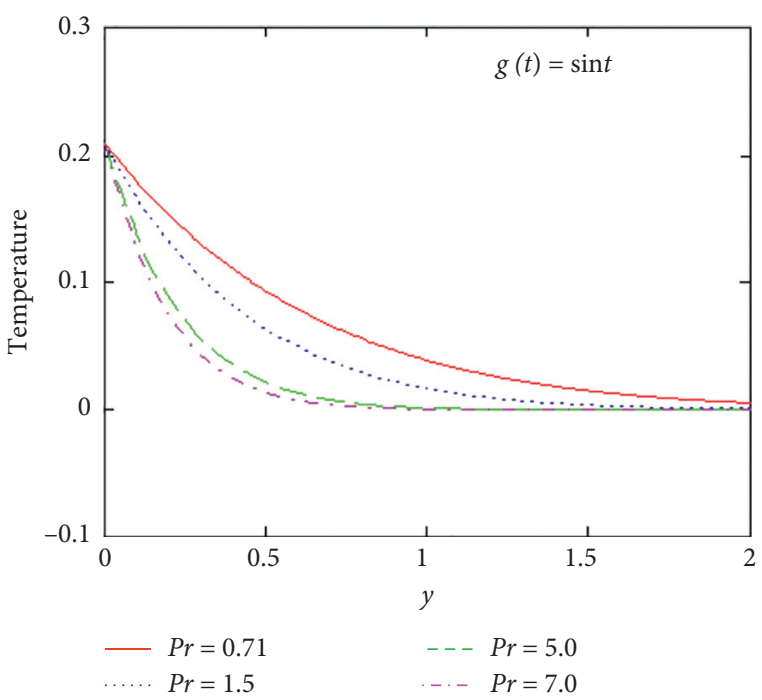

(f)
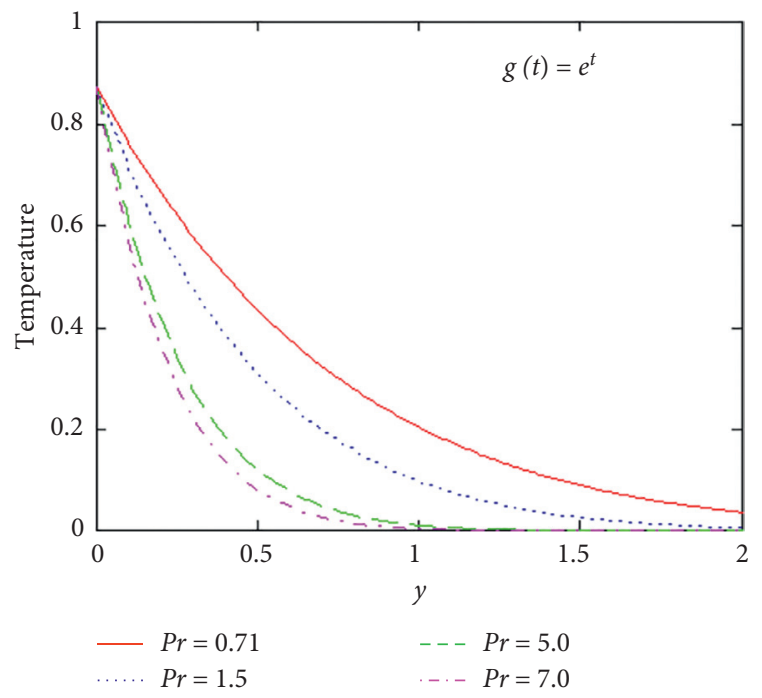

(h)

Figure 4: Profile of temperature for different values of $P_{r}$ and variation of time. 

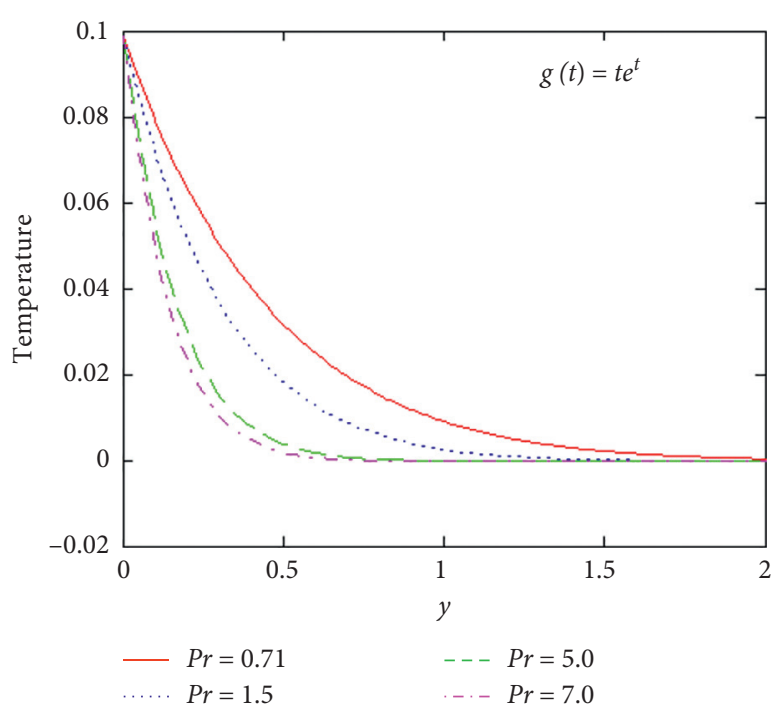

(a)
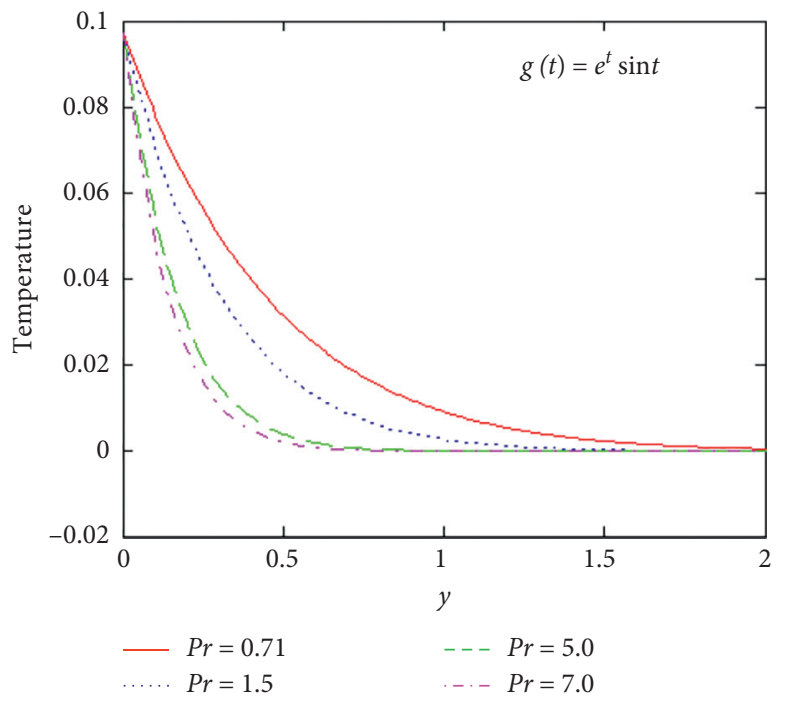

(c)

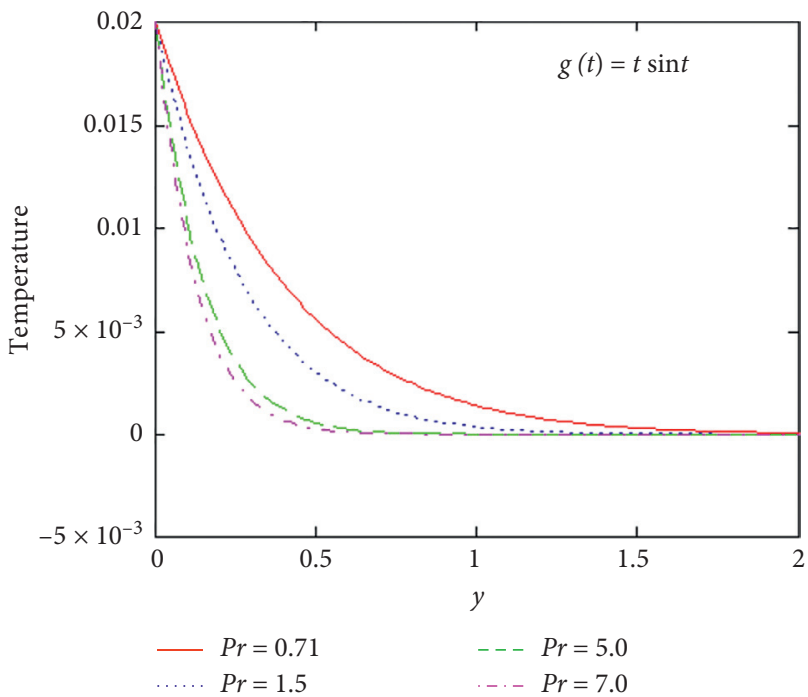

(e)
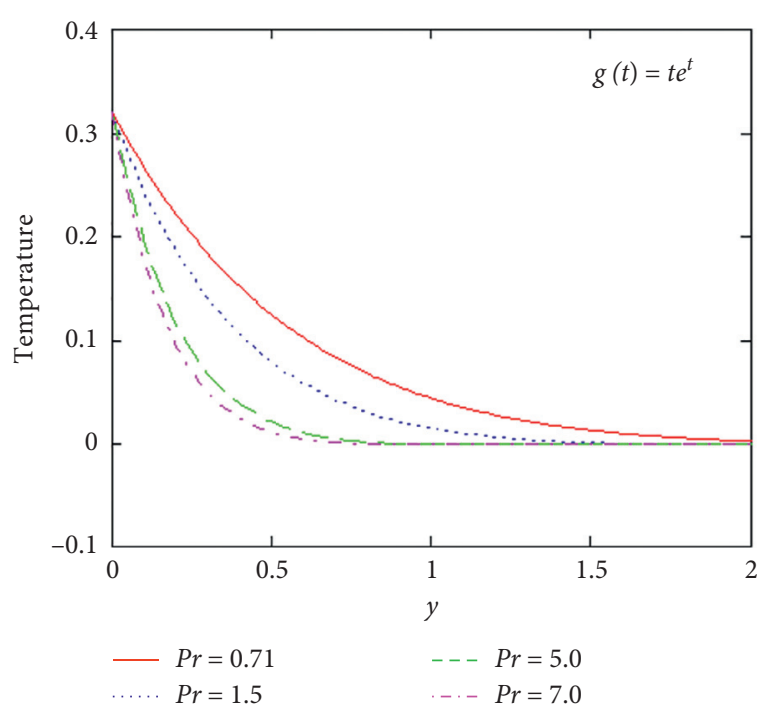

(b)
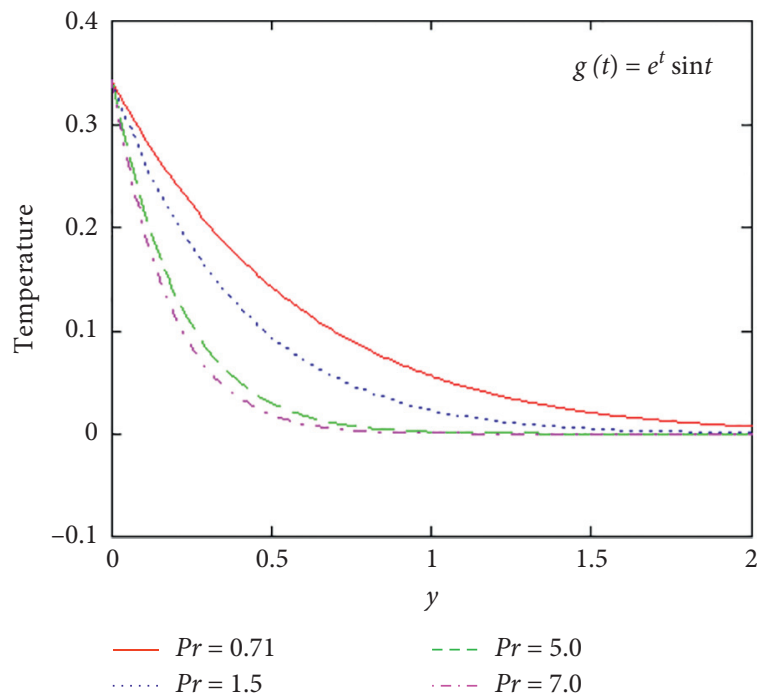

(d)

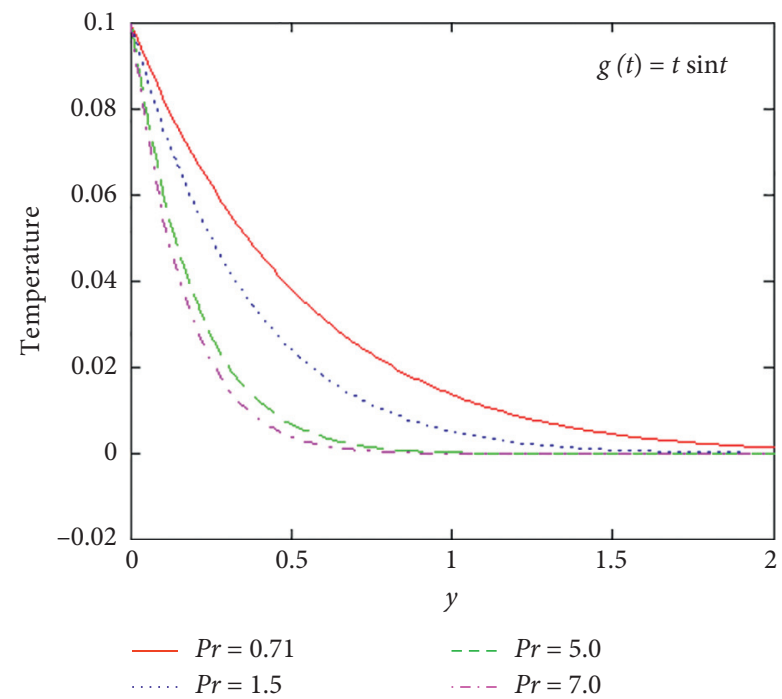

(f)

Figure 5: Profile of temperature for different values of $P_{r}$ and variation of time. 


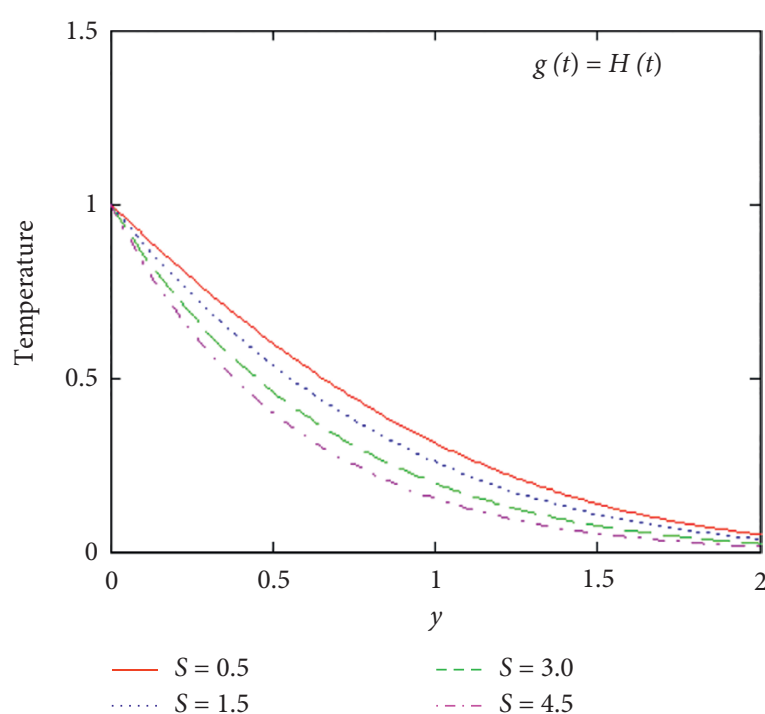

(a)

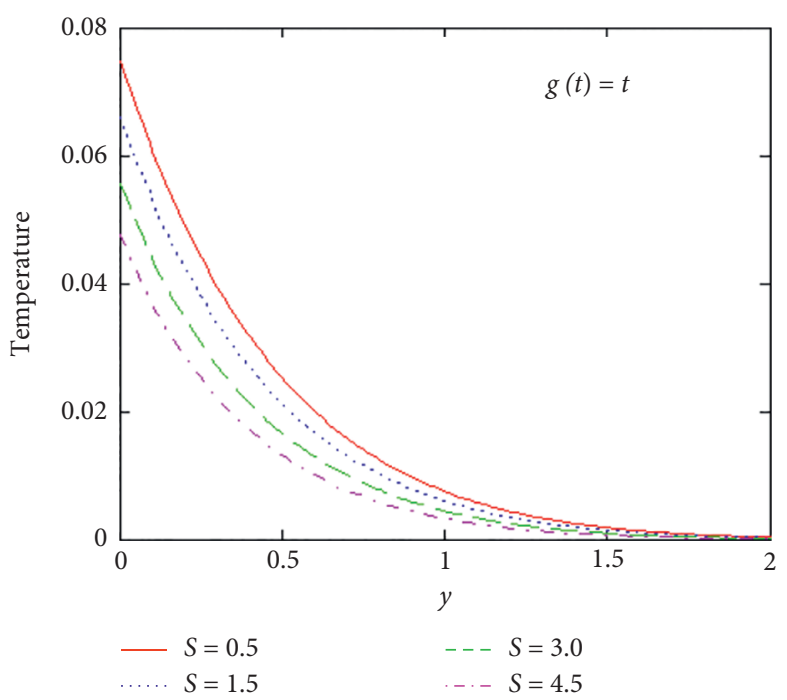

(c)

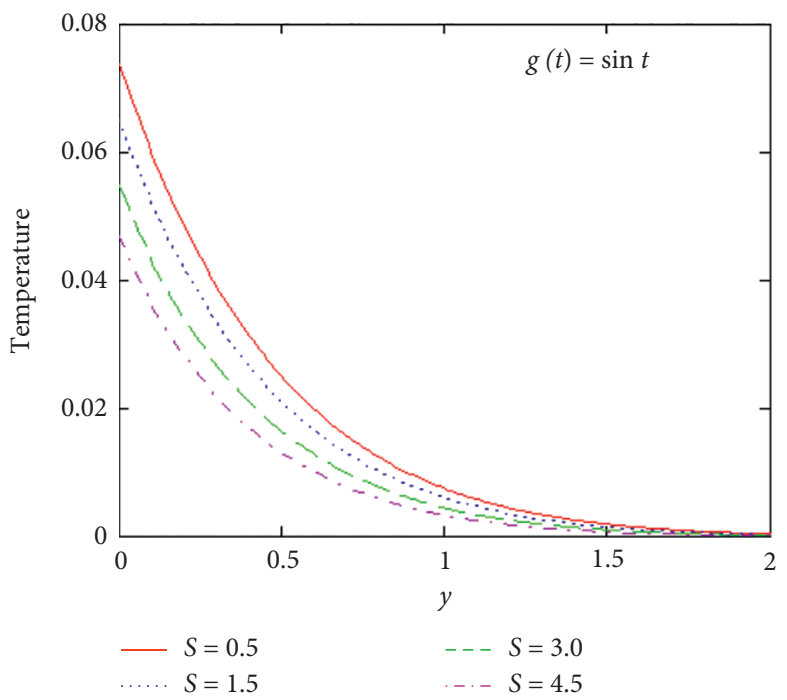

(e)

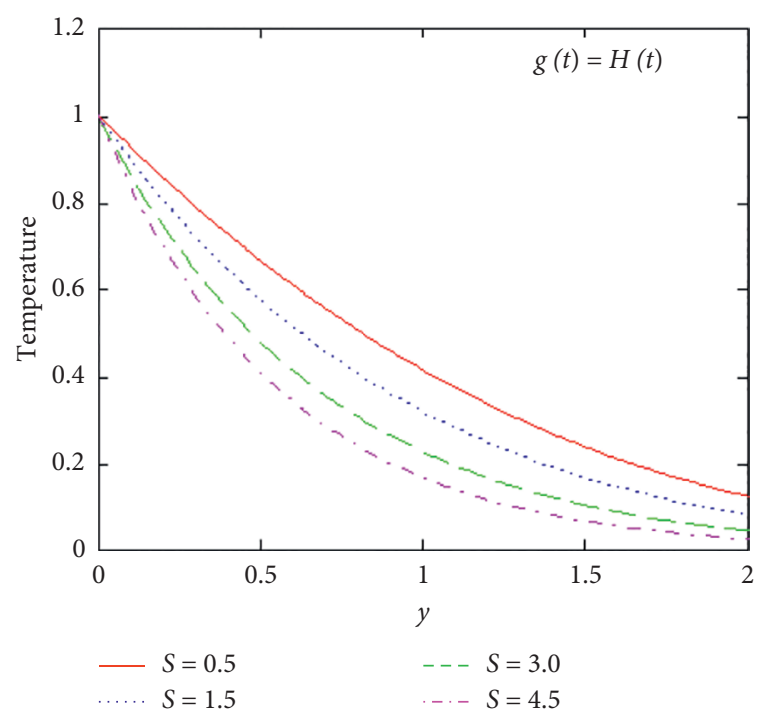

(b)

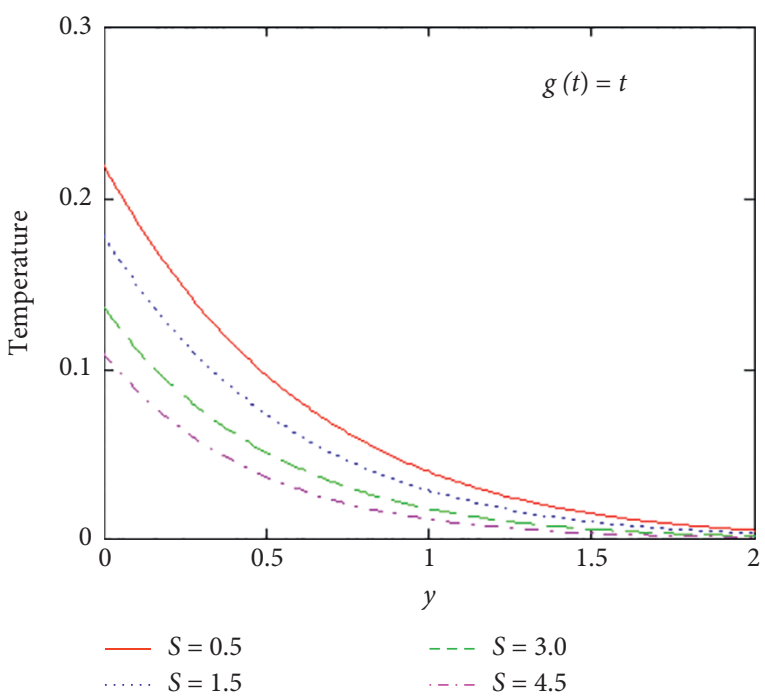

(d)

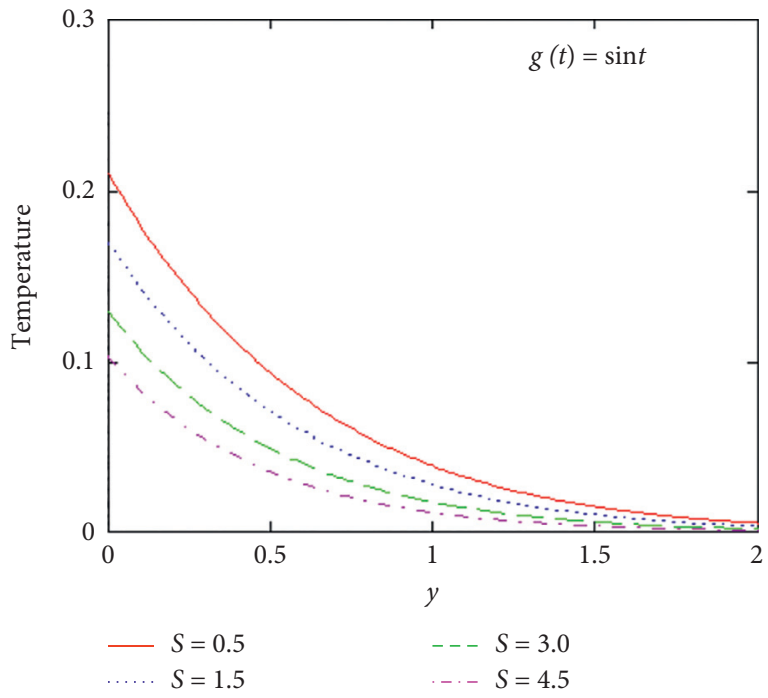

(f)

Figure 6: Continued. 


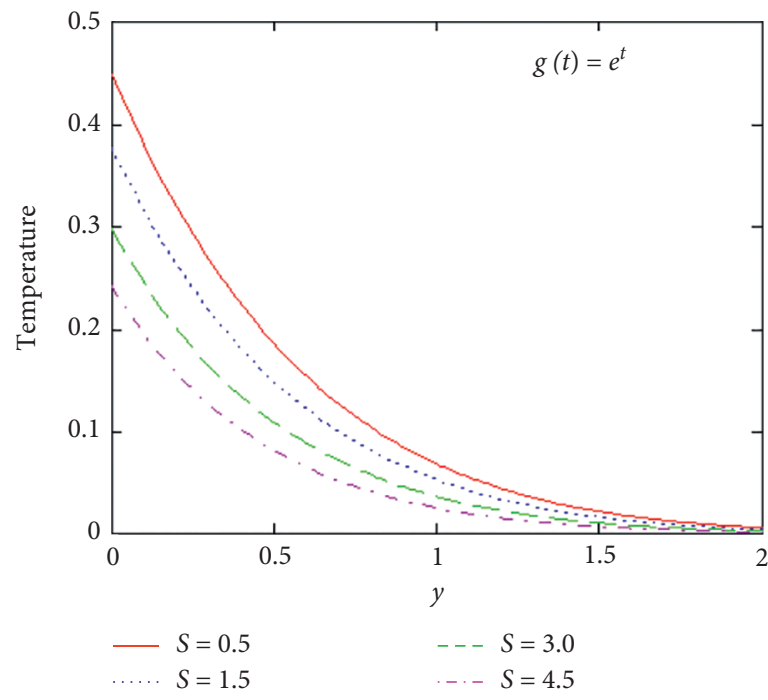

(g)

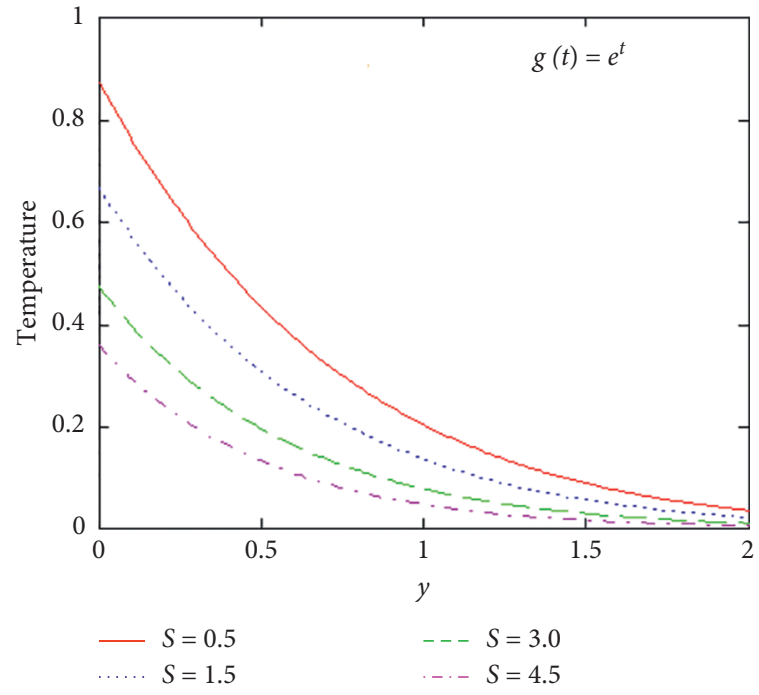

(h)

FIgURE 6: Profile of temperature for different values of $S$ and variation of time.

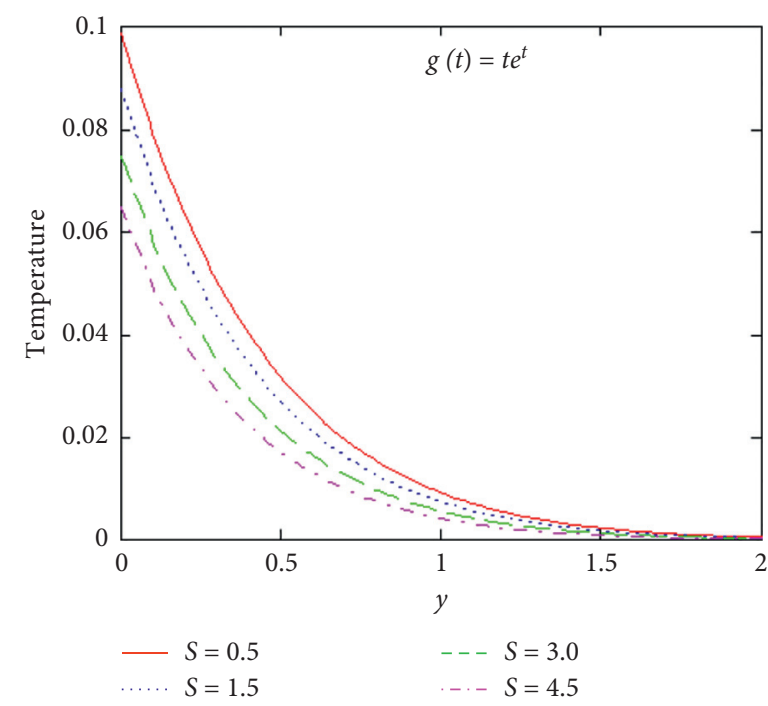

(a)

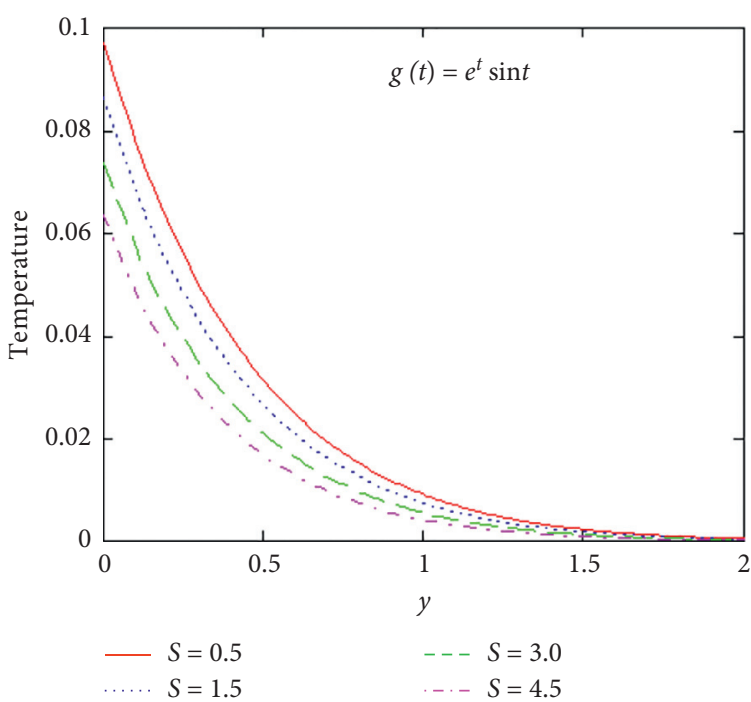

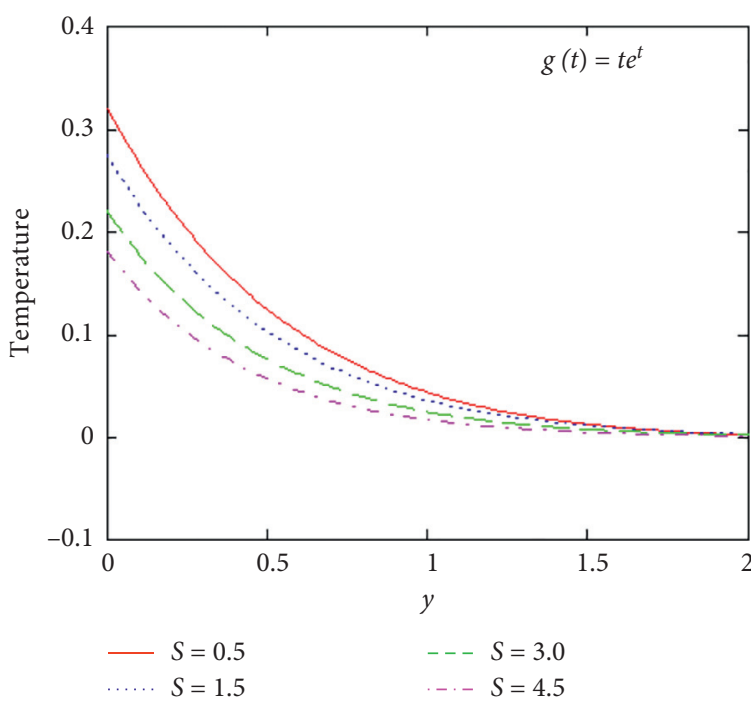

(b)

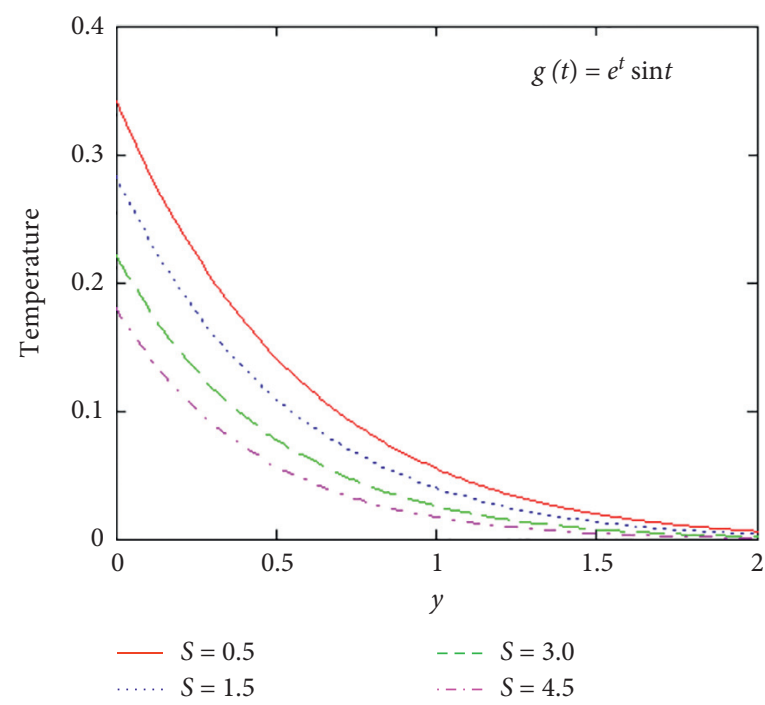

(d)

Figure 7: Continued. 


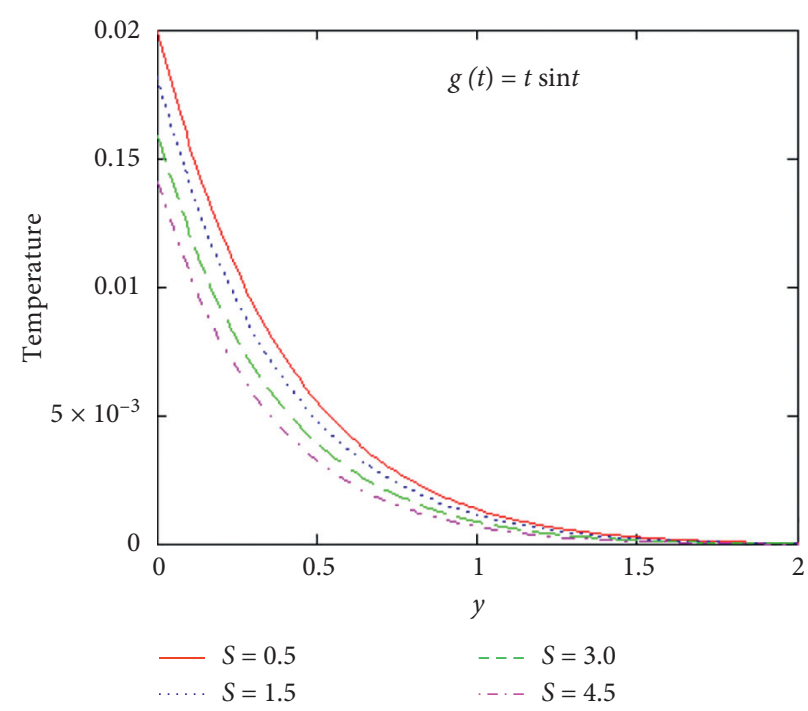

(e)

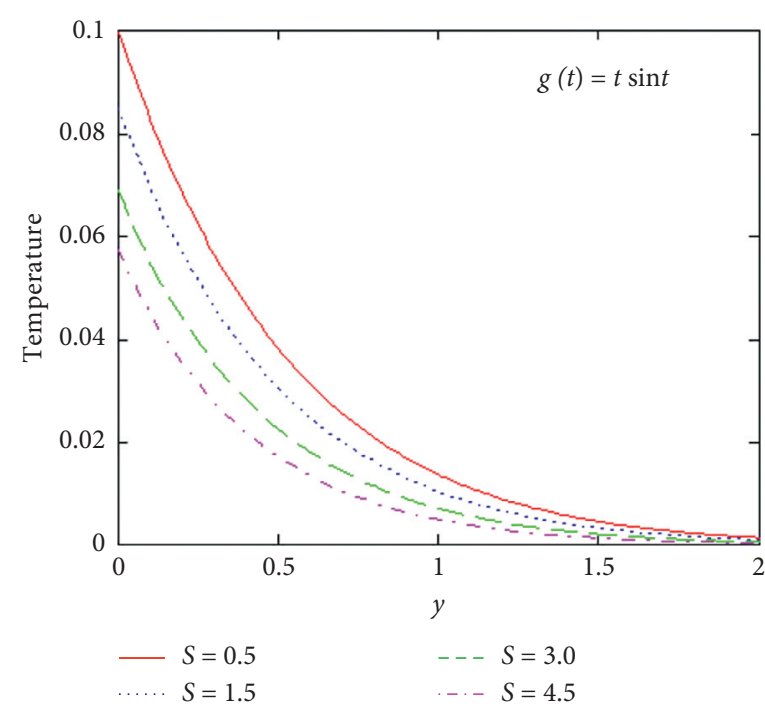

(f)

FIgURE 7: Profile of temperature for different values of $S$ and variation of time.
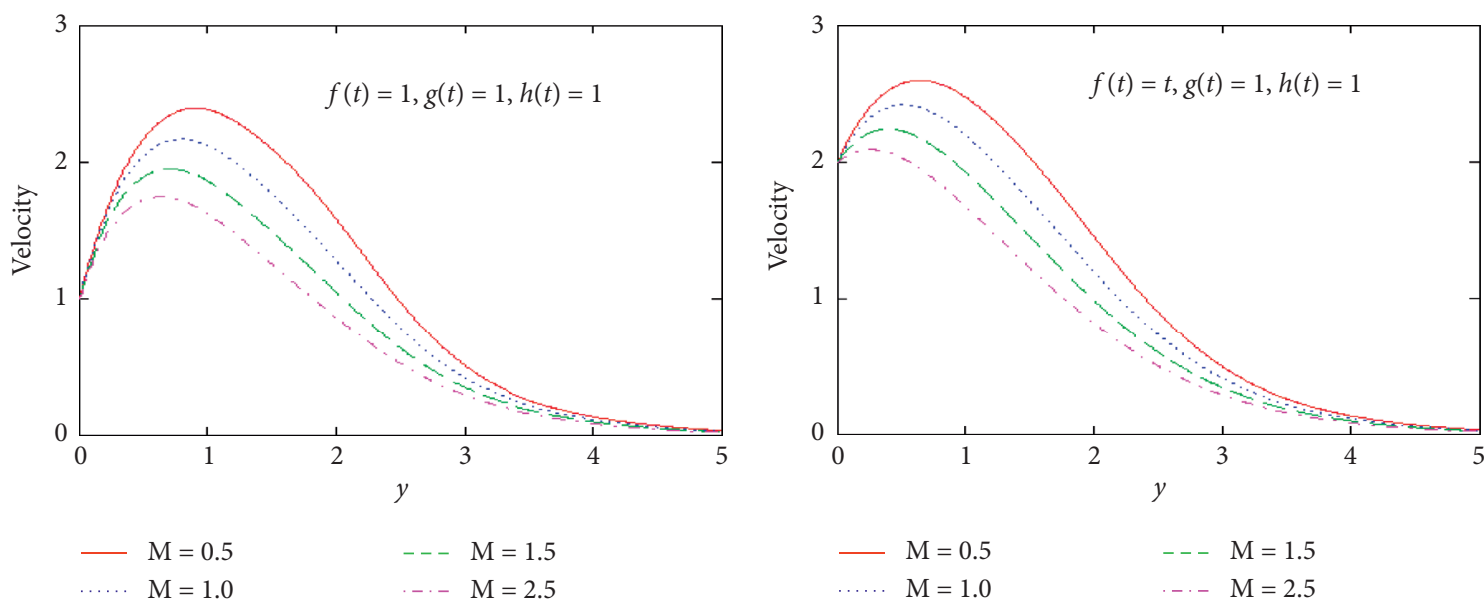

(a)

(b)
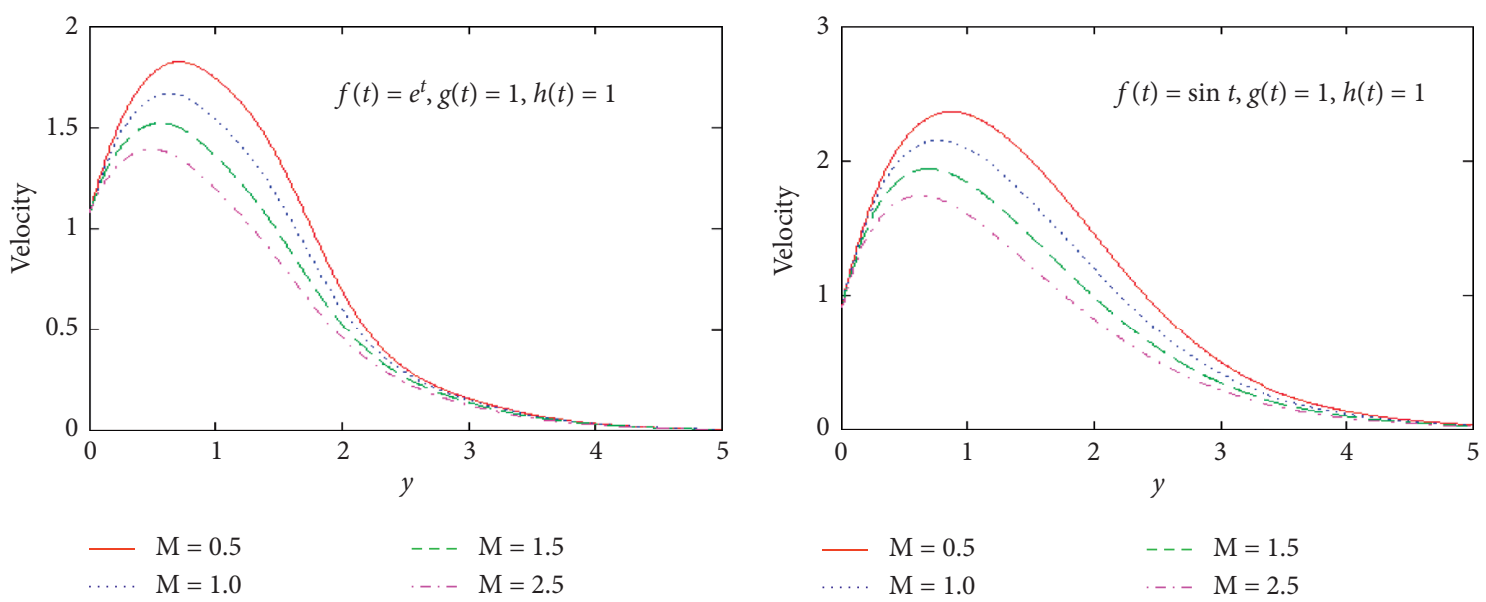

(c)

(d)

FIgURE 8: Continued. 


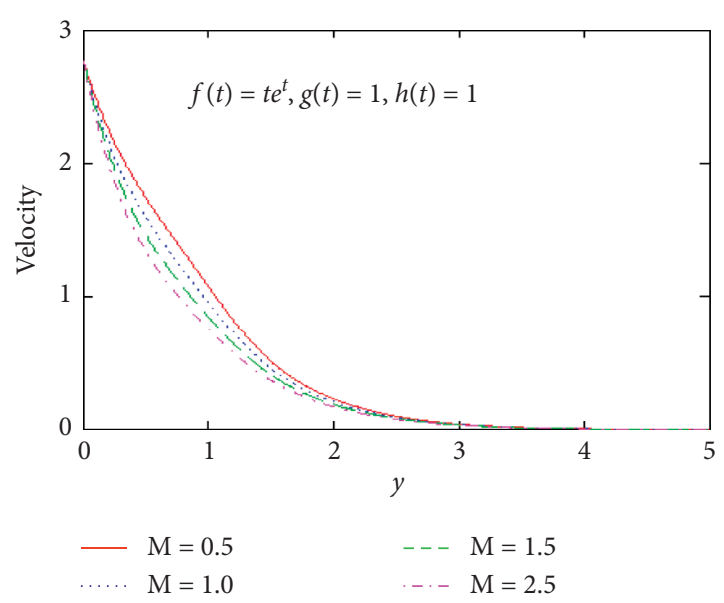

(e)

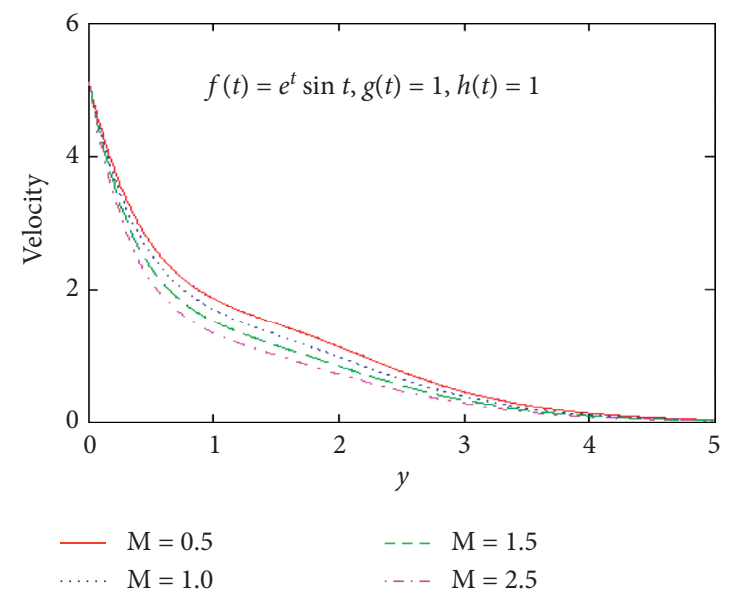

(f)

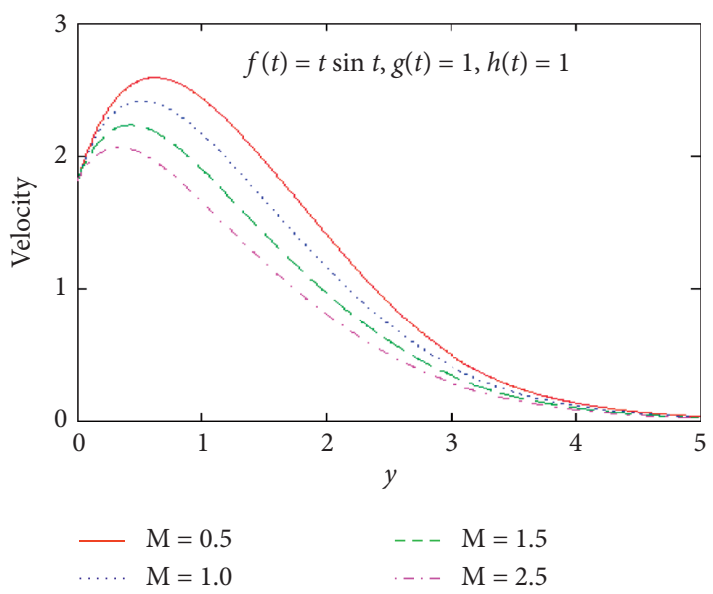

(g)

Figure 8: Profile of velocity for different values of $M$ and $P_{r}=0.71, \lambda=0.6, S=0.5, S_{c}=0.60, K_{c}=0.5, G_{T}=5.0, G_{C}=2.0$.

acceleration in fluid flow is due to the fact that buoyancy forces bcomes powerful than viscous forces.

Figures 12-14 show the chemical reaction $K_{c}$ parameter effects on velocity and concentration description. The increase in $K_{c}$ decreases the concentration and velocity profiles. Basically, chemical molecular diffusivity and species concentration drop with the higher values of $K_{c}$. The distribution in concentration falls at all the fluid flow field points with the rise in $K_{c}$. The curve for velocity for different values of the Maxwell fluid parameter $\lambda$ is plotted in Figure 15. It is observed that an increase in $\lambda$ produces a significant increase in the momentum boundary layer of the fluid which then increases the velocity. The rise in $\lambda$ will therefore correspond to a fall in fluid viscosity, resulting in it accelerating the flow and hence velocity rising. Further, an increase in $\lambda$ causes a rise in velocity near the plate surface. Although the trend is reversed away from the plate, the Newtonian fluid $(\lambda \longrightarrow 0)$ has a higher velocity.
In Figure 16, velocity profiles are plotted against the heat absorption parameter $S$ values. These curves show that the velocity is a decreasing function of parameter S. Furthermore, due to the absorption of heat, the fluid temperature diminishes and the thermal buoyancy force diminishes. These results have seen a fall in the velocity of a fluid with the increase in the values of $S$.

Figure 17 shows the behavior of velocity profile for different values of the parameters with a different choice of the function $f(t), g(t), h(t)$. For validation of our results, we consider some special cases of temperature profile already existing in literature and their graphical illustration is depicted in Figures 18-20. Figure 18 shows the temperature decrease with the increase in $P_{r}$ for the variation of time with $g(t)=1-e^{-t}$. The effects of the heat absorption parameter can be observed in Figure (19) which depicts the decline in temperature. The impacts of $g(t)=1-a e^{-b t}$ for different choices of $a$ and $b$ are explained in Figure 20. We see the decline in temperature with the increasing values of $a$ and $b$. 


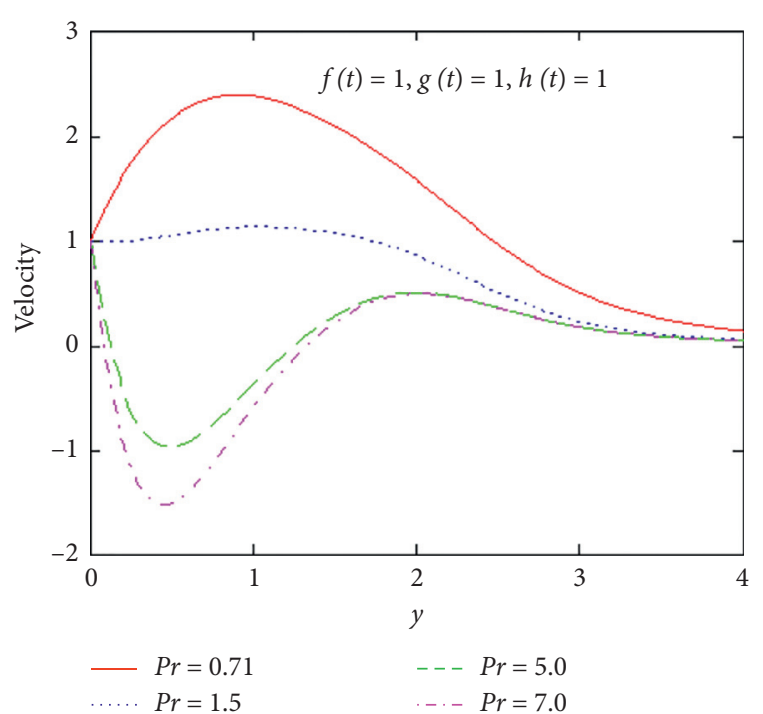

(a)

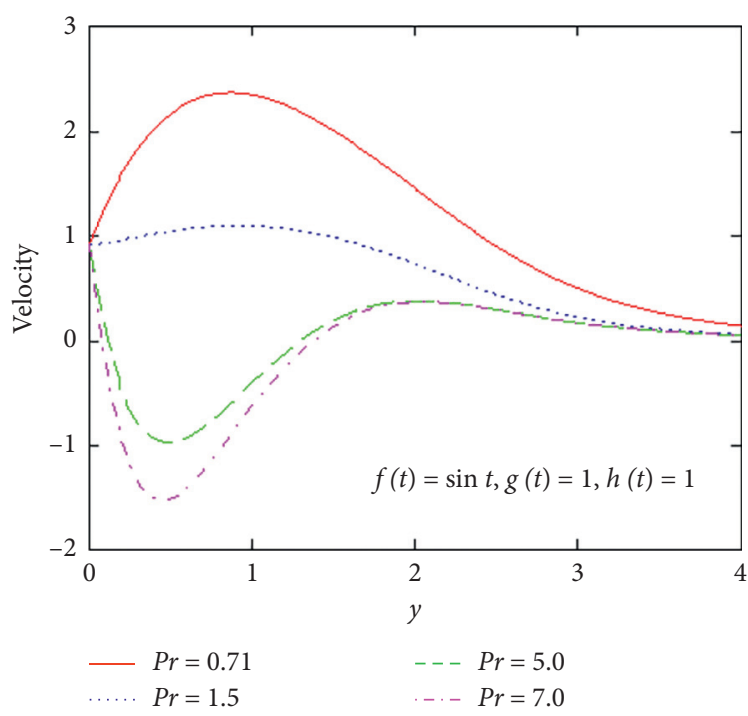

(c)

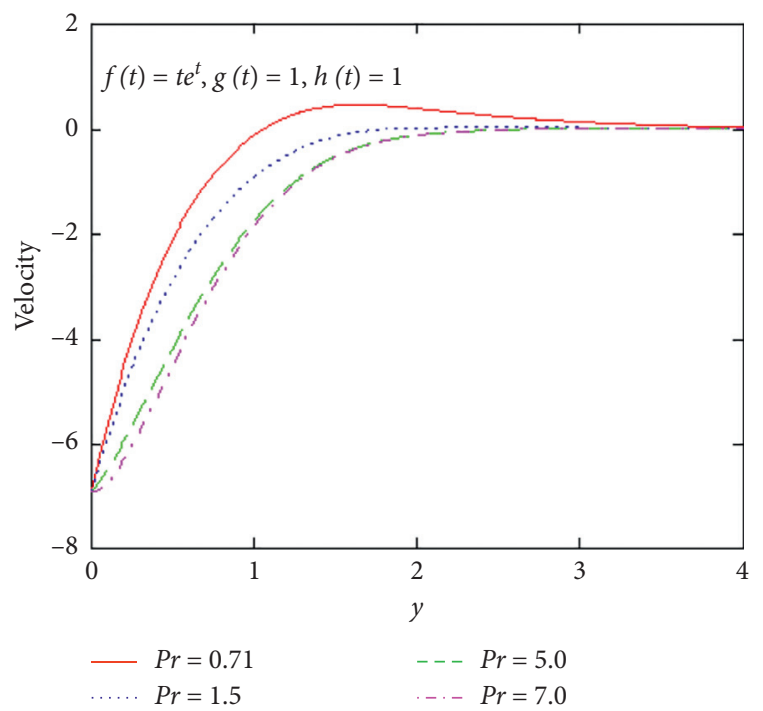

(e)

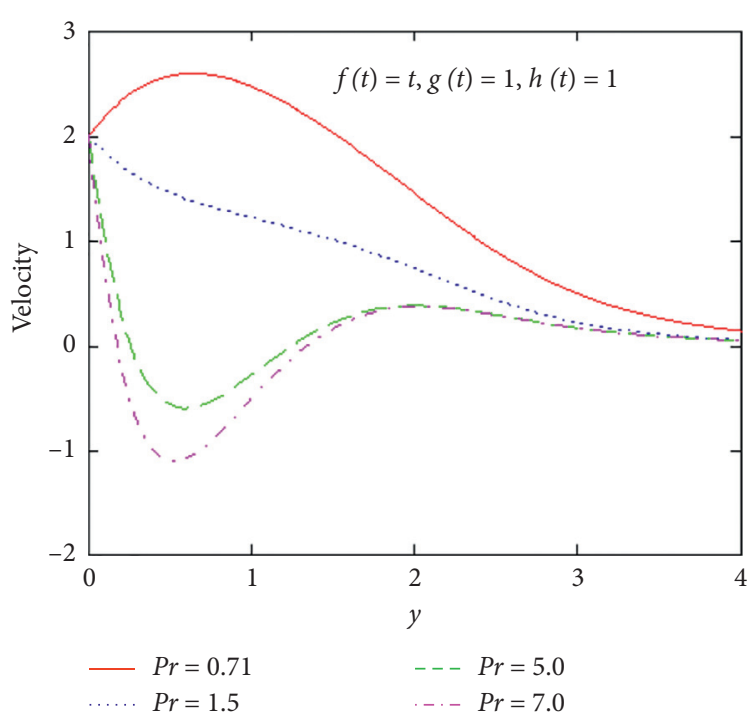

(b)

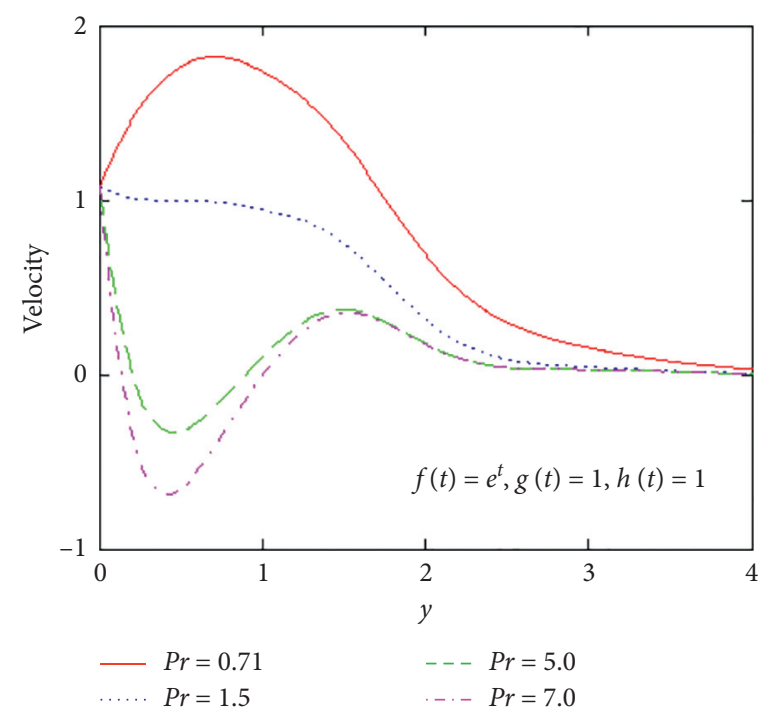

(d)

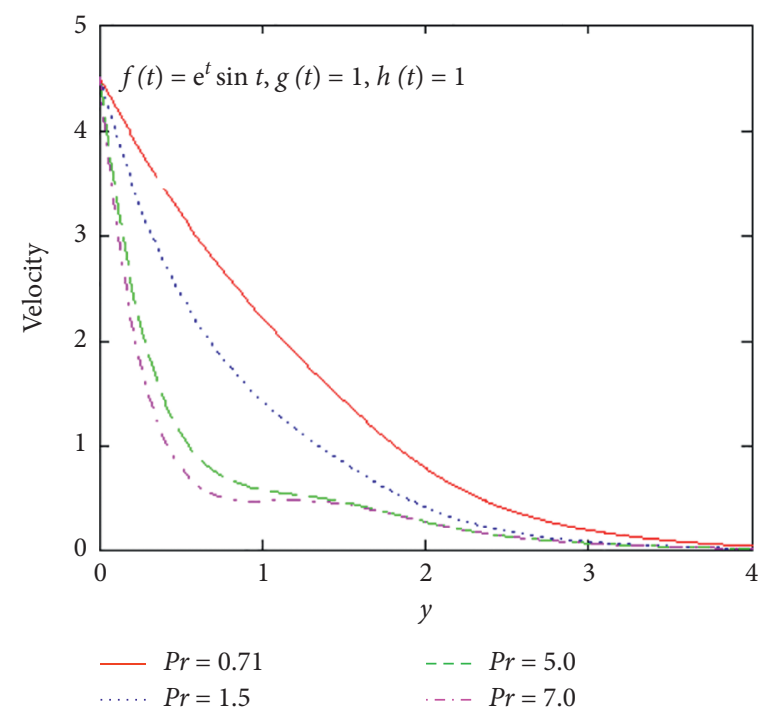

(f)

Figure 9: Continued. 


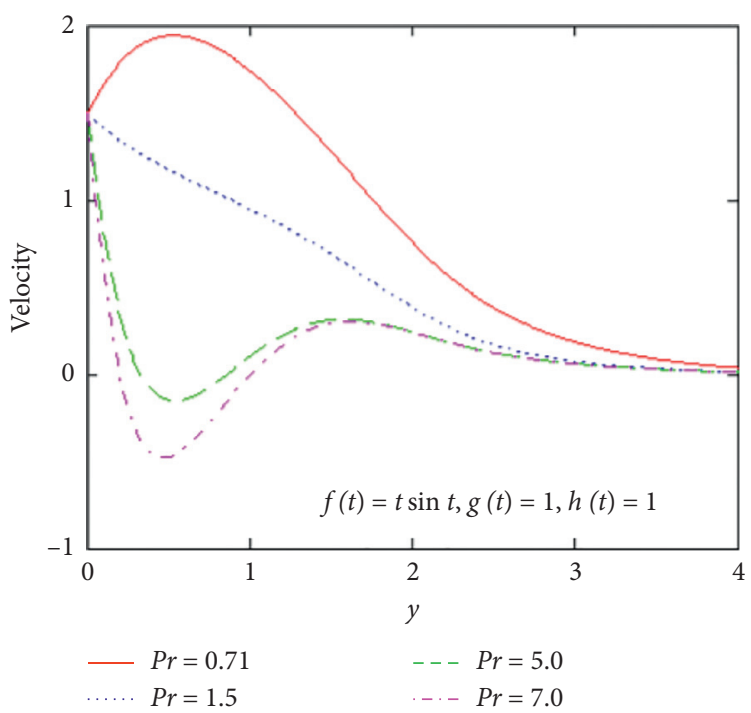

(g)

Figure 9: Profile of velocity for different values of $P_{r}$ and $M=0.5, \lambda=0.6, S=0.5, S_{c}=0.60, K_{c}=0.5, G_{T}=5.0, G_{C}=2.0$.

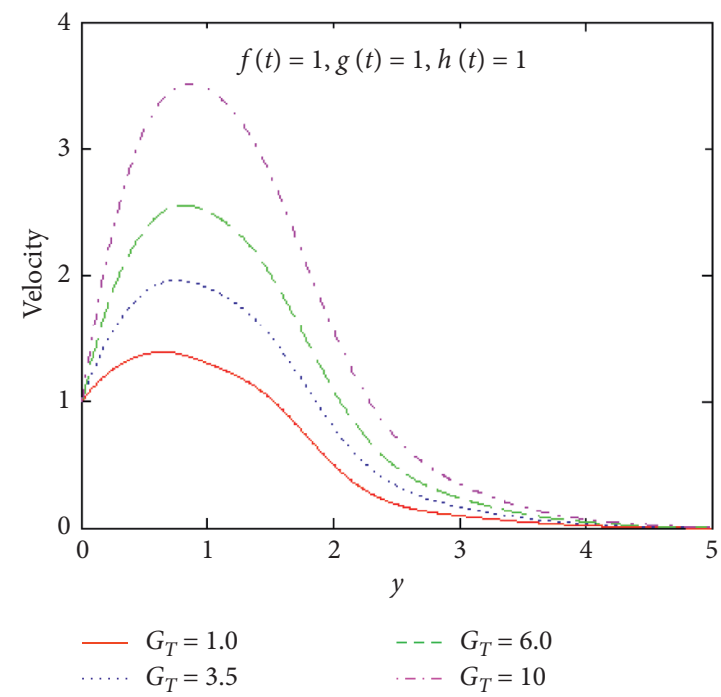

(a)

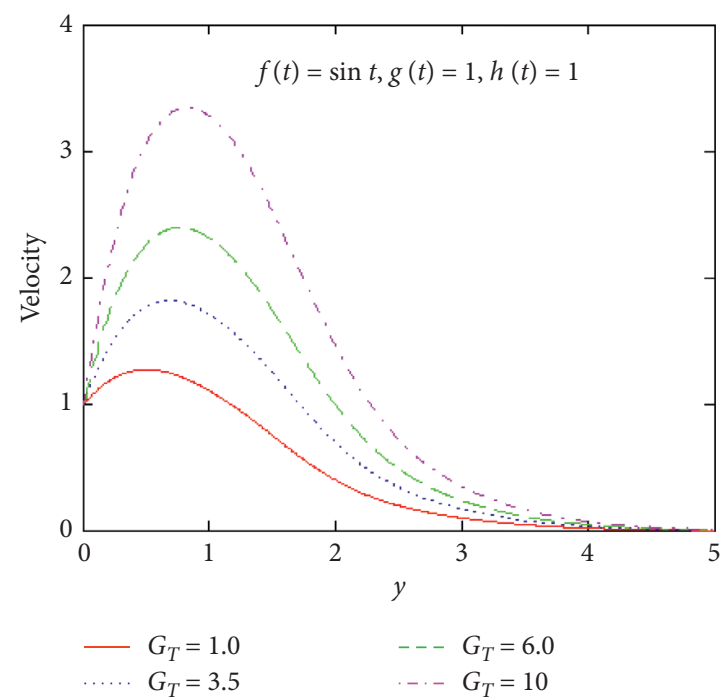

(c)

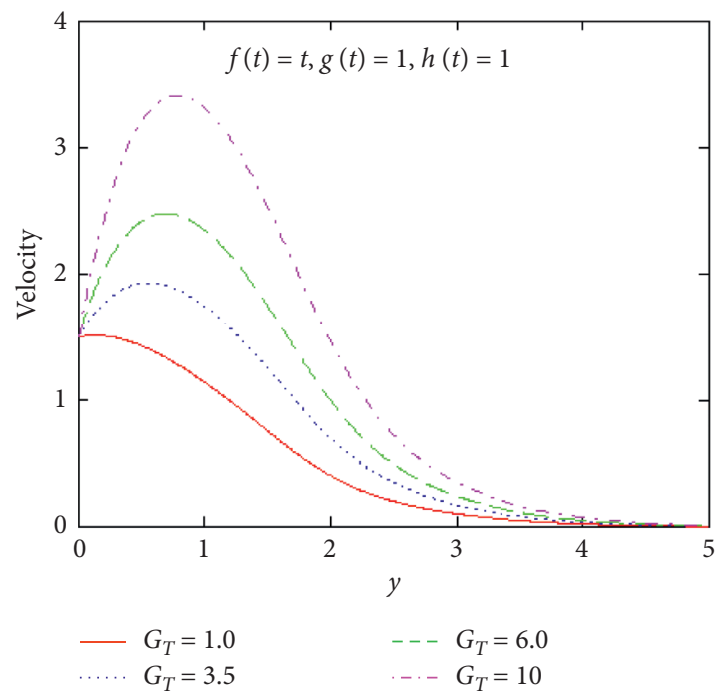

(b)

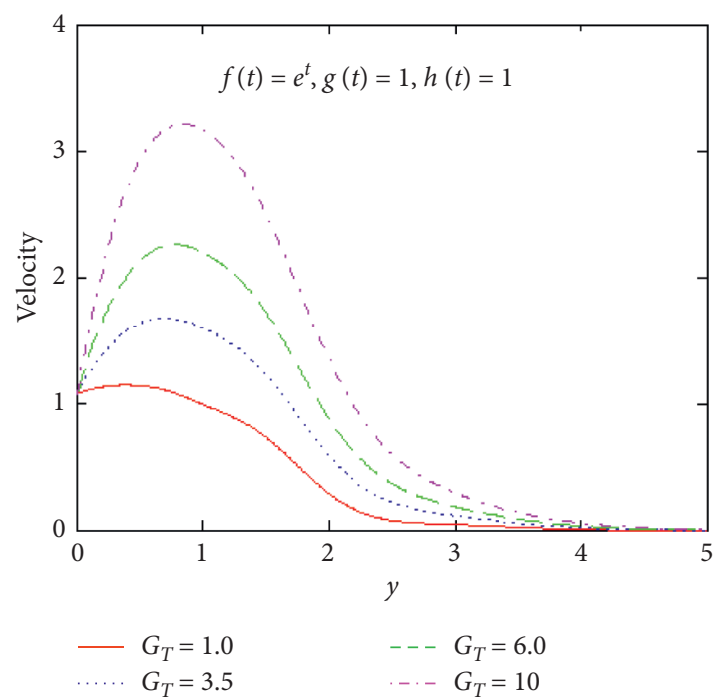

(d)

Figure 10: Continued. 


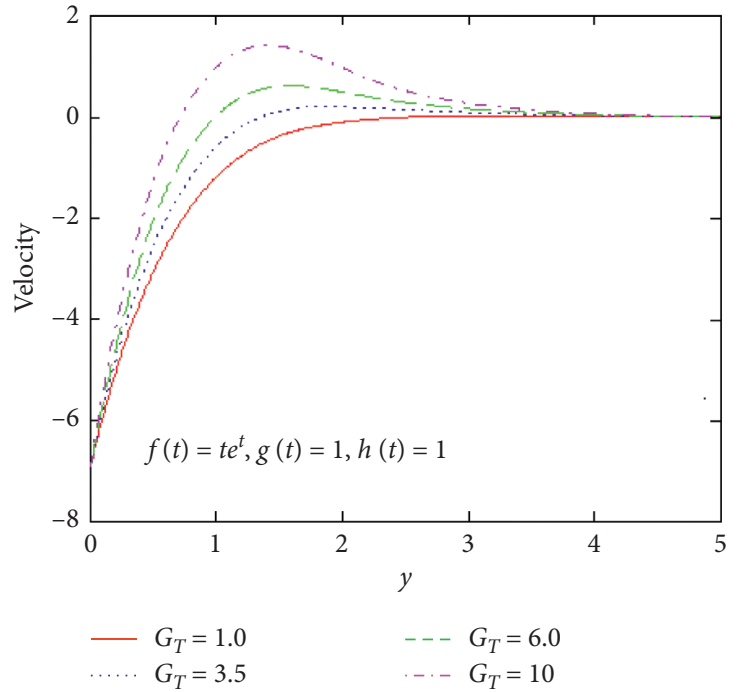

(e)

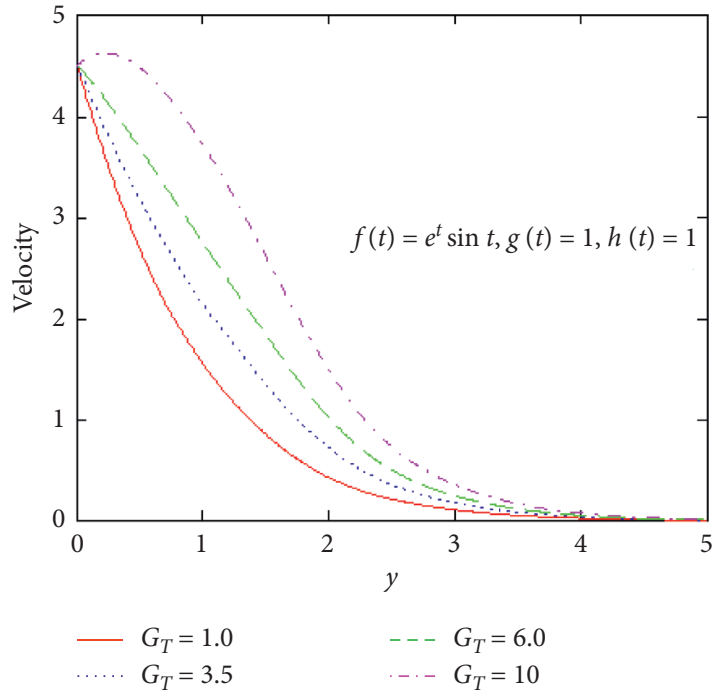

(f)

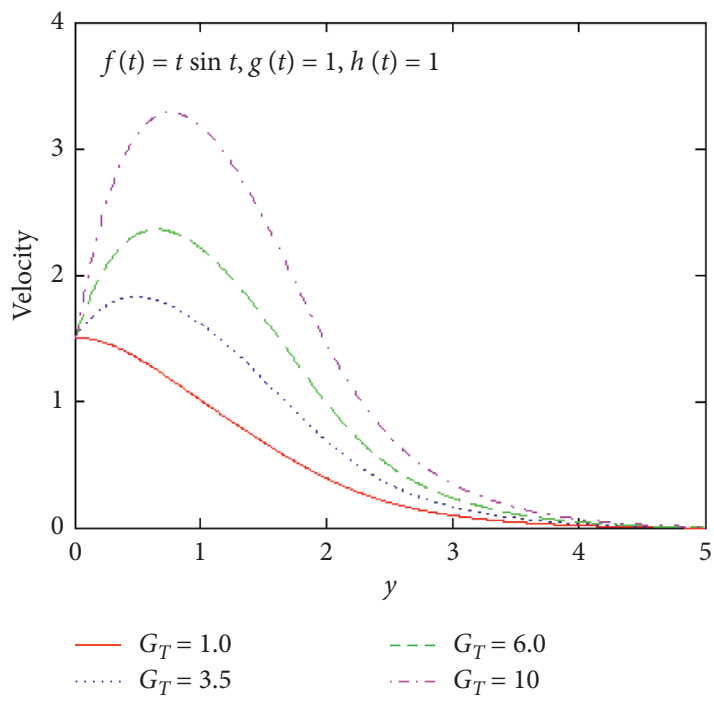

(g)

Figure 10: Profile of velocity for different values of $G_{T}$ and $M=0.2, \lambda=0.6, S=1.0 .5, K_{c}=0.5, S_{c}=0.60, G_{C}=2.0, P_{r}=0.71$.
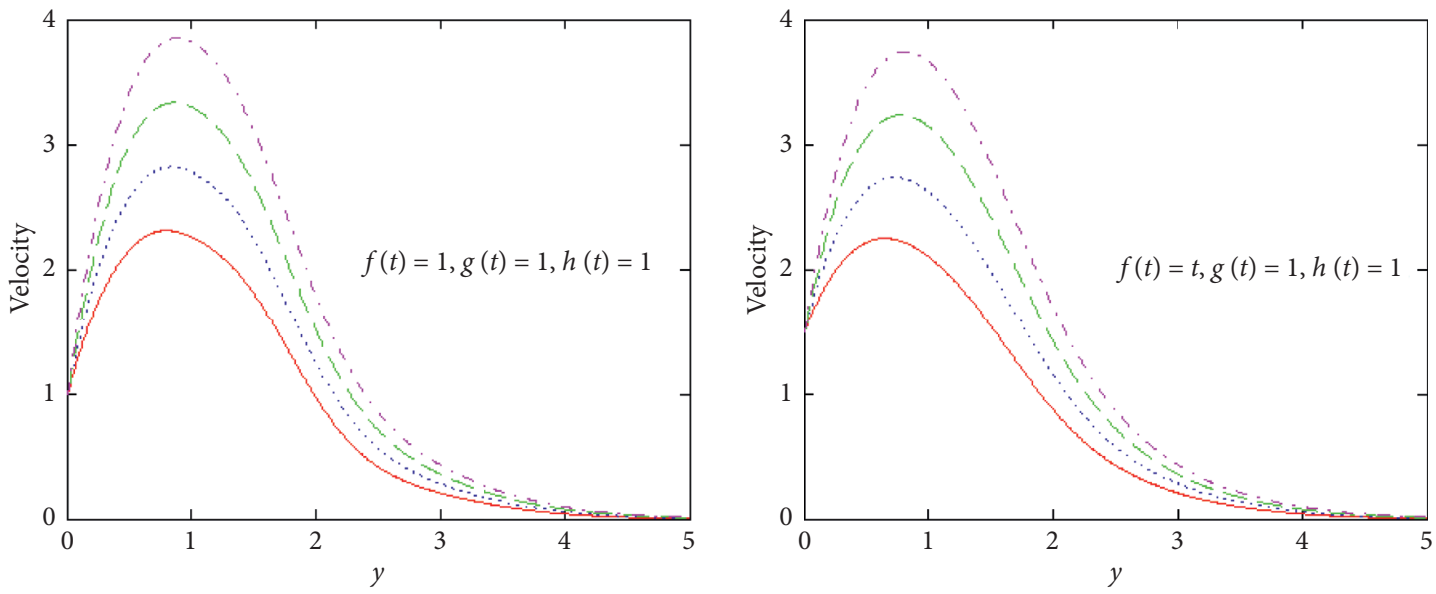

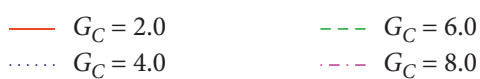

(a)

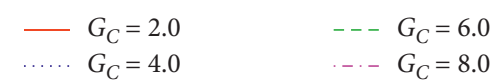

(b)

Figure 11: Continued. 

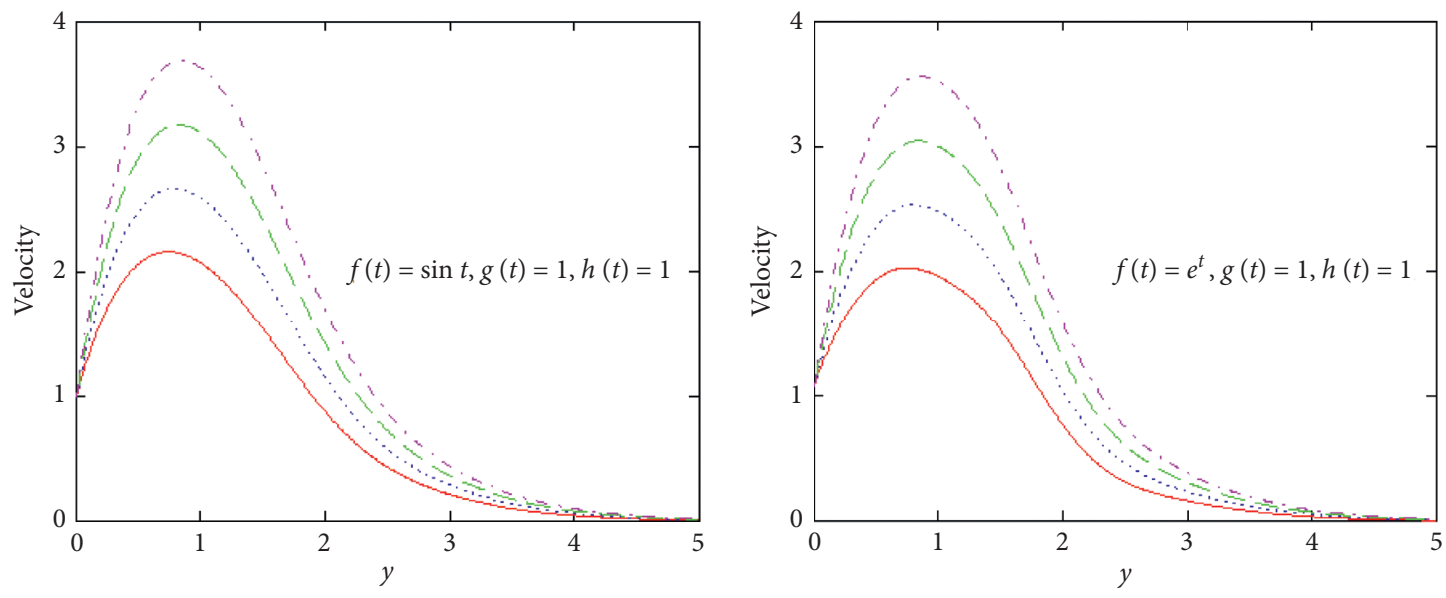

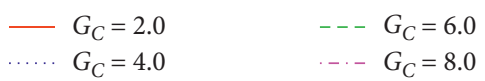

(c)

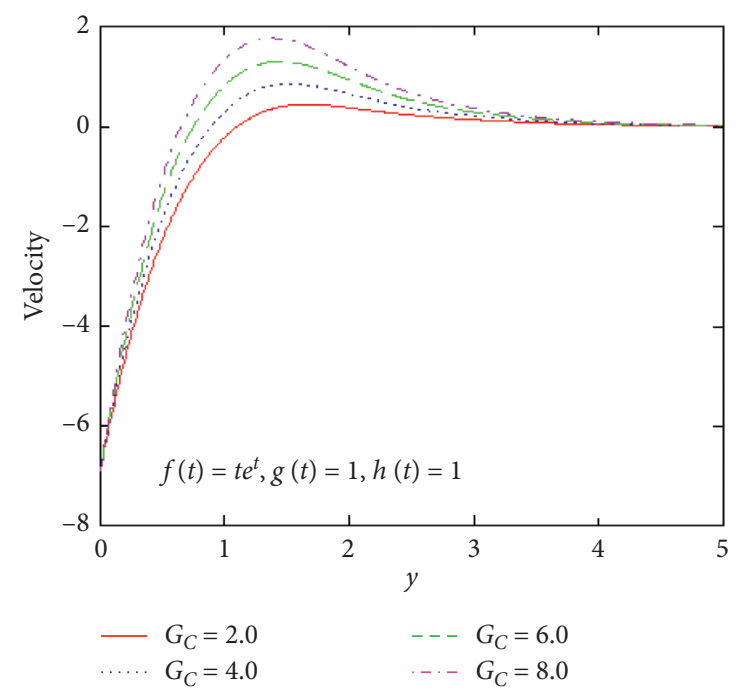

(e)

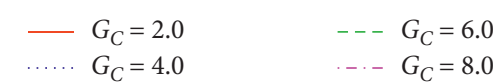

(d)

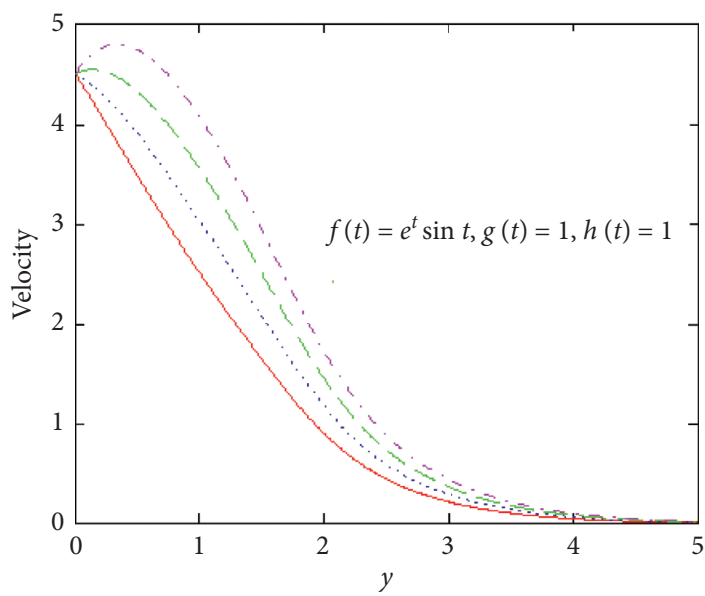

$-G_{C}=2.0$

$G_{C}=4.0$

- - $G_{C}=6.0$

-. $G_{C}=8.0$

(f)

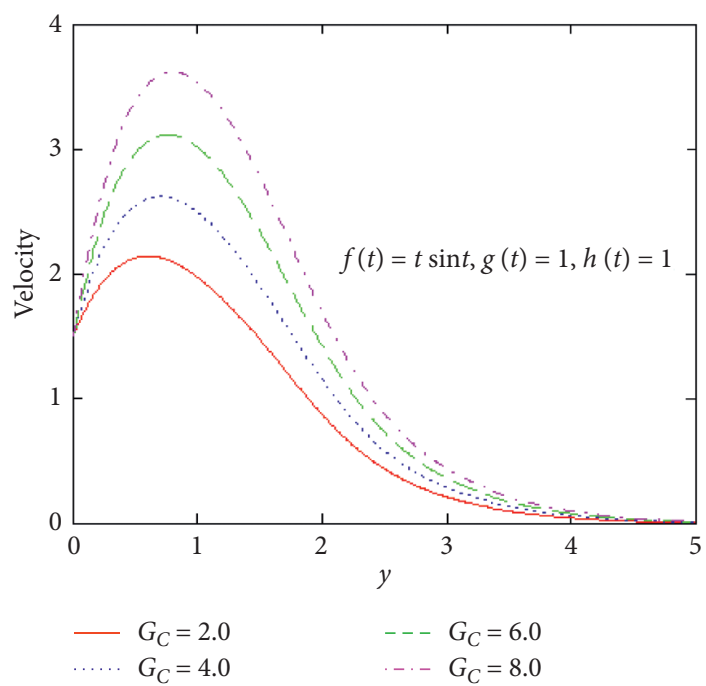

(g)

Figure 11: Profile of velocity for different values of $G_{C}$ and $M=0.2, \lambda=0.6, S=0.5, K_{c}=0.5, S_{c}=0.60, G_{T}=5.0, P_{r}=0.71$. 


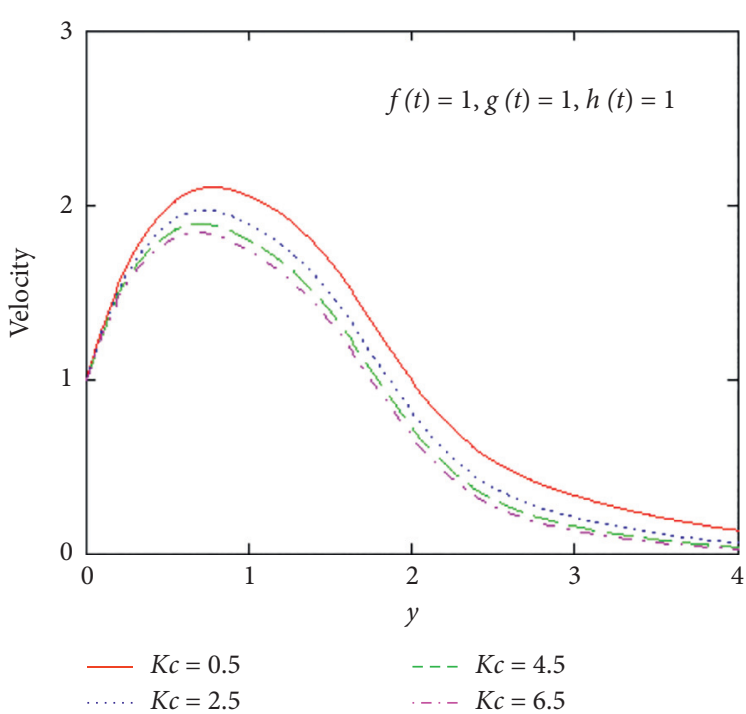

(a)

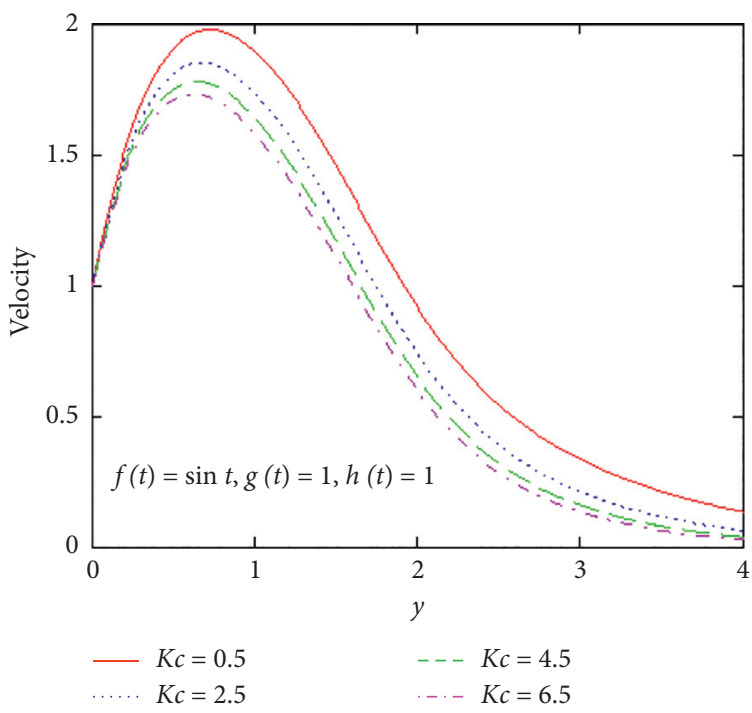

(c)

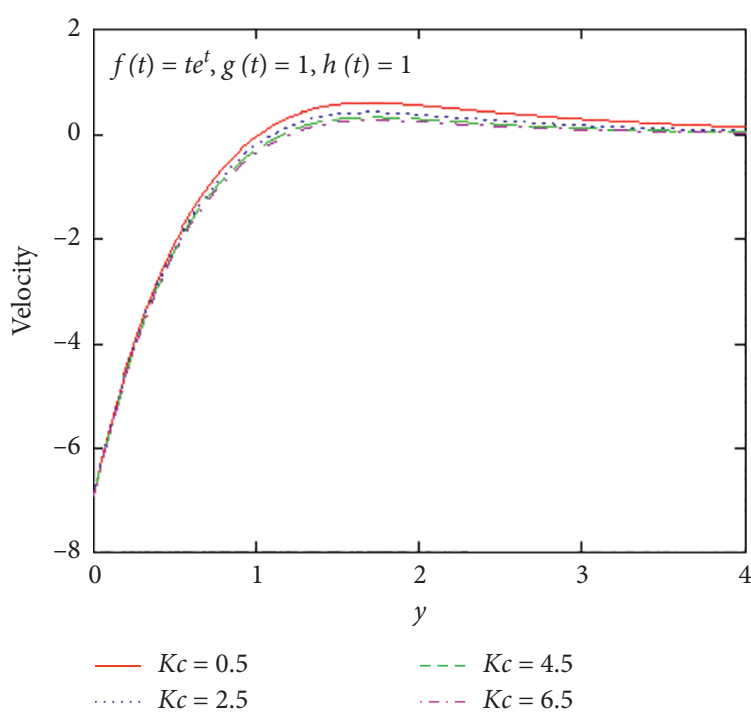

(e)

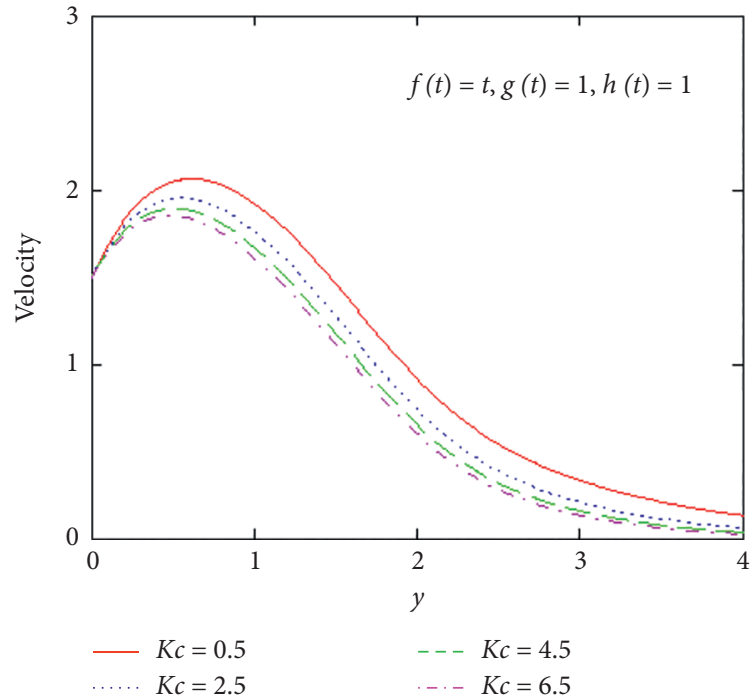

(b)

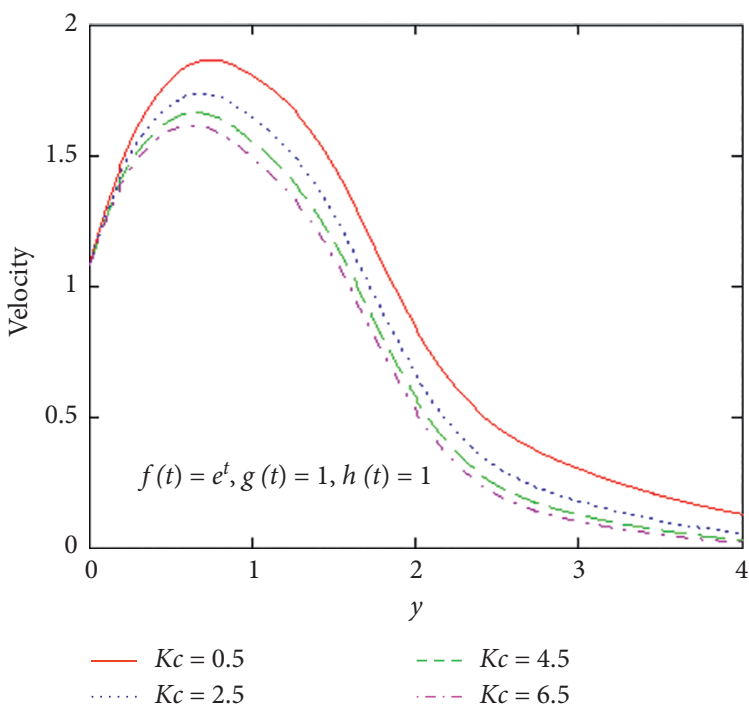

(d)

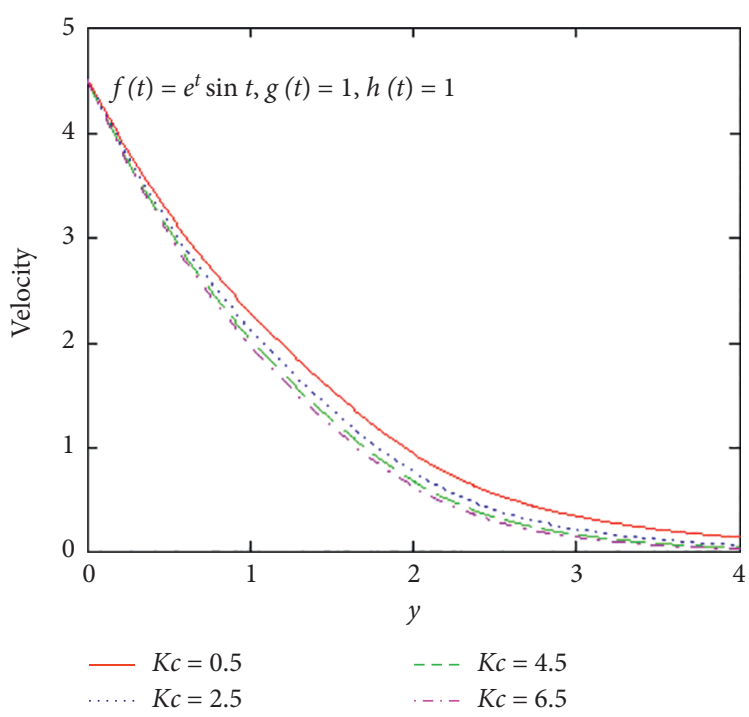

(f)

FIgURE 12: Continued. 


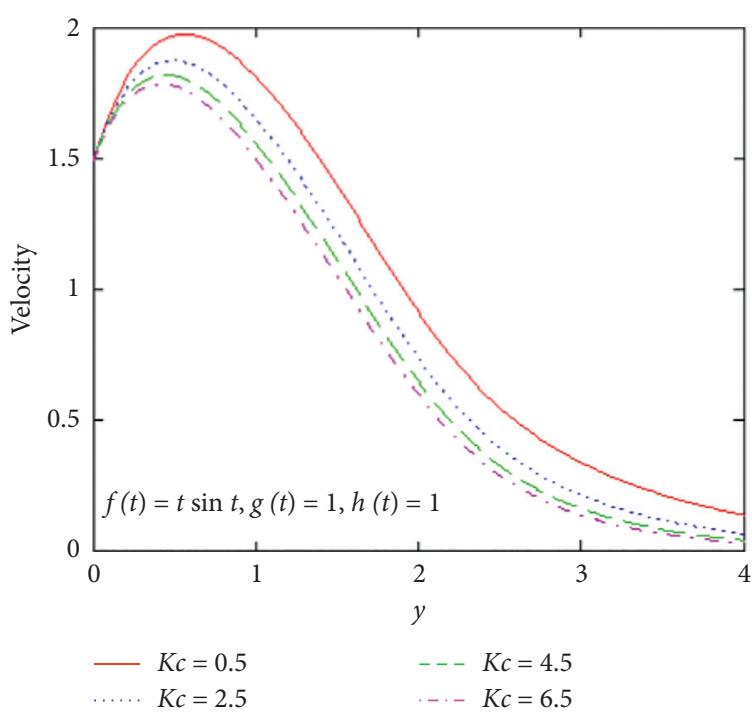

(g)

Figure 12: Profile of velocity for different values of $K_{c}$ and $M=0.5, \lambda=0.6, S=0.5, S_{c}=0.60, G_{T}=5.0, G_{C}=2.0, P_{r}=0.71$.

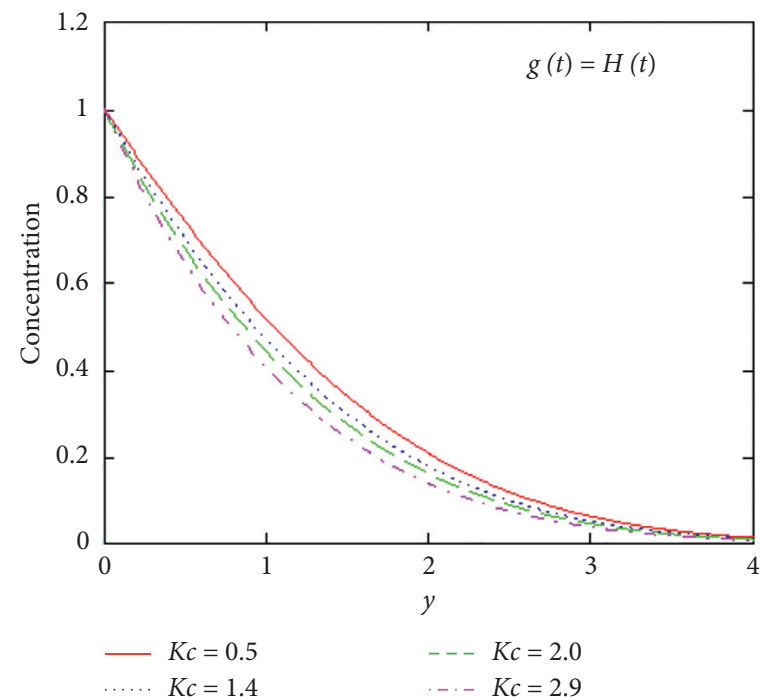

(a)

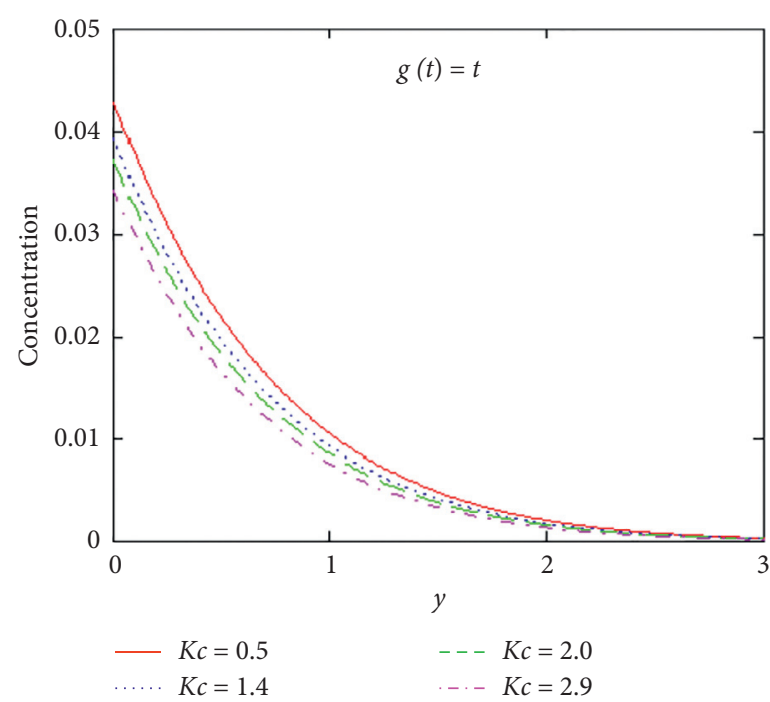

(c)

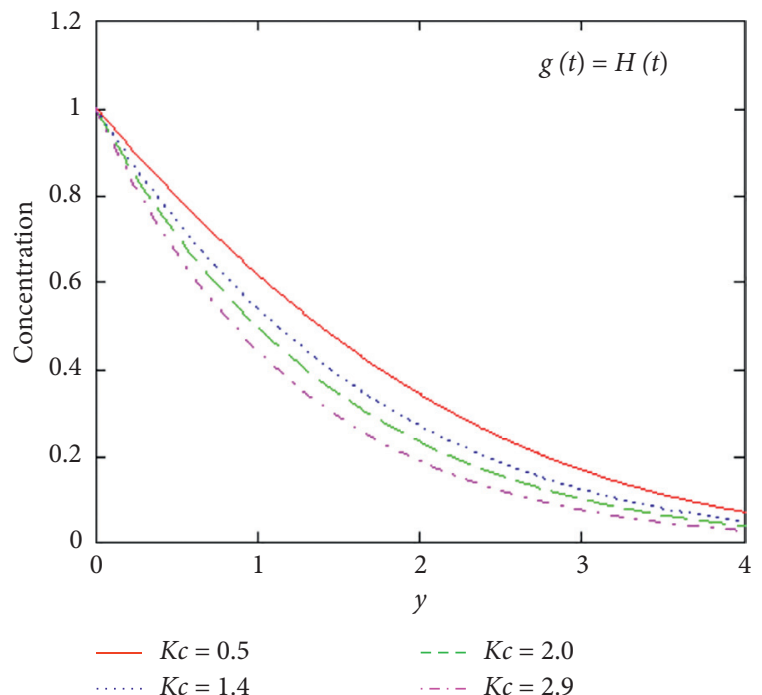

(b)

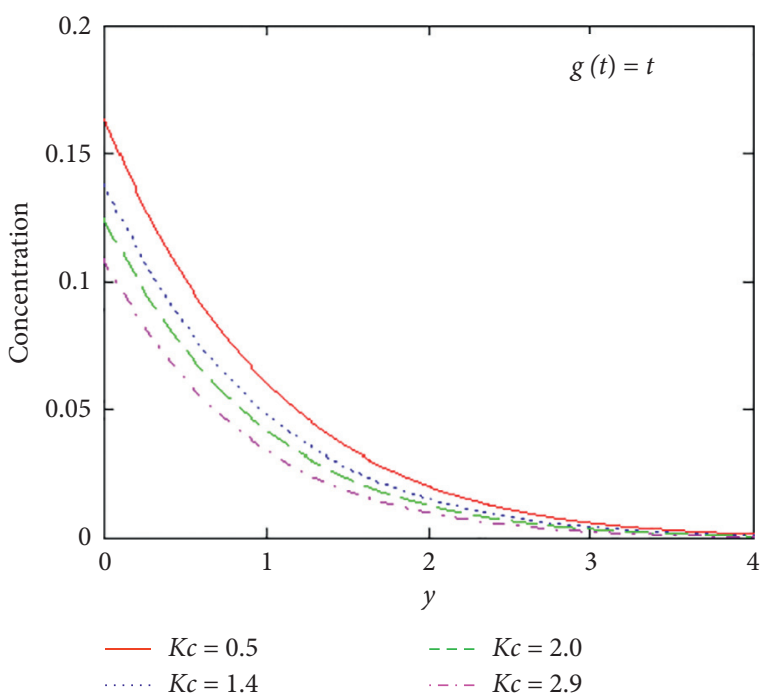

(d)

FIgURE 13: Continued. 


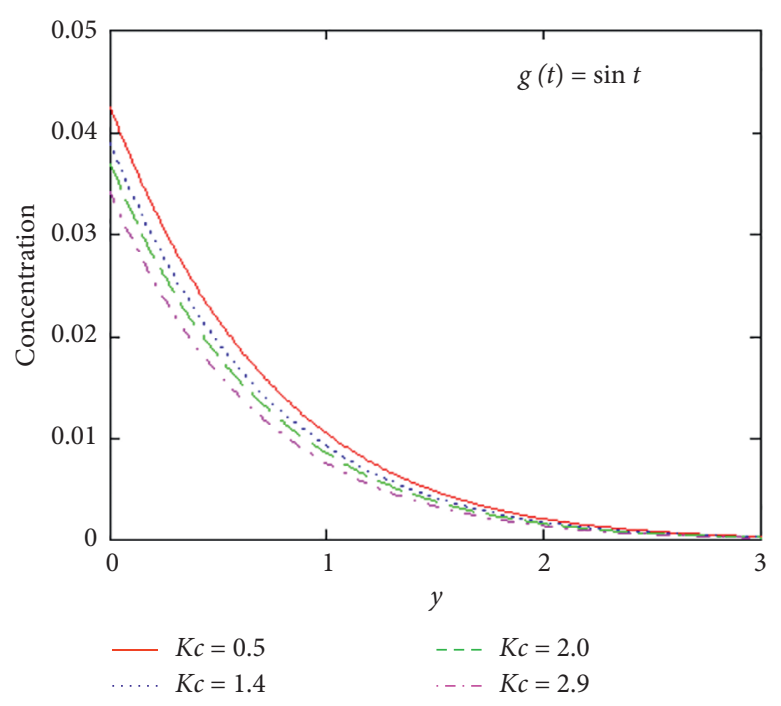

(e)

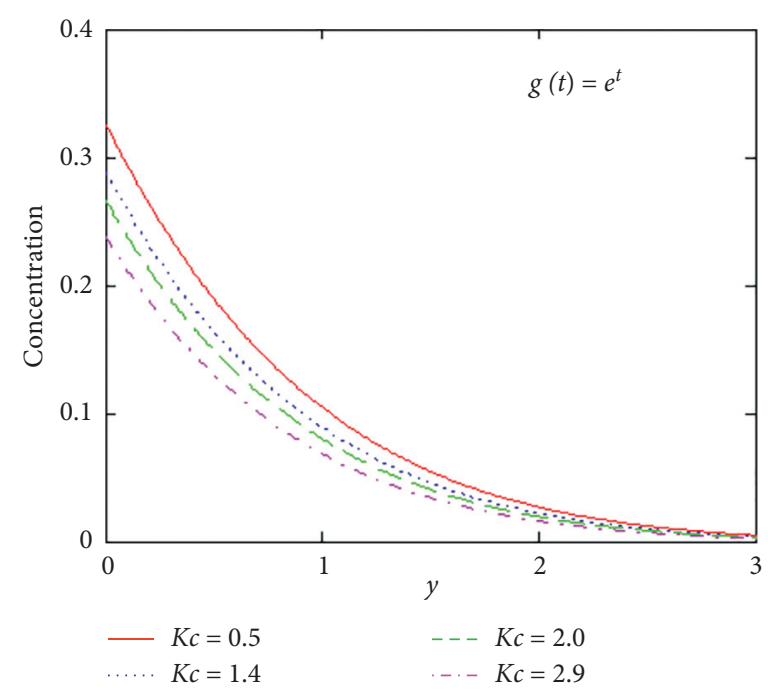

(g)

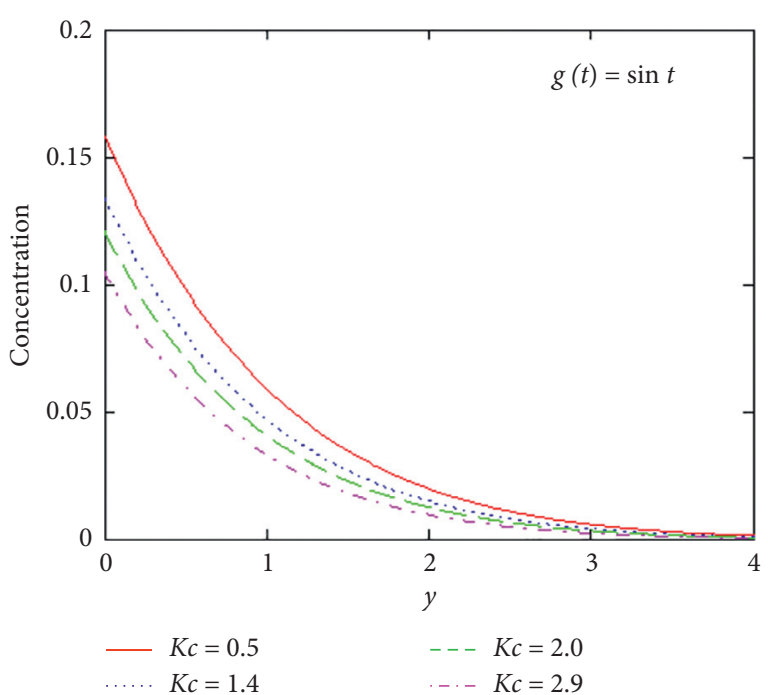

(f)

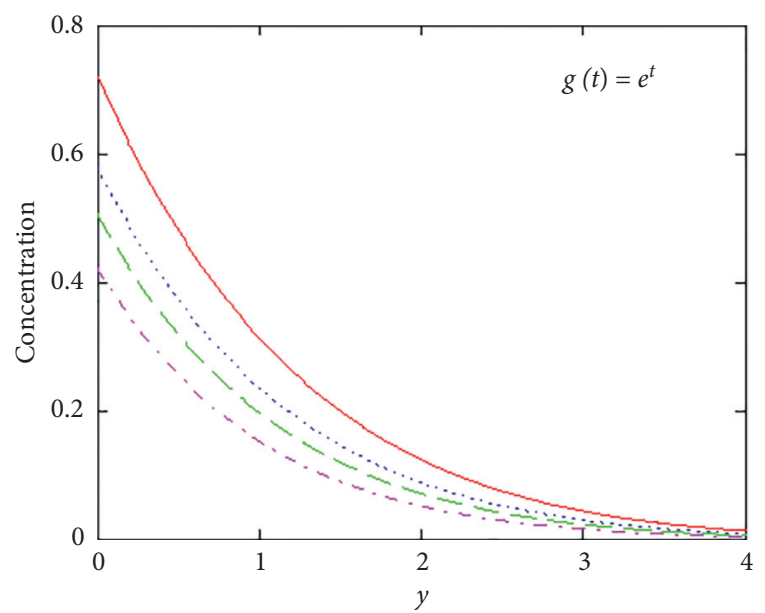

(h)

Figure 13: Profile of concentration for different values of $K_{c}$ and variation of time.

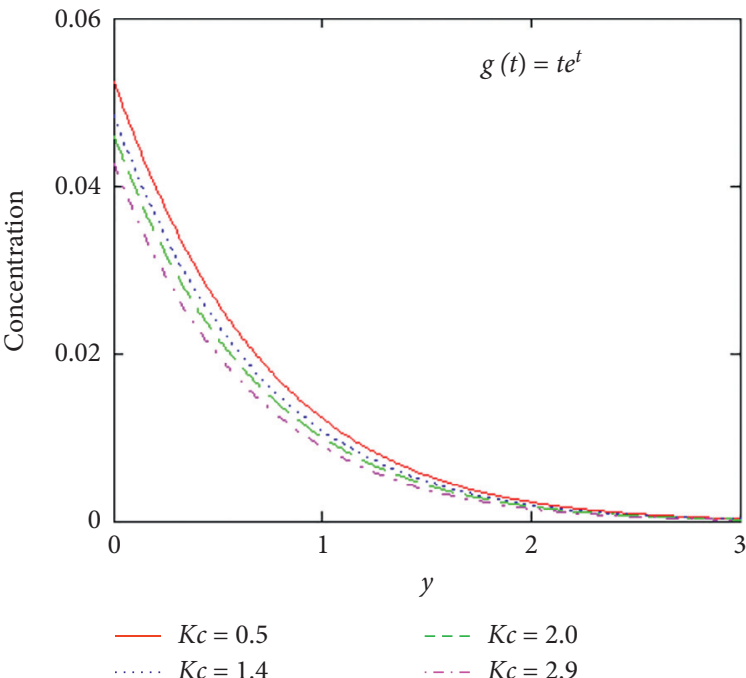

(a)

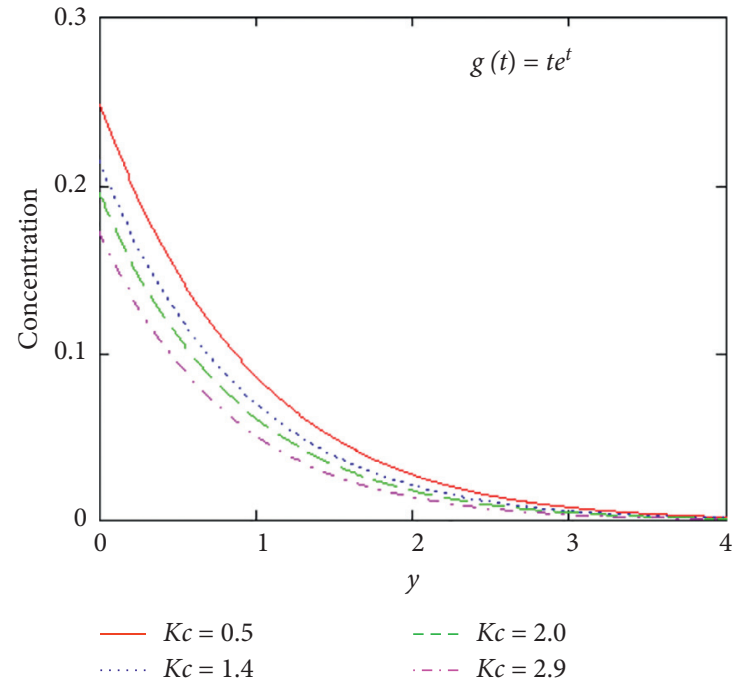

(b)

FIgURE 14: Continued. 


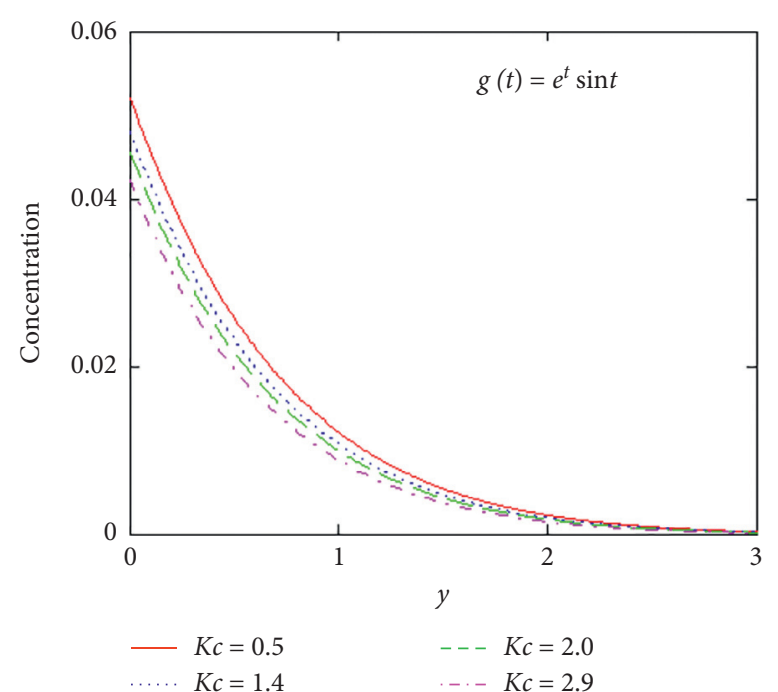

(c)

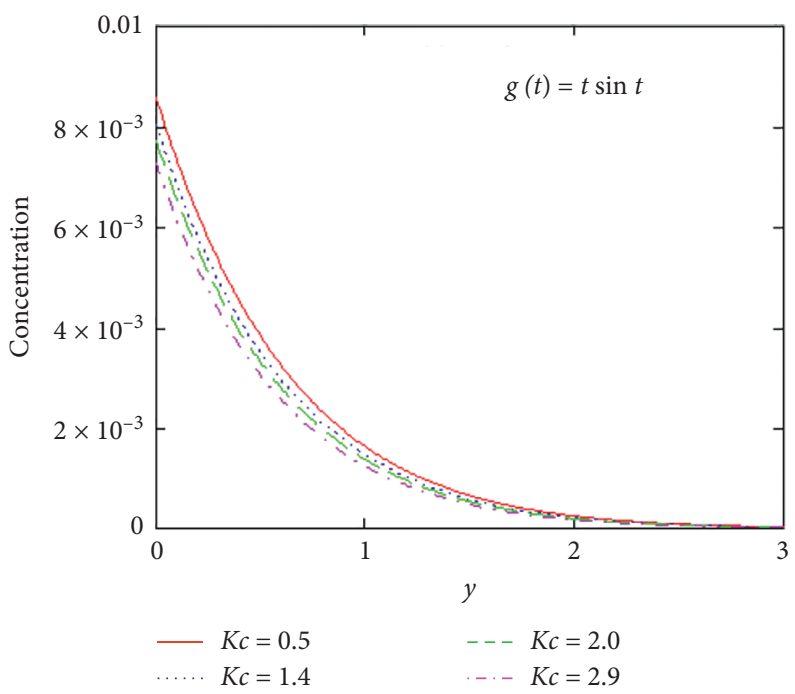

(e)

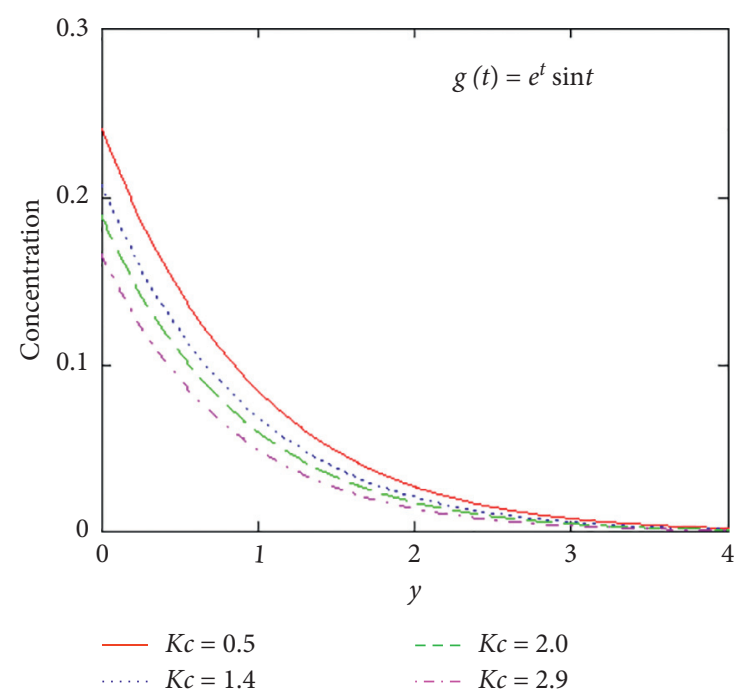

(d)

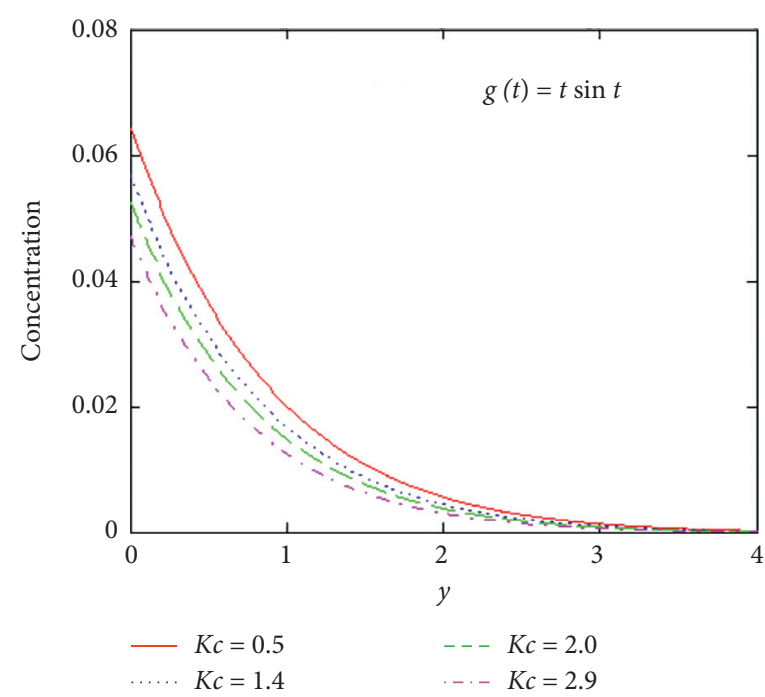

(f)

Figure 14: Profile of concentration for different values of $K_{c}$ and variation of time.

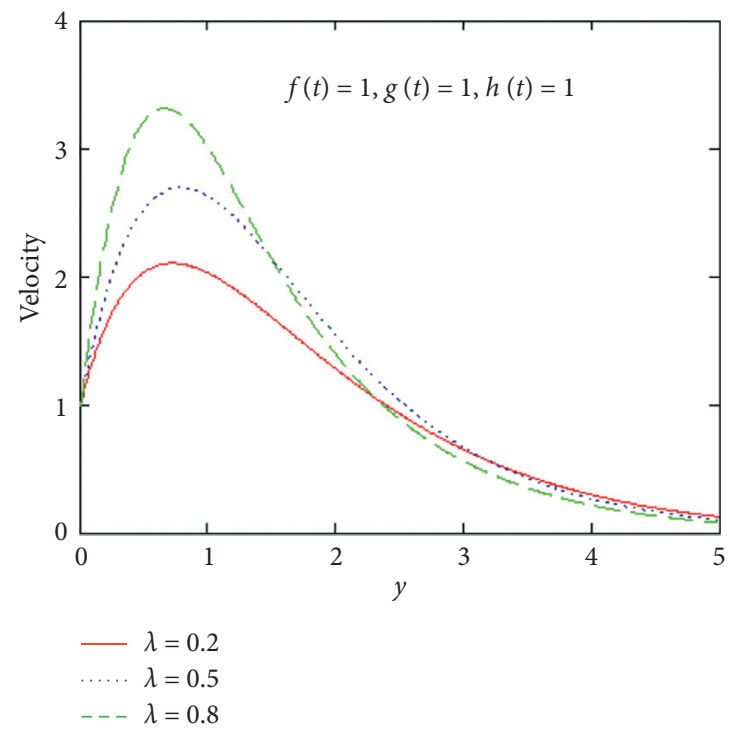

(a)

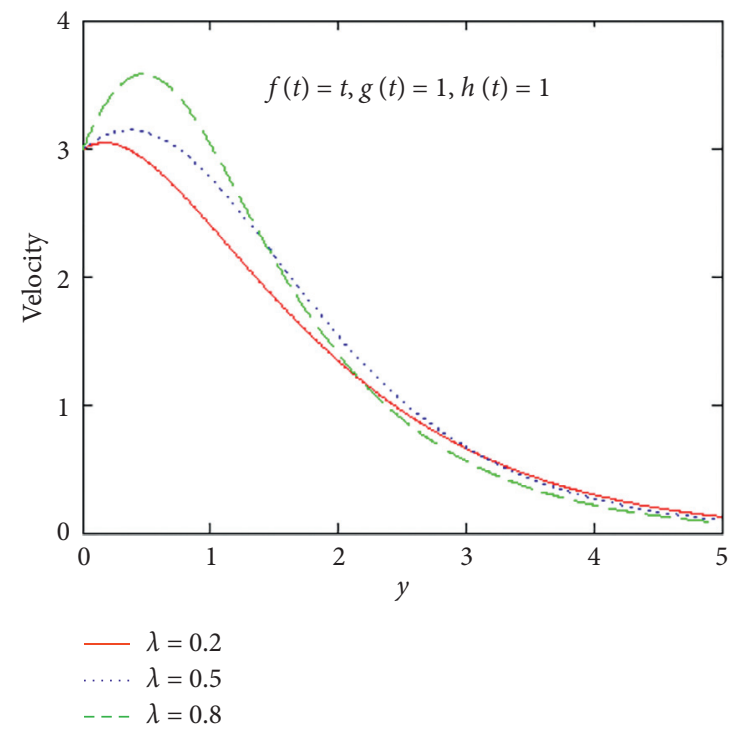

(b)

Figure 15: Continued. 


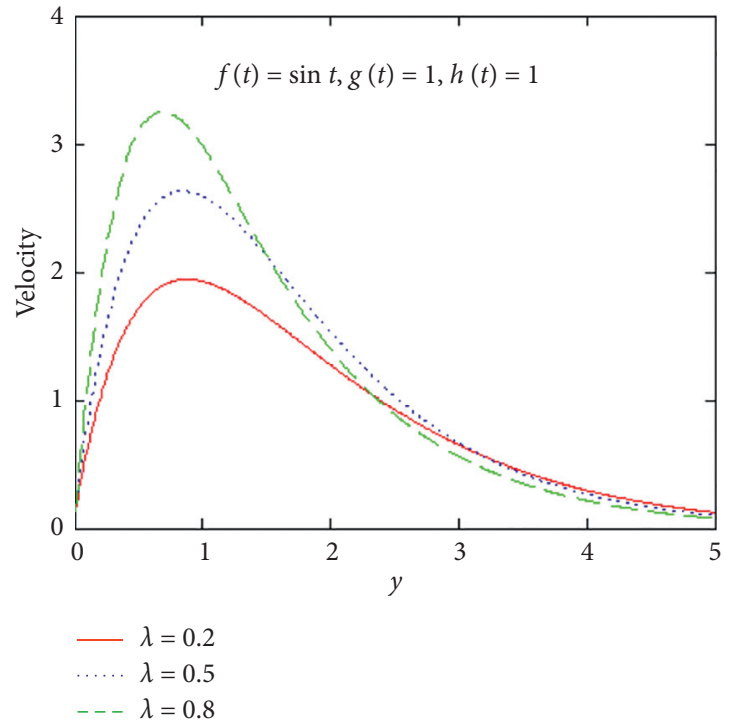

(c)

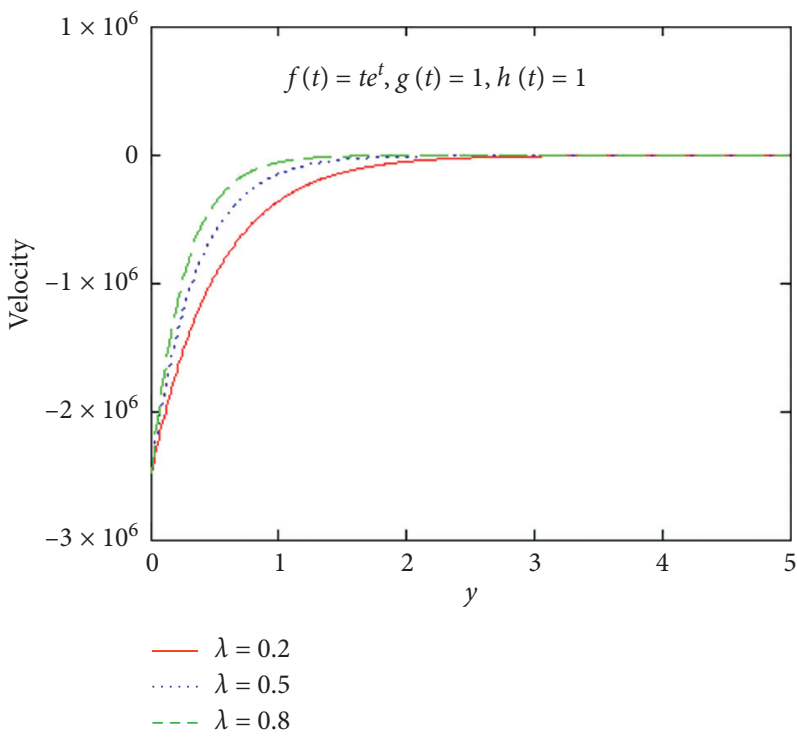

(e)

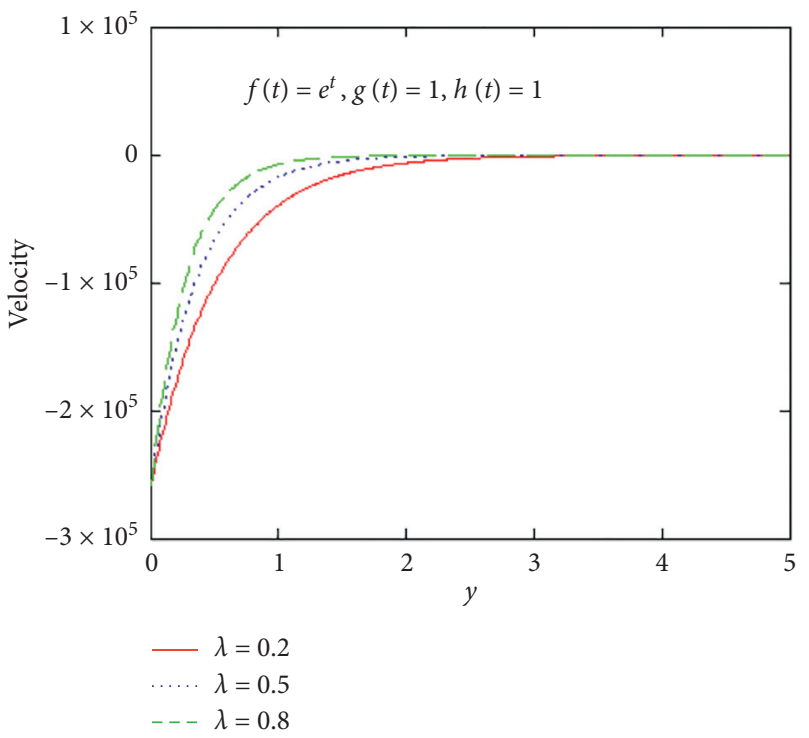

(d)

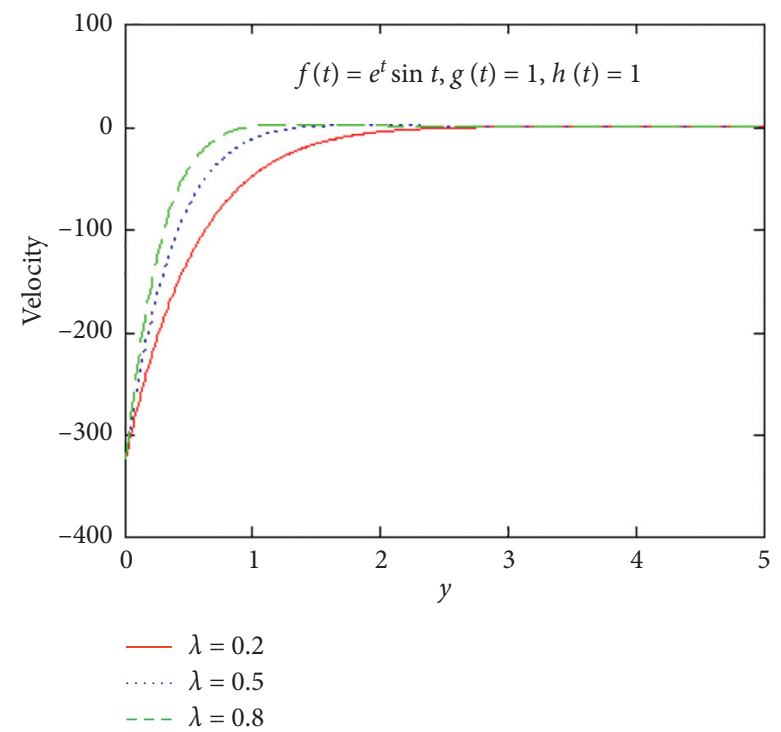

(f)

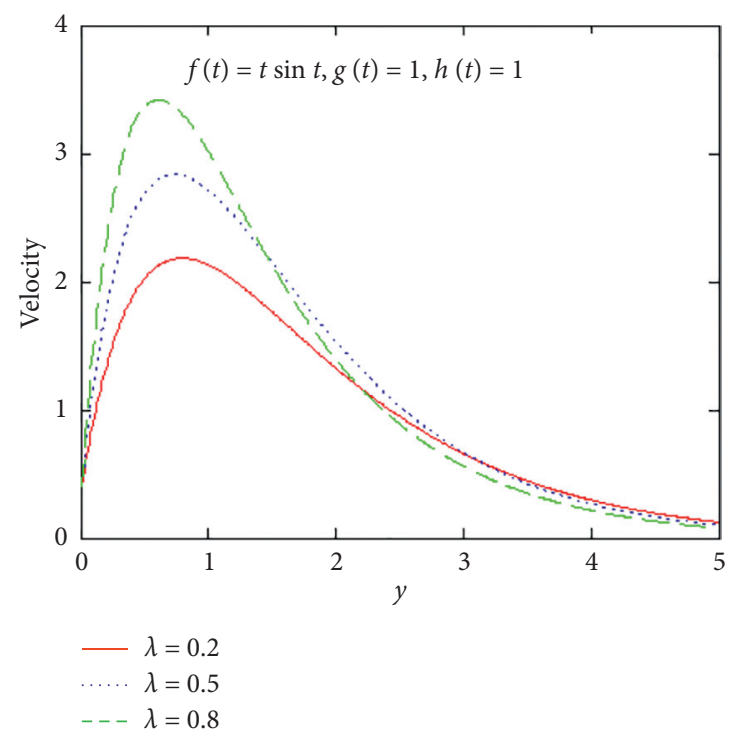

(g)

FIGURE 15: Profile of velocity for different values of $\lambda$ and $M=2.0, G_{C}=2.0, S=1.5, K_{c}=0.5, S_{c}=0.60, G_{T}=10.0, P_{r}=0.5$. 


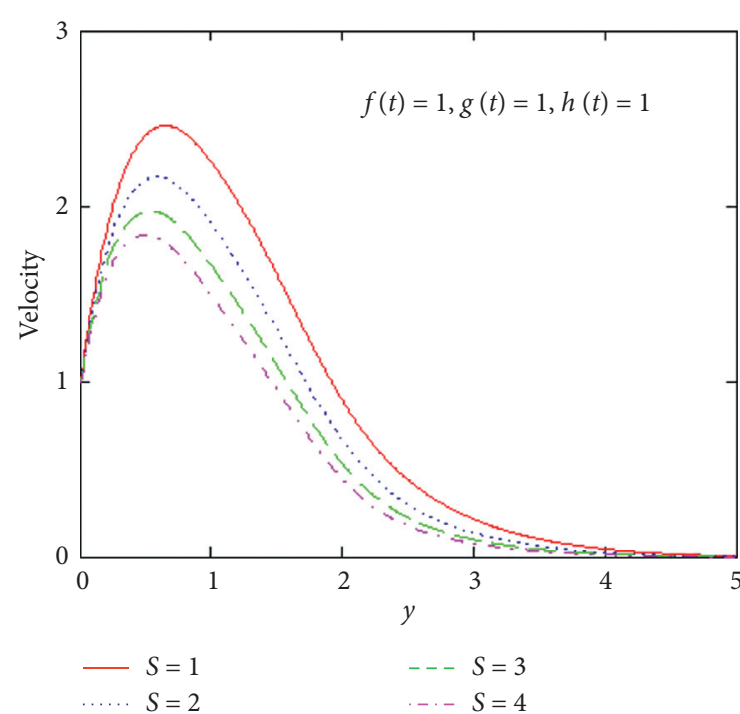

(a)

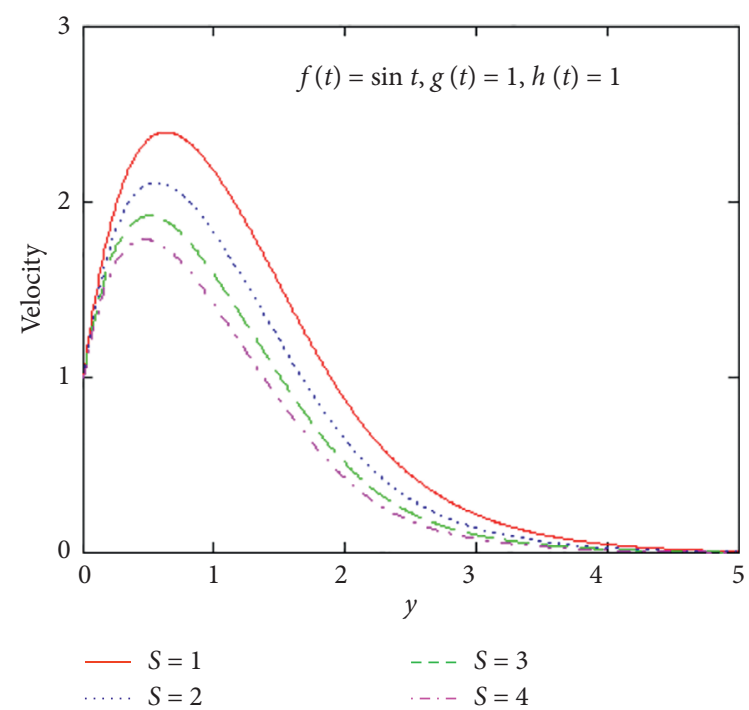

(c)

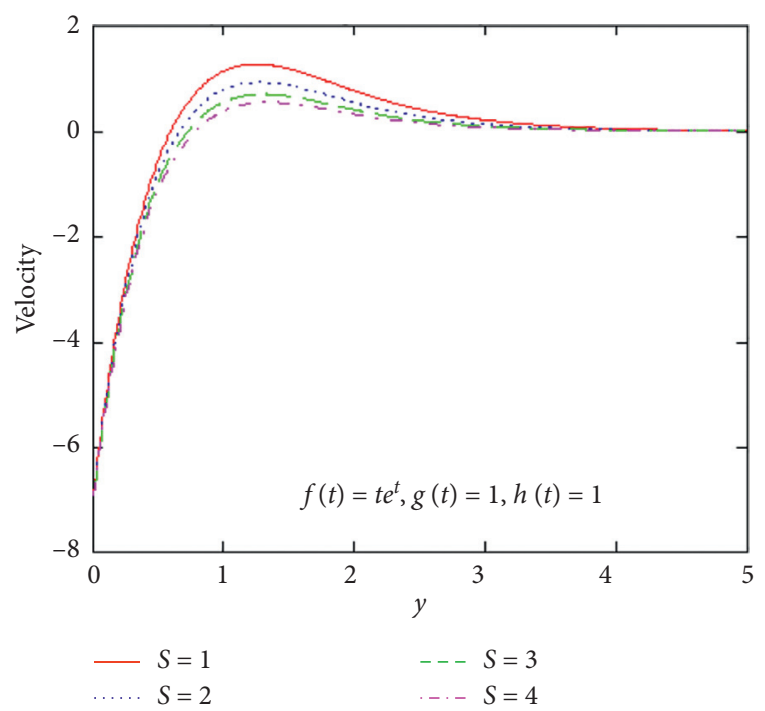

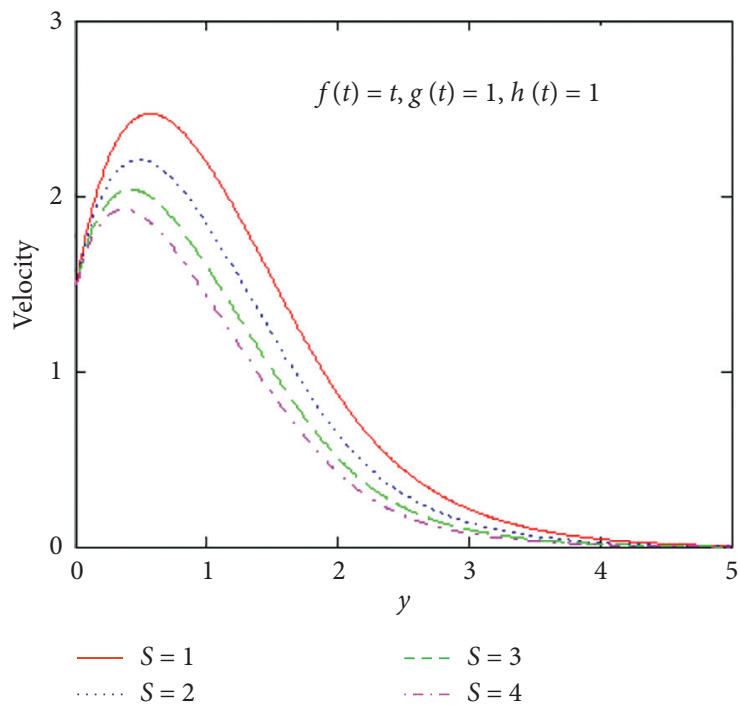

(b)

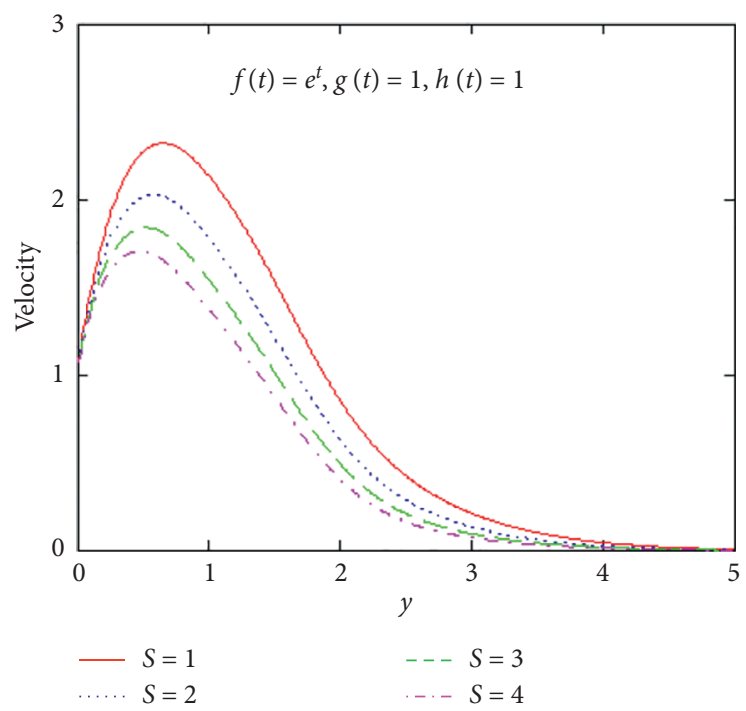

(d)

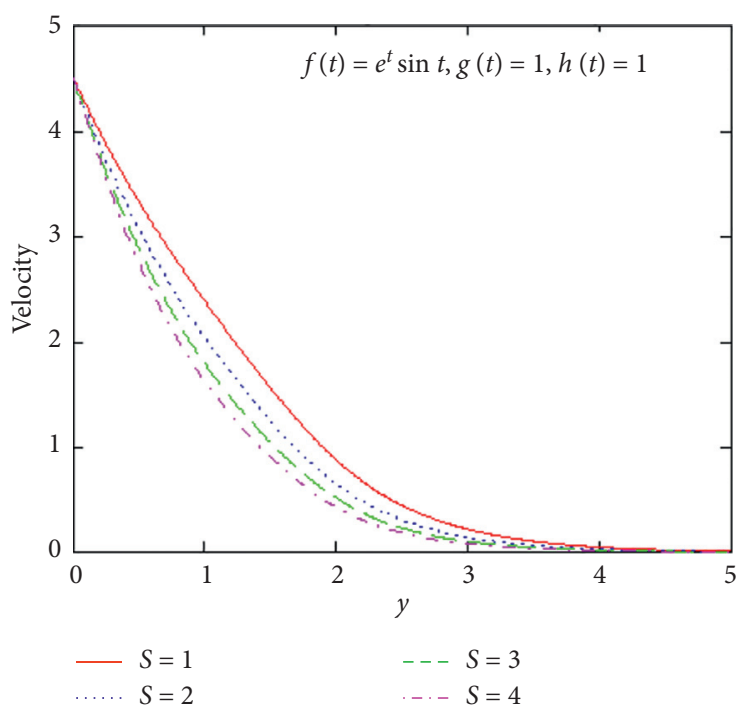

(f)

FIgURE 16: Continued. 


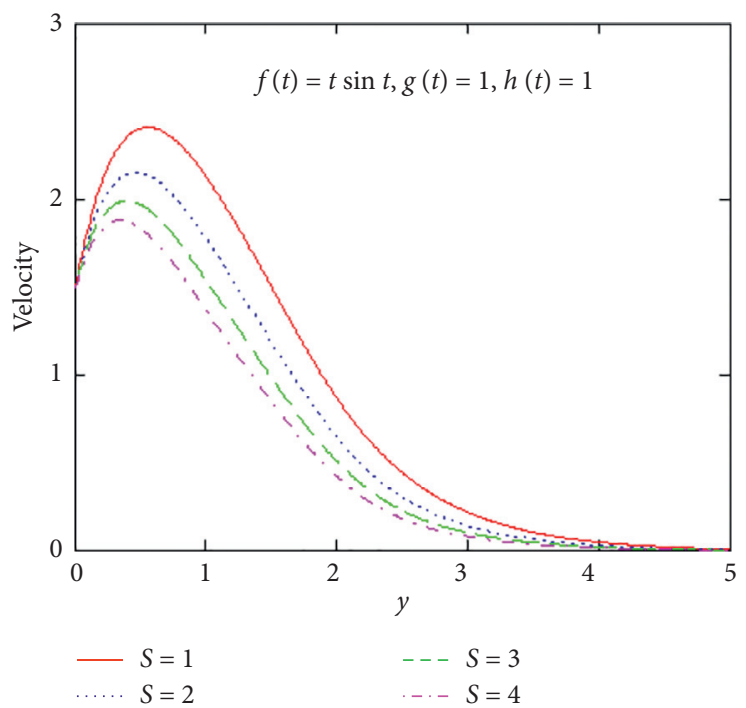

(g)

Figure 16: Profile of velocity for different values of $S$ and $M=2.0, G_{C}=2.0, \lambda=0.6, K_{c}=0.5, S_{c}=0.60, G_{T}=10.0, P_{r}=0.5$.

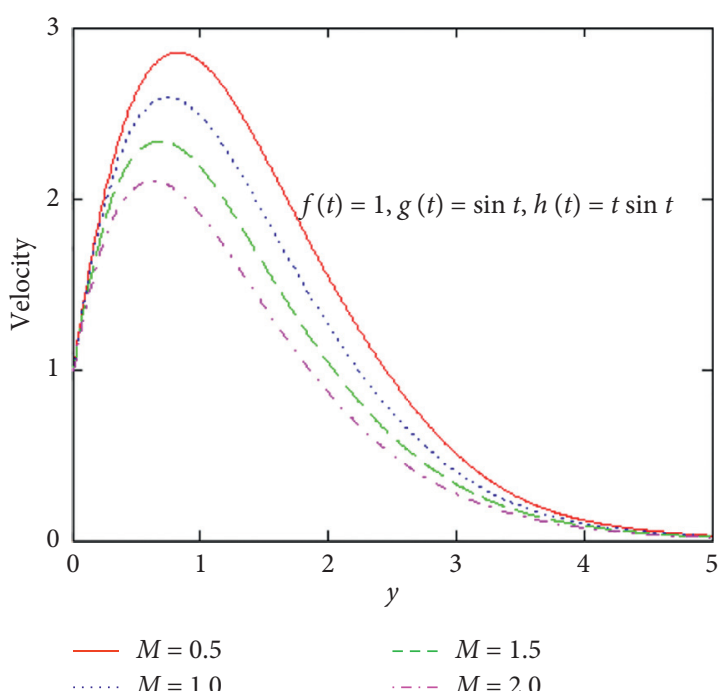

(a)

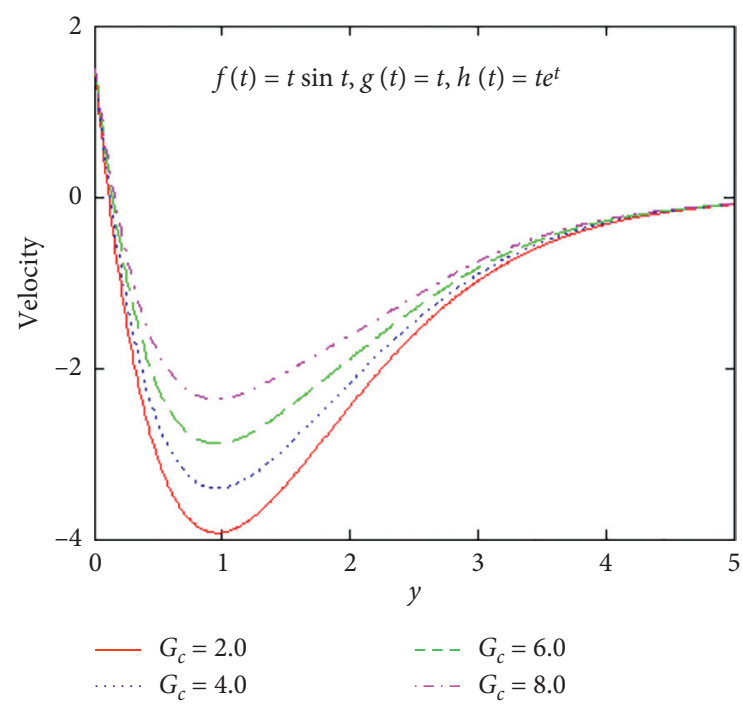

(c)

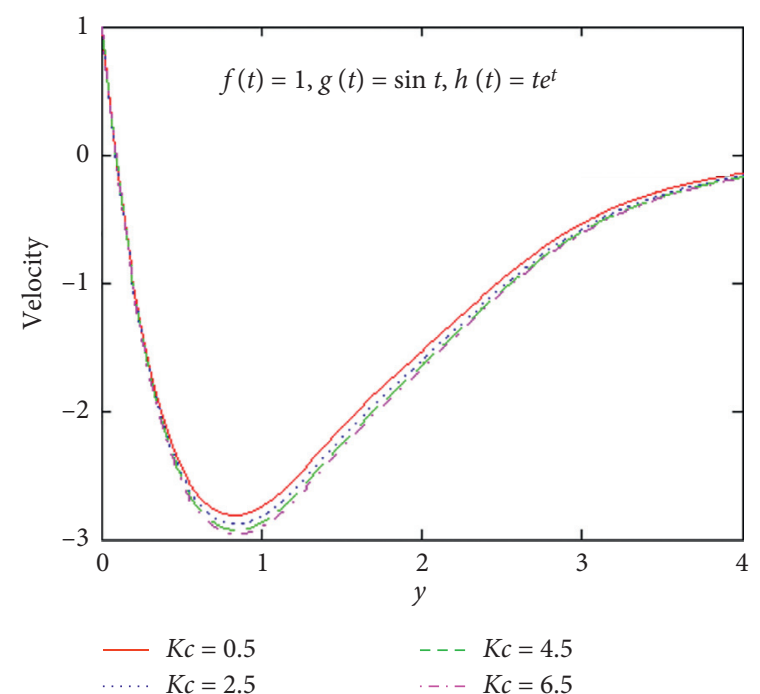

(b)

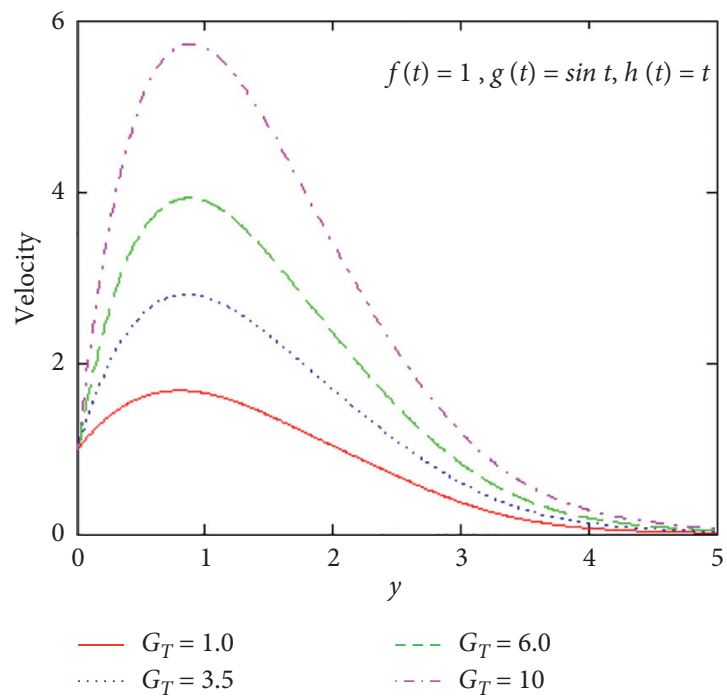

(d)

FIgURE 17: Continued. 


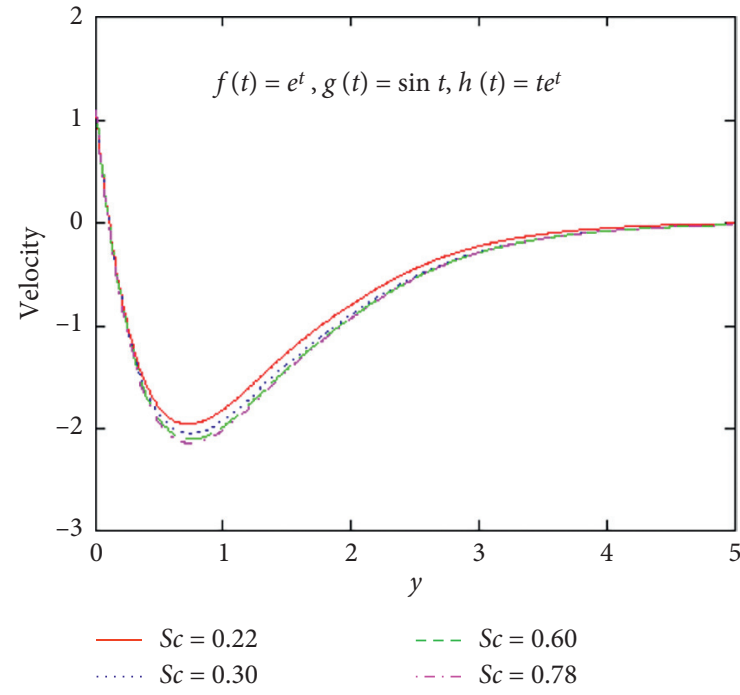

(e)

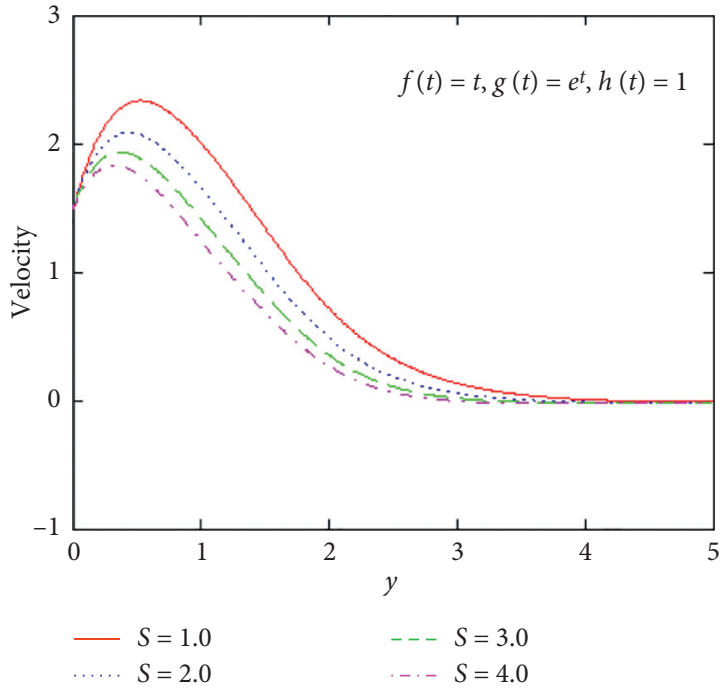

(f)

Figure 17: Profile of velocity for different values of $M, S, S_{c}, K_{c}, G_{T}, G_{C}$ and different choice of function for $f(t), g(t), h(t)$.

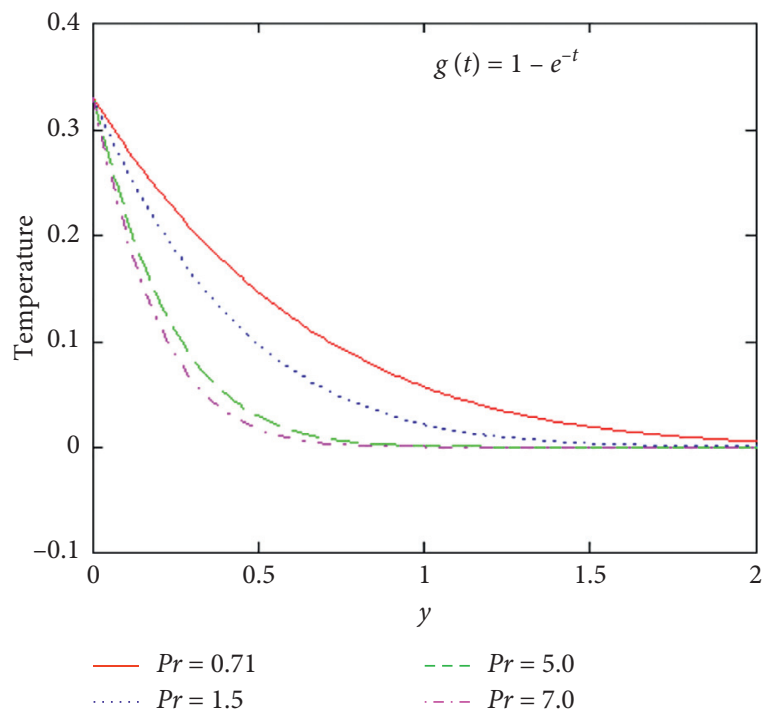

(a)

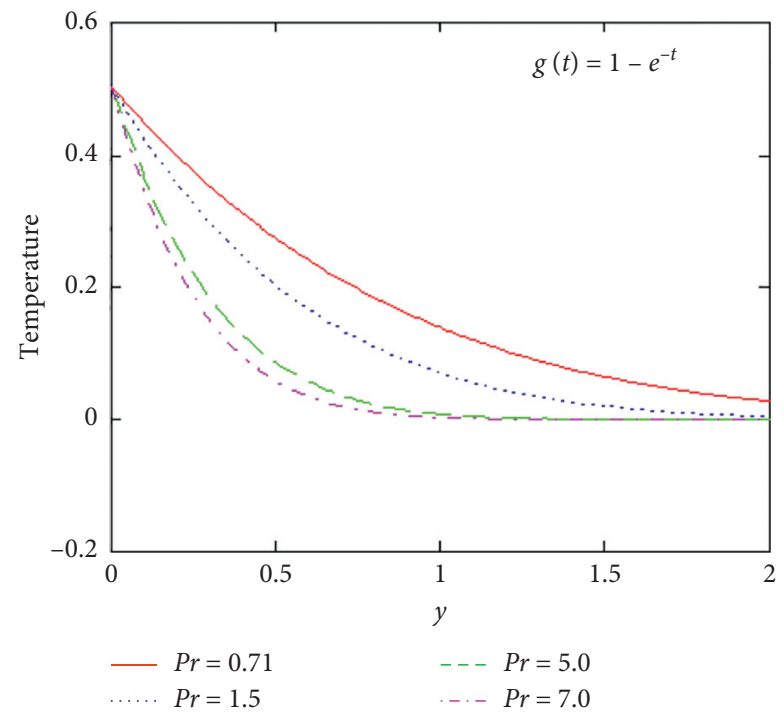

(b)

Figure 18: Temperature profile for different values of $P_{r}$ with $S=0.5$ and $g(t)=1-e^{-t}$. 


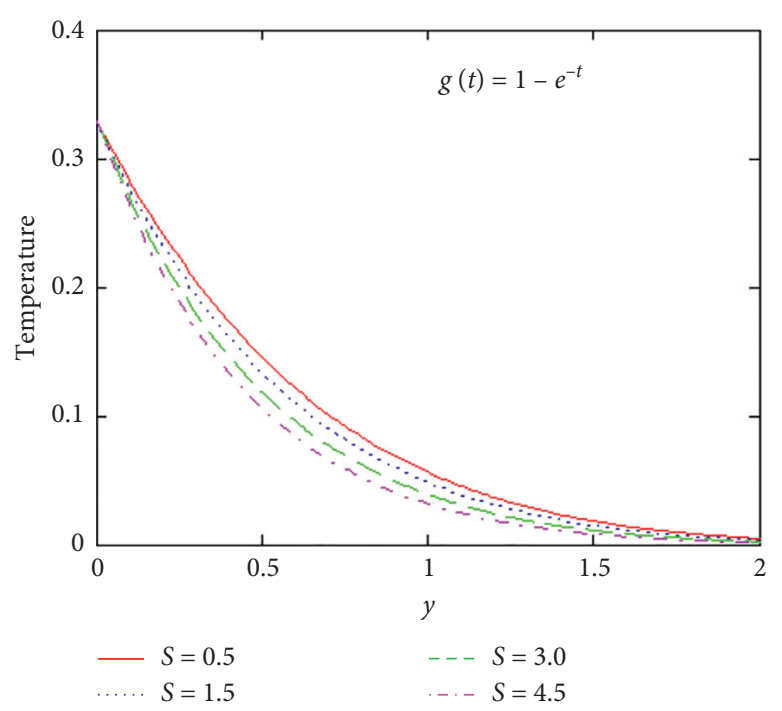

(a)

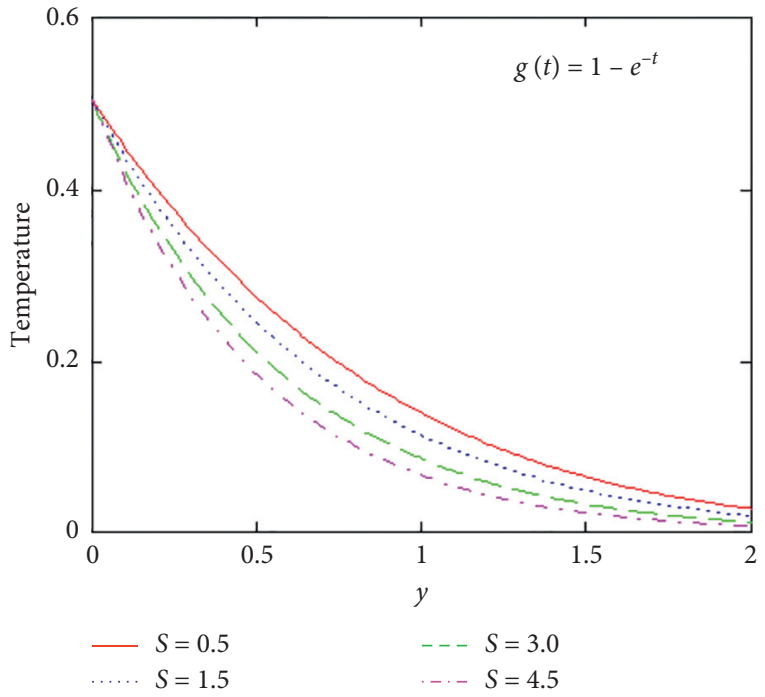

(b)

Figure 19: Temperature profile for different values of $S$ with $P_{r}=0.71$ and $g(t)=1-e^{-t}$.

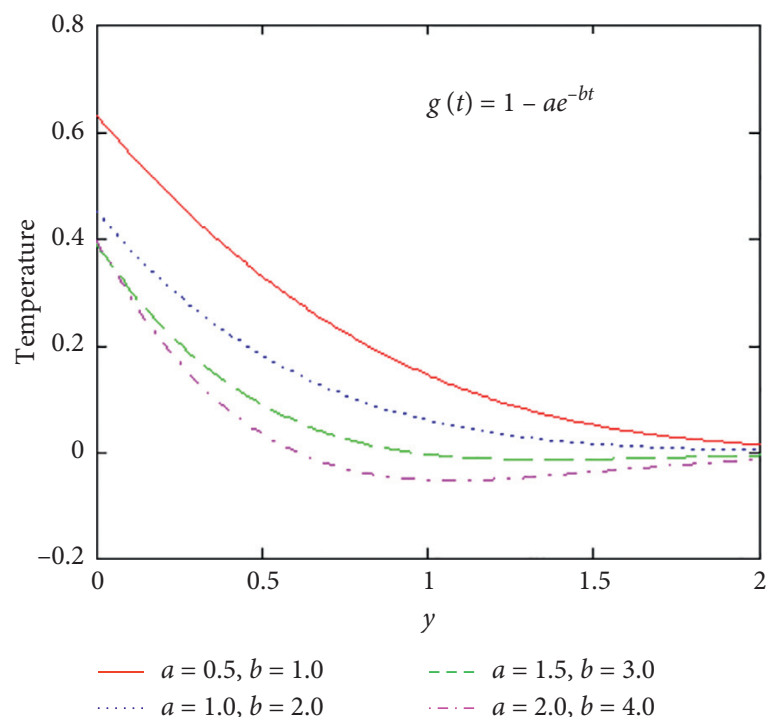

(a)

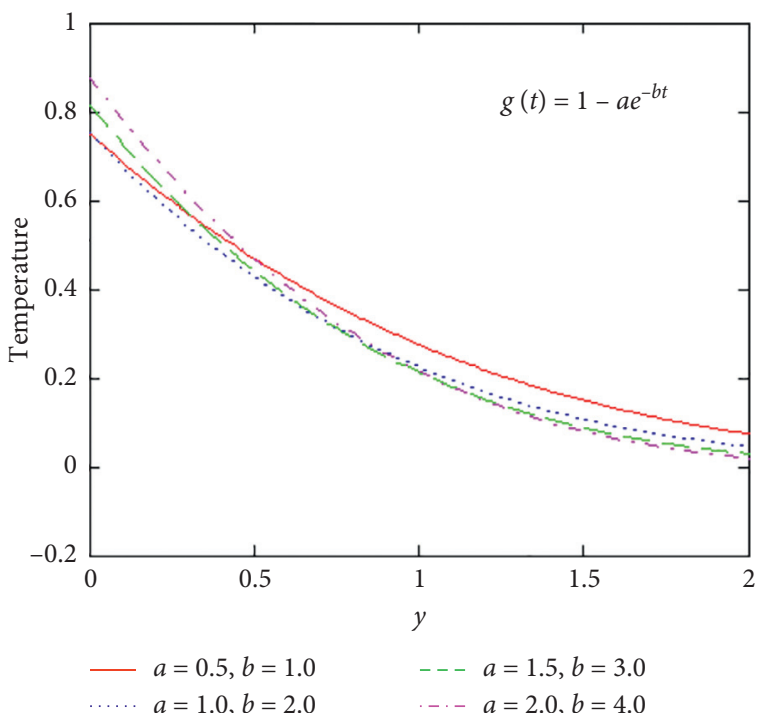

(b)

Figure 20: Temperature profile for different values of $a, b$ with $P_{r}=0.71, S=0.5$ and $g(t)=1-a e^{-b t}$.

\section{Conclusions}

A thorough investigation of MHD Maxwell fluid motion has been studied here under the effects of different parameters. The exact solutions are obtained for concentration, temperature, and velocity which satisfied the described initial and boundary conditions. Laplace transform is employed to obtain the exact solution and the behavior of different parameters on the flow of fluid along with different boundary conditions is investigated. Effects of chemical reaction coefficient, Schmidt number, and different boundary conditions on concentration, effects of Prandtl number, heat source, Newtonian heating, etc. on temperature, Magnetic parameter, Schmidt number, thermal Grashof number, relaxation parameter, mass Grashof number, Prandtl number, heat source, and chemical reaction impacts on the fluid motion are discussed. The results obtained are as follows:

(1) The boundary layer thickness of concentration decreases with the increase in the mass diffusivity $S_{c}$ and chemical reaction parameter $K_{C}$.

(2) The thermal boundary layer decreases with the increase in momentum boundary layer due to $P_{r}$ and heat absorption $S$. 
(3) Lorentz force effects due to $M$, momentum boundary layer effects due to $P_{r}$, mass diffusivity effects due to $S_{c}$, chemical reaction $K_{c}$, and heat absorption decrease the velocity with the increase of these parameters.

(4) Increase in buoyancy forces due to $G_{T}$ and $G_{C}$ stimulates the speed of fluid flow.
(5) A rise in relaxation time $\lambda$ reduces the fluid viscosity and results in acceleration of fluid flow.

\section{Appendix}

$$
\begin{aligned}
& B_{1}(y, t)= \begin{cases}0, & 0<t<y \lambda, \\
e^{-\alpha_{1} t} I_{0}\left(i \alpha_{3} \sqrt{t^{2}-\lambda y^{2}}\right), & t>y \lambda,\end{cases} \\
& B_{2}^{I I}(t)=\left(e^{-\alpha_{1} t}\left(\left(i \alpha_{3}\right) I_{1}\left(i \alpha_{3} t\right)+\delta(t)\right)\right)^{*}\left(1+\alpha_{1} t\right)+\left(\alpha_{3}^{2} e^{-\alpha_{1} t} I_{0}\left(i \alpha_{3} t\right)\right)^{*} t, \\
& B_{3}^{I I}(t)=(1+\lambda H(t))^{*}\left(A^{*}+B^{*} e^{-\left(\alpha_{4}+\alpha_{6}\right) t}+C^{*} e^{-\left(\alpha_{4}-\alpha_{6}\right) t}\right)^{*} e^{-\alpha_{1} t} I_{0}\left(i \alpha_{3} t\right), \\
& B_{4}^{I I}(t)=\left(1+\lambda H(t)^{*}\left(D^{*}+E^{*} e^{-\left(\alpha_{7}+\alpha_{9}\right) t}+F^{*} e^{-\left(\alpha_{7}-\alpha_{9}\right) t}\right){ }^{*} e^{-\alpha_{1} t} I_{0}\left(i \alpha_{3} t\right),\right. \\
& B_{2}^{I I I}(t)=\left(e^{-\alpha_{1} t}\left(\left(i \alpha_{3}\right) I_{1}\left(i \alpha_{3} t\right)+\delta(t)\right)\right)^{*}\left(\cos t+\alpha_{1} \sin t\right)+\left(\alpha_{3}^{2} e^{-\alpha_{1} t} I_{0}\left(i \alpha_{3} t\right)\right)^{*} \sin t, \\
& B_{3}^{I I I}(t)=\left(\cos t+\lambda H(t)^{*} \cos t\right)^{*}\left(A^{*}+B^{*} e^{-\left(\alpha_{4}+\alpha_{6}\right) t}+C^{*} e^{-\left(\alpha_{4}-\alpha_{6}\right) t}\right)^{*} e^{-\alpha_{1} t} I_{0}\left(i \alpha_{3} t\right), \\
& B_{4}^{I I I}(t)=\left(\cos t+\lambda H(t)^{*} \cos t\right)^{*}\left(D^{*}+E^{*} e^{-\left(\alpha_{7}+\alpha_{9}\right) t}+F^{*} e^{-\left(\alpha_{7}-\alpha_{9}\right) t}\right)^{*} e^{-\alpha_{1} t} I_{0}\left(i \alpha_{3} t\right), \\
& B_{2}^{I V}(t)=\left(e^{-\alpha_{1} t}\left(\left(i \alpha_{3}\right) I_{1}\left(i \alpha_{3} t\right)+\delta(t)\right)\right)^{*}\left(\left(H(t)+\alpha_{1}\right)^{*} e^{t}\right)+\left(\alpha_{3}^{2} e^{-\alpha_{1} t} I_{0}\left(i \alpha_{3} t\right)\right)^{*} e^{t}, \\
& B_{3}^{I V}(t)=\left(e^{t}+\lambda H(t)^{*} e^{t}\right)^{*}\left(A^{*}+B^{*} e^{-\left(\alpha_{4}+\alpha_{6}\right) t}+C^{*} e^{-\left(\alpha_{4}-\alpha_{6}\right) t}\right)^{*} e^{-\alpha_{1} t} I_{0}\left(i \alpha_{3} t\right), \\
& B_{4}^{I V}(t)=\left(e^{t}+\lambda H(t)^{*} e^{t}\right)^{*}\left(D^{*}+E^{*} e^{-\left(\alpha_{7}+\alpha_{9}\right) t}+F^{*} e^{-\left(\alpha_{7}-\alpha_{9}\right) t}\right)^{*} e^{-\alpha_{1} t} I_{0}\left(i \alpha_{3} t\right), \\
& B_{2}^{V}(t)=\left(e^{-\alpha_{1} t}\left(\left(i \alpha_{3}\right) I_{1}\left(i \alpha_{3} t\right)+\delta(t)\right)\right)^{*}\left(e^{t}+\left(1+\alpha_{1}\right) t e^{t}\right)+\left(\alpha_{3}^{2} e^{-\alpha_{1} t} I_{0}\left(i \alpha_{3} t\right)\right)^{*} t e^{t}, \\
& B_{3}^{V}(t)=\left(e^{t}(1+t)+\lambda H(t)^{*} e^{t}(1+t)\right)^{*}\left(A^{*}+B^{*} e^{-\left(\alpha_{4}+\alpha_{6}\right) t}+C^{*} e^{-\left(\alpha_{4}-\alpha_{6}\right) t}\right) \\
& { }^{*} e^{-\alpha_{1} t} I_{0}\left(i \alpha_{3} t\right), \\
& B_{4}^{V}(t)=\left(e^{t}(1+t)+\lambda H(t)^{*} e^{t}(1+t)\right)^{*}\left(D^{*}+E^{*} e^{-\left(\alpha_{7}+\alpha_{9}\right) t}+F^{*} e^{-\left(\alpha_{7}-\alpha_{9}\right) t}\right) \\
& { }^{*} e^{-\alpha_{1} t} I_{0}\left(i \alpha_{3} t\right) \text {. } \\
& B_{2}^{V I}(t)=\left(e^{-\alpha_{1} t}\left(\left(i \alpha_{3}\right) I_{1}\left(i \alpha_{3} t\right)+\delta(t)\right)\right)^{*}\left(e^{t} \cos t+\left(1+\alpha_{1}\right) e^{t} \sin t\right)+\left(\alpha_{3}^{2} e^{-\alpha_{1} t} I_{0}\left(i \alpha_{3} t\right)\right) \\
& { }^{*} e^{t} \sin t \text {. } \\
& B_{3}^{V I}(t)=\left(e^{t} \cos t+e^{t} \sin t+\lambda H(t)^{*}\left(e^{t} \cos t+e^{t} \sin t\right)\right)^{*}\left(A^{*}+B^{*} e^{-\left(\alpha_{4}+\alpha_{6}\right) t}+C^{*} e^{-\left(\alpha_{4}-\alpha_{6}\right) t}\right) \\
& { }^{*} e^{-\alpha_{1} t} I_{0}\left(i \alpha_{3} t\right) \text {. }
\end{aligned}
$$




$$
\begin{aligned}
& B_{4}^{V I}(t)=\left(e^{t} \cos t+e^{t} \sin t+\lambda H(t)^{*}\left(e^{t} \cos t+e^{t} \sin t\right)\right)^{*}\left(D^{*}+E^{*} e^{-\left(\alpha_{7}+\alpha_{9}\right) t}+F^{*} e^{-\left(\alpha_{7}-\alpha_{9}\right) t}\right) \\
& e^{* \alpha_{1} t} I_{0}\left(i \alpha_{3} t\right) \\
& B_{2}^{V I I}(t)=\left(e^{-\alpha_{1} t}\left(\left(i \alpha_{3}\right) I_{1}\left(i \alpha_{3} t\right)+\delta(t)\right)\right)^{*}\left(H(t)+\left(1+\alpha_{1}\right) t \sin t\right)+\left(\alpha_{3}^{2} e^{-\alpha_{1} t} I_{0}\left(i \alpha_{3} t\right)\right) \\
& { }^{*} t \sin t \\
& B_{3}^{V I I}(t)=\left((t \sin t+t \cos t)+\lambda H(t)^{*}(\sin t+\lambda \cos t)\right)^{*}\left(A^{*}+B^{*} e^{-\left(\alpha_{4}+\alpha_{6}\right) t}+C^{*} e^{-\left(\alpha_{4}-\alpha_{6}\right) t}\right) \\
& e^{-\alpha_{1} t} I_{0}\left(i \alpha_{3} t\right) \\
& B_{4}^{V I I}(t)=\left((t \sin t+t \cos t)+\lambda H(t)^{*}(\sin t+\lambda \cos t)\right)^{*}\left(D^{*}+E^{*} e^{-\left(\alpha_{7}+\alpha_{9}\right) t}+F^{*} e^{-\left(\alpha_{7}-\alpha_{9}\right) t}\right) \\
& { }^{*} e^{-\alpha_{1} t} I_{0}\left(i \alpha_{3} t\right) \\
& L^{-1}\left[\frac{e^{-b \sqrt{q^{2}-a^{2}}}}{\sqrt{q^{2}-a^{2}}}\right]= \begin{cases}0, & 0<t<b, b>0, \\
I_{0}\left(a \sqrt{t^{2}-b^{2}}\right), & t>b, \operatorname{Re}(q)>\operatorname{Re}(a),\end{cases} \\
& L^{-1}[F(q+m)]=f(t) e^{-m t} \\
& L^{-1}\left[\frac{\exp (-y \sqrt{q+a})}{q-b}\right]=e^{b t} \Phi(y ; t, a+b), \\
& \Phi(y, t ; a)=\frac{1}{2}\left\{e^{y \sqrt{a}} \operatorname{ercf}\left(\frac{y}{2 \sqrt{t}}+\sqrt{a t}\right)+e^{-y \sqrt{a}} \operatorname{ercf}\left(\frac{y}{2 \sqrt{t}}-\sqrt{a t}\right)\right\}, \\
& L^{-1}\left[\frac{\exp (-y \sqrt{q+a})}{q-b}\right]=e^{b t} \Psi(y ; t, a+b), \\
& \Psi(y, t ; a)=\frac{1}{2}\left\{e^{y \sqrt{a}} \operatorname{ercf}\left(\frac{y}{2 \sqrt{t}}+\sqrt{a t}\right)+e^{-y \sqrt{a}} \operatorname{ercf}\left(\frac{y}{2 \sqrt{t}}-\sqrt{a t}\right)\right\} .
\end{aligned}
$$

\section{Data Availability}

The data used to support the findings of this study are included within the article.

\section{Conflicts of Interest}

The authors declare that they have no conflicts of interest.

\section{Acknowledgments}

The authors are thankful and grateful to their respective departments and universities for supporting and facilitating the research work.

\section{References}

[1] C.-Y. Cheng, "Natural convection heat and mass transfer from a sphere in micropolar fluids with constant wall temperature and concentration," International Communications in Heat and Mass Transfer, vol. 35, no. 6, pp. 750-755, 2008.
[2] K. R. Cramer and S. I. Pai, Magneto Fluid Dynamics for Engineers and Applied Physicists, pp. 204-237, McGraw-Hill Book Co New York, New York, NY, USA, 1973.

[3] N. F. M. Noor, S. Abbasbandy, and I. Hashim, "Heat and mass transfer of thermophoretic MHD flow over an inclined radiate isothermal permeable surface in the presence of heat source/ sink," International Journal of Heat and Mass Transfer, vol. 55, no. 7-8, pp. 2122-2128, 2012.

[4] S. M. Mousazadeh, M. M. Shahmardan, T. Tavangar, K. Hosseinzadeh, and D. D. Ganji, "Numerical investigation on convective heat transfer over two heated wall-mounted cubes in tandem and staggered arrangement," Theoretical and Applied Mechanics Letters, vol. 8, no. 3, pp. 171-183, 2018.

[5] F. M. Oudina, F. Redouane, F. Redouane, and C. Rajashekhar, "Convection heat transfer of $\mathrm{MgO}-\mathrm{Ag} /$ water magneto-hybrid nanoliquid flow into a special porous enclosure," Algerian Journal of Renewable Energy and Sustainable Development, vol. 2, no. 2, pp. 84-95, 2020.

[6] S. S. Das, A. Satapathy, J. K. Das, and J. P. Panda, "Mass transfer effects on MHD flow and heat transfer past a vertical porous plate through a porous medium under oscillatory suction and heat source," International Journal of Heat and Mass Transfer, vol. 52, no. 25-26, pp. 5962-5969, 2009. 
[7] S. M. Abo-Dahab, M. A. Abdelhafez, F. Mebarek-Oudina, and S. M. Bilal, "MHD Casson nanofluid flow over nonlinearly heated porous medium in presence of extending surface effect with suction/injection," Indian Journal of Physics, vol. 23, 2021.

[8] S. Sajad, A. Nori, K. Hosseinzadeh, and D. D. Ganji, "Hydrothermal analysis of MHD squeezing mixture fluid suspended by hybrid nano particles between two parallel plates," Case Studies in Thermal Engineering, vol. 21, Article ID 100650, 2020

[9] N. Iftikhar, S. M. Husnine, and M. B. Riaz, "Heat and mass transfer in MHD Maxwell fluid over an infinite vertical plate," Journal of Prime Research in Mathematics, vol. 15, pp. 63-80, 2019.

[10] M. Ahmed, "Megahed, Variable fluid properties and variable heat flux effects on the flow and heat transfer in a nonNewtonian Maxwell fluid over an unsteady stretching sheet with slip velocity," Chinese Physics B, vol. 922, Article ID 094701, 2013.

[11] S. Marzougui, M. Bouabid, F. Mebarek-Oudina, N. AbuHamdeh, M. Magherbi, and K. Ramesh, "A computational analysis of heat transport irreversibility phenomenon in a magnetized porous channel," International Journal of $\mathrm{Nu}$ merical Methods for Heat \& Fluid Flow, vol. 43, 2020.

[12] D. Belatrache, N. Saifi, A. Harrouz, and S. Bentouba, "Modelling and numerical investigation of the thermal properties effect on the soil temperature in Adrar region," Algerian Journal of Renewable Energy and Sustainable Development, vol. 2, no. 2, pp. 165-174, 2020.

[13] K. Hosseinzadeh, A. R. Mogharrebi, A. Asadi, M. Paikar, and D. D. Ganji, "Effect of fin and hybrid nano-particles on solid process in hexagonal triplex latent heat thermal energy storage system," Journal of Molecular Liquids, vol. 300, Article ID 112347, 2020.

[14] M. Gholinia, S. Gholinia, K. Hosseinzadeh, and D. D. Ganji, "Investigation on ethylene glycol nano fluid flow over a vertical permeable circular cylinder under effect of magnetic field," Results in Physics, vol. 9, pp. 1525-1533, 2018.

[15] J. Rahimi, D. D. Ganji, M. Khaki, and K. Hosseinzadeh, "Solution of the boundary layer flow of an Eyring-Powell nonNewtonian fluid over a linear stretching sheet by collocation method," Alexandria Engineering Journal, vol. 56, no. 4, pp. 621-627, 2017.

[16] K. Hosseinzadeh, M. A. E. Moghaddam, A. Asadi, A. R. Mogharrebi, and D. D. Ganji, "Effect of internal fins along with Hybrid Nano-Particles on solid process in star shape triplex Latent Heat Thermal Energy Storage System by numerical simulation," Renewable Energy, vol. 154, pp. 497507, 2020.

[17] U. S. Rajput and S. Kumar, "Radiation effects on MHD flow past an impulsively started vertical plate with variable heat and mass transfer," IJAMM, International Journal of Applied Mathematics and Mechanics, vol. 8, pp. 66-85, 2012.

[18] A. S. Gupta, "Steady and transient free convection of an electrically conducting fluid from a vertical plate in the presence of magnetic field," Archives of Applied Science Research, vol. 8, pp. 319-333, 1961.

[19] M. F. El-Amin, "MHD free convection and mass transfer flow in micropolar fluid with constant suction," Journal of Magnetism and Magnetic Materials, vol. 243, no. 3, pp. 567-574, 2006.

[20] S. Nadeem, R. U. Haq, and Z. H. Khan, "Numerical study of MHD boundary layer flow of a Maxwell fluid past a stretching sheet in the presence of nanoparticles," Journal of the Taiwan
Institute of Chemical Engineers, vol. 45, no. 1, pp. 121-126, 2014.

[21] J. Zhao, L. Zheng, X. Zhang, and F. Liu, "Unsteady natural convection boundary layer heat transfer of fractional Maxwell viscoelastic fluid over a vertical plate," International Journal of Heat and Mass Transfer, vol. 97, pp. 760-766, 2016.

[22] N. Ahmad, "Soret and radiation effects on transient MHD free convection from an impulsively started infinite verticl plate," The Journal of Heat Transfer, vol. 134, Article ID 062701, 2012.

[23] R. C. Chaudhary and A. Jain, "An exact solution of magnetohydrodynamic convection flow past an accelerated surface embedded in a porous medium," International Journal of Heat and Mass Transfer, vol. 53, no. 7-8, pp. 1609-1611, 2010.

[24] K. Das, "Exacat solution of MHD free convection flow and mass transfer near a moving vertical plate in presesnce of thermal radiation," African Journal of Mathematical Physics, vol. 8, pp. 29-41, 2010.

[25] K. Das and S. Jana, "Heat and mass transfer effects on unsteady MHD free convection flow near a moving plate in porous medium," Bulletin of Society of Mathematicians, vol. 17, pp. 15-32, 2010.

[26] C. Fetecau and N. A. Shah, "Dumitru vieru general solutions for hydromagnetic free convection flow over an infinite plate with Newtonian heating, mass diffusion and chemical reaction," Communications in Theoretical Physics, vol. 68, p. 6, 2017.

[27] N. A. Shah, "Heat and mass transfer in hydromagnetic flows of viscous fluids over a flat plate" Phd thesis, Government College University Lahore, Lahore, Pakistan, 2019. 